\title{
Abelian Higgs model in power-law inflation: the propagators in the unitary gauge
}

\author{
Dražen Glavan, ${ }^{a, b}$ Anja Marunović, ${ }^{c}$ Tomislav Prokopec ${ }^{d}$ and Zahra Zahraee ${ }^{e}$ \\ ${ }^{a}$ Centre for Cosmology, Particle Physics and Phenomenology (CP3), \\ Université catholique de Louvain, \\ Chemin du Cyclotron 2, 1348 Louvain-la-Neuve, Belgium \\ ${ }^{b}$ Division of Theoretical Physics, Rudjer Bošković Institute, \\ Bijenička cesta 54, HR-10000 Zagreb, Croatia \\ ${ }^{c}$ Amsterdam, The Netherlands \\ ${ }^{d}$ Institute for Theoretical Physics (ITF), Spinoza Institute \& EMMEФ, \\ Faculty of Science, Utrecht University, \\ Postbus 80195, 3508 TD Utrecht, The Netherlands \\ ${ }^{e}$ Department of Physics, McGill University, \\ 3600 Rue University, Montréal, QC H3A 2T8, Canada \\ E-mail: drazen.glavan@uclouvain.be, anja.marun@gmail.com, \\ t.prokopec@uu.nl, zr.zahraee@physics.mcgill.ca
}

ABSTRACT: We consider the Abelian Higgs model in the broken phase as a spectator in cosmological spaces of general $D$ space-time dimensions, and allow for the condensate to be time-dependent. We fix the unitary gauge using Dirac's formalism for constrained systems, and then quantize the gauge-fixed system. Vector and scalar perturbations develop timedependent masses. We work out their propagators assuming the cosmological background is that of power-law inflation, characterized by a constant principal slow-roll parameter, and that the scalar condensate is in the attractor regime, scaling as the Hubble rate. Our propagators correctly reduce to known results in the Minkowski and de Sitter space limits. We use the vector propagator to compute the equal-time correlators of electric and magnetic fields and find that at super-Hubble separations the former is enhanced, while the latter is suppressed compared to the vacuum fluctuations of the massless vector field. These correlators satisfy the hierarchy governed by Faraday's law.

Keywords: Cosmology of Theories beyond the SM, Spontaneous Symmetry Breaking, Gauge Symmetry, Higgs Physics

ArXiv ePrint: 2005.05435 


\section{Contents}

1 Introduction $\quad 2$

2 FLRW and power-law inflation $\quad 9$

3 Abelian Higgs model in cosmological spaces $\quad 12$

$\begin{array}{ll}3.1 \text { Hamiltonian formulation and gauge-fixing } & 13\end{array}$

$\begin{array}{lll}3.2 & \text { Perturbations } & 17\end{array}$

$\begin{array}{lll}3.3 & \text { Quantization } & 19\end{array}$

4 Condensate dynamics $\quad 19$

$\begin{array}{llr}5 & \text { Scalar perturbations } & 20\end{array}$

6 Dynamics of vector perturbations $\quad 21$

6.1 Scalar-vector decomposition 22

6.2 Fourier decomposition 24

6.3 Dynamics of the transverse sector 25

6.4 Dynamics of the longitudinal sector 26

$\begin{array}{lll}7 & \text { Vector field two-point functions } & 27\end{array}$

$\begin{array}{lll}7.1 & \text { Equations of motion for two-point functions } & 27\end{array}$

$\begin{array}{ll}7.2 \text { Choice of the state } & 30\end{array}$

$\begin{array}{lll}7.3 & \text { Two-point functions as mode sums } & 31\end{array}$

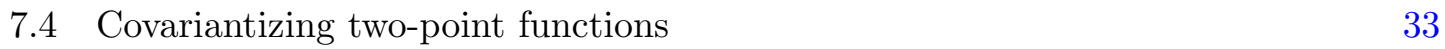

7.5 Various limits 36

$\begin{array}{lll}\text { 7.5.1 De Sitter limit } & 37\end{array}$

$\begin{array}{lll}7.5 .2 & \text { Flat space limit } & 37\end{array}$

$\begin{array}{lll}\text { 7.5.3 Coincidence limit } & 39\end{array}$

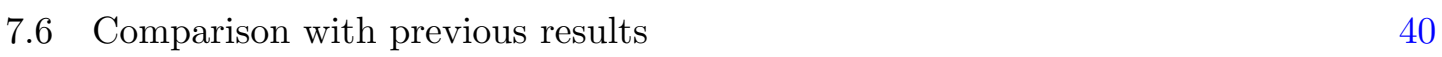

8 Field strength correlator $\quad 43$

8.1 Equal time E\&M correlators $\quad 45$

$\begin{array}{lll}\text { 8.1.1 Sub-Hubble limit } & 47\end{array}$

$\begin{array}{lll}\text { 8.1.2 Super-Hubble limit } & 47\end{array}$

9 Discussion $\quad 50$

A Scalar mode functions in power-law inflation $\quad 54$

$\begin{array}{ll}\text { B Rescaled propagator function } \mathcal{F}_{\nu} & 54\end{array}$

$\begin{array}{ll}\text { C Useful identity for } \mathcal{F}_{\nu} & 56\end{array}$

$\begin{array}{ll}\text { D Various derivative identities } & 57\end{array}$ 


\section{Introduction}

Quantum effects in primordial inflation are particularly strong for light fields nonconformally coupled to the expanding space-time. There are two such fields - the graviton and the light/massless scalar - that experience abundant gravitational particle production even at tree level [1-3]. This can lead to sizable quantum loop corrections to their evolution, or the evolution other fields they couple to. There exists a considerable body of literature on one-loop quantum effects in de Sitter space, see e.g. ref. [4] and references therein, that ascertains that, whenever loop corrections are important, they grow in time as the logarithm of the scale factor, $\sim \ln (a)$, as generally predicted by Weinberg's theorem $[5,6]$.

The primordial inflating Universe is often approximated by de Sitter space (more precisely the expanding Poincaré patch of de Sitter space) with a constant Hubble rate, $\dot{H}=0$. Even though this is true in many cases, the actual slow-roll inflation space-time deviated from the exact de Sitter. This deviation is conveniently parametrized by slow roll parameters, of which the most important is the principal slow-roll parameter $\epsilon=-\dot{H} / H^{2} \lesssim 0.01$. Taking account of these deviations is particularly important for the correct modeling of the scalar curvature perturbation. Furthermore, these deviations can be important when growing secular corrections induced by quantum loops are concerned. The logarithmic growth of de Sitter might turn into a power-law growth, schematically,

$$
\ln (a) \quad \longrightarrow \quad \frac{a^{\# \epsilon}-1}{\# \epsilon},
$$

where \# is a numerical factor. For $\ln (a) \lesssim 1 / \epsilon$ the two are virtually indistinguishable, as assumed by Weinberg's theorem. However, for longer inflation when $\ln (a) \gg 1 / \epsilon \sim 10^{2}$ the scaling becomes drastically different. It is unclear for how long inflation lasted, and it is imperative to investigate not only if there are large secular quantum effects in the primordial universe, but also how do they behave in more realistic inflationary spaces. This is particularly important in cases where \# in eq. (1.1) is a positive number, which can happen when light fields are considered $[7,8]$, and depends on the interplay of gravitational particle production of field quanta, and the redshifting of created quanta.

For this reason we are motivated to study quantum effects in power-law inflation $[9,10]$, in which $0 \leq \epsilon=$ const. $<1$, as well as in more general slow roll inflationary models and beyond. Power-law inflation is excluded as a model of primordial inflation by the cosmic microwave background observations [11], and it does not capture the effect of an evolving principal slow-roll parameter. Nevertheless, it should be a better background model than de Sitter for studying quantum loop effects in the primordial universe, as it does capture the effect of a non-vanishing principal slow-roll parameter, and notably, it is still tractable enough to allow for analytic computations. Thus far there have been several reports on loop computations in power-law inflation, and general slow-roll inflation, some utilizing perturbative methods [7, 12-15], and some employing resummation/non-perturbative techniques [16-18]. Noteworthy is also the recent construction [19] of the one-loop effective potential in general inflationary models in which the principal slow roll parameter $\epsilon(t)$ is a general function of time. 
Gauge fields couple conformally to gravity, and by themselves they are not sensitive to the expansion of the Universe. ${ }^{1}$ However, the effects of the expansion can be mediated to them by coupling to either gravitons or to light scalars. This happens already at tree level if the scalar develops a condensate which breaks confomal invariance of the gauge fields, or at the loop level where conformal symmetry is broken due to infrared (IR) fluctuations of the graviton or the light scalar. The former case corresponds to the mechanism of spontaneous symmetry breaking. In the latter case inflation generates a medium of long wavelength quantum fluctuations in which a quantum field can lose its classical conformal symmetry, which corresponds to the mechanism of dynamical symmetry breaking. One of the simplest, and arguably most interesting, examples of such a phenomenon is the photon dynamics in presence of a light, charged scalar in inflation. The simplest, and most striking, manifestation of the local conformal symmetry breaking is the photon mass generation in scalar electrodynamics in de Sitter space [24-26]. Even though it originates from quantum loops, this mass contributes to the effective action as a local, tree-level, mass term, akin to the mass in the Brout-Englert-Higgs (BEH) mechanism that operates in the standard model. However, contrary to the mass generation in the standard model, which is mediated by the classical Higgs condensate, the inflationary mass generation is predominantly due to the long wavelength (super-Hubble) fluctuations of a light (or massless) charged scalar. Nevertheless, at the level of the effective action the description of the two phenomena is identical, when treated in the adiabatic approximation.

Motivated by these insights, in this work we study the non-minimally coupled Abelian Higgs model in power-law inflation, a particular instance of scalar electrodynamics in which the mass term of the complex scalar $\Phi=\frac{1}{\sqrt{2}}(\phi+i \chi)$ is substituted by the non-minimal coupling $\xi$ to the Ricci curvature scalar $R$,

$$
\begin{aligned}
S\left[A_{\mu}, \Phi, \Phi^{*}\right]=\int d^{D} x \sqrt{-g}[ & -\frac{1}{4} g^{\mu \rho} g^{\nu \sigma} F_{\mu \nu} F_{\rho \sigma} \\
& \left.-g^{\mu \nu}\left(D_{\mu}^{*} \Phi^{*}\right)\left(D_{\nu} \Phi\right)-\lambda\left(\Phi^{*} \Phi\right)^{4}-\xi R \Phi^{*} \Phi\right] .
\end{aligned}
$$

Here $D_{\mu}=\partial_{\mu}+i q A_{\mu}$ is the $\mathrm{U}(1)$ covariant derivative, $q$ the $\mathrm{U}(1)$ charge, and $F_{\mu \nu}=$ $\partial_{\mu} A_{\nu}-\partial_{\nu} A_{\mu}$ is the field strength tensor associated with the vector potential $A_{\mu}$. The action is written in $D$ space-time dimensions to facilitate dimensionally regulated quantum loop computations. There are multiple reasons for studying this particular model. Firstly, it allows one to study the effects of the time-dependent scalar condensate in the attractor regime of power-law inflation, where both the expansion of the universe and the evolution of the condensate play a significant role. Secondly, the model in (1.2) can be used to describe the effects that the infrared quantum fluctuations of a light scalar impart on the vector field they couple to [27]. Thirdly, the action (1.2) can be used as a toy model for the

\footnotetext{
${ }^{1}$ The classical local conformal (Weyl) symmetry is broken by quantum loop effects via the conformal anomaly [20, 21], and thus the conformally coupled fields can feel the expansion of the Universe without coupling to the light scalar or the graviton. However, the effect of the anomaly on the vector field dynamics is typically quite small $[22,23]$.
} 
electroweak sector of the standard model both in inflationary as well as in post-inflationary epochs. In what follows we elaborate on the former two.

Complex scalar condensate. The evolution of spectator scalar fields ${ }^{2}$ in inflation often exhibits attractor regimes, where information about initial conditions is lost. In powerlaw inflation, apart from the symmetric solution with a vanishing condensate $\Phi=0$, the complex scalar field of the model in (1.2) can develop a symmetry-breaking condensate that traces the evolution of the Hubble rate $\Phi \propto H$, which in power-law inflation evolves according to $-\dot{H} / H^{2}=\epsilon=$ const. This attractor solution has been considered before in the literature $[8,13,28]$. The vector field, which is assumed not to have a condensate, is conformally coupled to gravity at tree-level in the absence of a scalar condensate. The non-vanishing scalar condensate is responsible for the spontaneous symmetry breaking mechanism, as it induces a mass-like term for the vector field, which is more properly seen as an effective non-minimal coupling of the vector field to the Ricci curvature scalar, on the account of its time-dependence tracing the evolution of the Hubble rate,

$$
\left(\Phi^{*} \Phi\right) g^{\mu \nu} A_{\mu} A_{\nu} \propto H^{2} g^{\mu \nu} A_{\mu} A_{\nu} \propto R g^{\mu \nu} A_{\mu} A_{\nu}
$$

This is how the conformal coupling of the vector to gravity is broken in the symmetrybreaking attractor solution in power-law inflation, and how the vector becomes sensitive to the expansion of space-time at tree-level.

The fluctuations of the complex scalar around the condensate already are nonminimally coupled to gravity in (1.2), but the presence of a condensate effectively renormalizes the coupling constant (see section 5 for more details). Since the non-minimal coupling can be seen as a time-dependent mass term, one expects similarities with the Abelian Higgs model in flat space that develops time-dependent masses. This work is mostly devoted to quantifying the effects that the time-dependent condensate (1.3) has on the linear quantum fluctuations of the Abelian Higgs model in (1.2). This is accomplished by working out the propagators of both scalar and vector linear fluctuations, thus setting up the stage for studying the loop corrections to the inflationary dynamics of the model.

Infrared scalar fluctuations. In scalar electrodynamics models where the complex scalar does not develop a condensate the vector field remains massless at tree-level in cosmological spaces, and is not sensitive to the expansion. However, a light complex scalar can induce a mass of the vector purely due to large infrared (super-Hubble) quantum fluctuations. In refs. [24, 25, 29] a perturbative one-loop analysis was carried out in de Sitter assuming a massless scalar. The one-loop vacuum polarization was computed in ref. [24], and it was found that the correction terms it provides to the effective equation of motion for the vector potential mode function at late times takes the form of a momentumdependent mass term,

$$
m_{A}^{2}=\frac{(q H)^{2}}{2 \pi^{2}} \times \ln \left(\frac{k}{H}\right)
$$

\footnotetext{
${ }^{2}$ Spectator fields in inflation are the ones whose backreaction on the expansion rate can be neglected.
} 
where $k$ is the modulus of the mode wave vector. The effective mode function equation was then solved in ref. [30], where it was found that the electric field gets secularly enhanced compared to the vacuum case, $E^{i} \sim a^{-3 / 2}$, while the magnetic field receives a correction to its amplitude, but the scaling remains that of the conformal vacuum, $B^{i} \sim a^{-2}$. The effect on the electric field can be effectively captured by a vector field with a secularly growing mass $m_{A} \sim \ln (a)$, but this local approximation to the vacuum polarization gives too little magnetic field. The subsequent work [26] adapted the analysis to the case of a light non-minimally coupled scalar, reporting an effective vector mass generation,

$$
m_{A}^{2}=\frac{(q H)^{2}}{2 \pi^{2}} \times \frac{3 H^{2}}{2\left(m_{\phi}^{2}+12 \xi H^{2}\right)},
$$

where $m_{\phi} \ll H$ is the scalar mass, and $\xi$ is its non-minimal coupling constant.

Perturbative studies [24-26] of vector mass generation by a massless/light complex scalar all pointed to the need for non-perturbative studies. By making a suitable generalization of Starobinsky's stochastic formalism [31], ref. [27] showed that, after a sufficiently long time, massless scalar quantum electrodynamics in de Sitter space settles to a nonperturbative, interacting vacuum state, in which the scalar remains perturbatively light, but the vector develops a non-perturbatively large masss, respectively,

$$
m_{\phi}^{2} \approx 0.45 \times \frac{(q H)^{2}}{4 \pi^{2}}, \quad m_{A}^{2} \approx 3.3 \times H^{2},
$$

where $m_{A}$ is the mass which characterizes the coincident vector propagator. This interacting vacuum state has lower energy than any free Gaussian state. Curiously, inserting the non-perturbative scalar mass (1.6) into (1.5) gives for the vector mass $m_{A}^{2} \simeq 2.2 \times H^{2}$, which is not far from its true value given in (1.6). This $\sim 30 \%$ difference can be attributed to the non-Gaussian nature of the interacting vacuum state. The masses in (1.6) are generated by the large infrared quantum fluctuations, i.e. by the composite operators developing a condensate, and thus are a result of a dynamical symmetry-breaking mechanism in de Sitter space. Vector field perturbations in de Sitter inflationary setting can affect inflationary observables [32, 33] and the dynamics of the standard model during inflation [34], which may have observable ramifications, thus warranting a closer investigation.

It would be very interesting to understand how the dynamical symmetry breaking picture from de Sitter space generalizes to more general cosmological spaces, such as powerlaw inflation considered here. To this end, we draw inspiration from the similarities between the spontaneous and dynamical symmetry-breaking mechanisms, in particular from the scaling of the scalar condensate $\langle\hat{\Phi}\rangle \propto H$ in the first case, and the presumed scaling of the scalar fluctuations $\left\langle\hat{\Phi}^{\dagger} \hat{\Phi}\right\rangle \propto H^{2}$ in the second case. The latter scaling is supported by the non-perturbative results pertaining to power-law inflationary dynamics of a real scalar field $\phi$ with a quartic self-interaction term, $\lambda \phi^{4} / 4$. In refs. [16, 17] the stochastic formalism [31] was considered in power-law inflation, where it was found that, at late times, the scalar variance indeed scales as $H^{2}$,

$$
\left\langle\hat{\phi}^{2}\right\rangle=H^{2} \int_{-\infty}^{\infty} d \sigma \sigma^{2} \rho(\sigma), \quad \rho(\sigma)=\rho_{0} \exp \left[\frac{-2}{(3-\epsilon) A}\left(\frac{\lambda}{4} \sigma^{4}-\frac{(3-\epsilon) \epsilon}{2} \sigma^{2}\right)\right]
$$


where $\sigma$ is a dimensionless integration variable, $\rho_{0}$ and $A$ are dimensionless constants that depend on $\epsilon$ and $\lambda$, where $\lambda$ is the quartic self-coupling constant, and $\sigma$ is a dimensionless integration variable. It is interesting that $\epsilon>0$ generates a negative correction to the effective mass term $\propto \epsilon$ appearing in the probability distribution function $\rho(\sigma)$. The scaling in (1.7) was further corroborated for the same model in ref. [18] by solving the gap equation resulting from the 2PI Dyson-Schwinger equation in power-law inflation.

The scaling of scalar fluctuations in the scalar electrodynamics model $\left\langle\hat{\Phi} \hat{\Phi}^{\dagger}\right\rangle \propto H^{2}$ is qualitatively different from the de Sitter case, where this quantity is just a constant, and if correct might account for interesting effects imparted on the vector. We cannot confirm that this is the case without a detailed computation, which indeed would be worthwhile pursuing. Furthermore, the vector propagators in cosmological spaces, both the massive one constructed here and the massless one left for future work, constitute fundamental building blocks for studying physical effects from the interactions of gauge fields with matter and gravity in inflation. Examples illustrating their importance are such phenomena as large scale cosmological magnetic and electric fields [7, 22, 30, 35-41], generation of gravitational waves [42-45], etc.

Perturbative studies may, however, not be enough to quantify interesting effects, and in order to fully capture the late time dynamics of interacting quantum fields in inflation, one may have to resort to non-perturbative methods. At present two such reliable methods are known, Starobinsky's stochastic inflation [31] and functional renormalization group methods [46]. It is known that the non-perturbative infrared effects are strong enough to restore symmetries during inflation. For example, in both the scalar field theory with a global $O(N)$ symmetry and in the real scalar self-interacting theory, an initially broken symmetry gets restored [47-49] and the scalars acquire a calculable mass (see ref. [13] for an earlier, perturbative analysis of the same problem). There are two works known to us in which symmetry restoration in theories involving gauge fields is investigated in de Sitter by means of non-perturbative methods: ref. $[27]^{3}$ uses stochastic methods and ref. [50] uses functional renormalization group methods to study the late time dynamics of the vector and scalar fields of scalar electrodynamics in de Sitter. These works found that, at late times, the symmetry gets restored and the vector and the scalar acquire a calculable mass (1.6). Both of the non-perturbative approaches in these studies utilized the massive vector field propagator in de Sitter space to capture the interactions between the two fields. For analogous studies in power-law inflation it is important to have the massive vector field propagator that we construct here.

Gauge choice. The Abelian Higgs model in (1.2) that we consider is a gauge theory, and its quantization necessarily requires fixing a gauge. In this work we impose the unitary gauge, which we implement using Dirac's formalism for systems with first-class constraints [51-53]. This amounts to enforcing the condition $\operatorname{Im}(\Phi)=0$ that fully fixes the gauge. Here we opt for the unitary gauge for two reasons: (i) it is physically transparent, as it allows for explicit reduction of phase space leaving two fields - a massive/non-minimally

\footnotetext{
${ }^{3}$ Even though ref. [27] never mentions that the results presented can be used in studies of symmetry restoration, the universality of the asymptotically late-time scalar and vector field masses implies that.
} 
coupled scalar and a massive/non-minimally coupled vector, and (ii) it leads to analytically tractable equations, and allows us to work out the two-point functions analytically. The form that the propagators take is simple enough to allow for explicit loop computations.

The propagators in the unitary gauge are constructed here with the intent of eventually employing them in loop computations. Having that in mind, we should point out that in flat space loop computations the unitary gauge is usually disfavoured compared to the U(1) variant of 't Hooft background field gauge, also known as the $R_{\xi}$ gauge $[54,55]$ which is an average gauge, determined at tree level by the gauge-fixing functional,

$$
S_{\mathrm{gf}}\left[A_{\mu}, \chi\right]=\int d^{D} x \sqrt{-g}\left[-\frac{1}{2 \alpha}\left(g^{\mu \nu} \nabla_{\mu} A_{\nu}-\alpha q \bar{\phi} \chi\right)^{2}\right],
$$

where $\alpha$ is an arbitrary gauge-fixing parameter, ${ }^{4}$ and where the complex scalar is split into its real and imaginary parts, $\Phi=[(\bar{\phi}+\varphi)+i \chi] / \sqrt{2}$, with $\bar{\phi}$ being the scalar condensate. The reasons why the $R_{\xi}$ gauge is favoured are: (i) The unitary gauge cannot be applied to the symmetric phase where the condensate vanishes, which can lead to some-non-perturbative issues [56]; (ii) The vector propagator in the unitary gauge is more singular than the propagator in the $R_{\xi}$ gauge, which makes the perturbation theory not power-counting renormalizable, and in fact requires additional counterterms be added to the effective action at each perturbative order to ensure renormalizability. The additional counterterms in flat space can be resummed into a single term, $\delta \mathscr{L}=i \delta^{4}(0) \ln (|\Phi| / \bar{\phi})$, which was first derived using canonical quantization methods [57-59], and subsequently by path-integral methods [60-64], thus establishing the connection with the BPHZ (Bogolyubov-ParasiukHepp-Zimmermann) renormalizability [65-67] which is known to hold in $R_{\xi}$ gauges [54]. Furthermore, it was shown in ref. [68] that the unitary gauge yields correct results for the on-shell ( $S$-matrix) calculations. Recently, a simple proof of renormalizability of the unitary gauge was provided in ref. [69], by making use of the background field method (in which the gauge fixing term is gauge invariant under the background field gauge transformations).

Of course, in flat space one has the luxury of choosing between different propagators at disposal, as is also the case in de Sitter space. However, in power-law inflation no massive vector propagators have been reported thus far, and one either needs to construct the vector propagator in the covariantized $R_{\xi}$ gauge, or deal with the three issues mentioned above when using the propagator reported here. We think that these issues will not represent an obstacle if the unitary gauge vector propagator is employed with due care. The issue in (i) is not worrying since non-perturbative issues do not arise in perturbative computations in the broken phase. The renormalization issue in (ii) does appear in perturbative computations, and needs to be addressed, even more so having in mind that the additional counterterms have been worked out only for flat space. The necessity of additional counterterms ultimately derives from the fact that the Feynman propagator in the unitary gauge is not the Green's function, but the two differ by a non-covariant local term. This difference between the two, which does not usually appear in quantum field theories, follows from the rules of canonical quantization in the unitary gauge [57, 70]. If one insists

\footnotetext{
${ }^{4}$ We denote the gauge-fixing parameter of the $R_{\xi}$ gauge by $\alpha$ instead of the more common $\xi$, in order not to confuse it with the non-minimal coupling we denote by $\xi$.
} 
on using just the covariant Green's function in the loop expansion as customary, this is the reason behind the necessity of additional counterterms [57, 58]. When the distinction between the two is maintained in the perturbative expansion, which can be inferred by using the Yang-Feldman equation [70, 71], the additional counterterms should not be required. This is particularly important for the applications we have in mind, as it is not clear how to generalize the extra counterterms to power-law inflation. That is why we pay great attention to the distinction between the Feynman propagator and the Green's function in section 7 , and we carefully work out both, in addition to the Wightman functions.

Even though we believe that the unitary gauge propagators can be used in perturbative computations in power-law inflation, it would be beneficial to have the propagators worked out in the $R_{\xi}$ gauge as well. Those could then be applied to the studies of massless vector fields as well, but even more importantly, we would have a free gauge-fixing parameter which would allow to explicitly test the computed observables for gauge-dependence. This is of a crucial importance for quantum field theory in cosmological spaces, where the $S$ matrix either does not exist, or is of limited utility for the initial-value problems, and one is forced to construct observables which are real-time expectation values, for which the Schwinger-Keldysh formalism [72, 73] is suitable. However, one big advantage of the $R_{\xi}$ gauge is lost when the scalar condensate acquires space-time dependence, $\partial_{\mu} \bar{\phi} \neq 0$. In that case the vector field no longer decouples from the Goldstone boson $\chi[74,75]$ at tree level, which complicates the analysis and requires the use of the mixed field (scalar-vector) propagators in loop computations. Therefore, one would have to either come up with a modification of the gauge-fixing functional (1.8) which preserves the decoupling property, or work out the mixed field propagators as well.

We should also mention that the unitary gauge is often referred to as the $\alpha \rightarrow \infty$ limit of the $R_{\xi}$ gauge (1.8), in which the Goldstone boson $\chi$ becomes infinitely massive and decouples. This indeed is true for the most part, except for a subtle, but important fact that taking this limit does not commute with loop integrations in the perturbative expansion [74]. The origin of this issue can be traced to the difference between the Feynman propagator and the Green's function. In fact, the limit $\alpha \rightarrow \infty$ of the $R_{\xi}$ gauge produces the Green's function of the unitary gauge, not the Feynman propagator.

Related work. Not much is known about gauge field propagators in general cosmological spaces, and in this regard the best understood is de Sitter space, which is the model space for cosmological inflation. There are several results in de Sitter space related to our work. The massive vector field propagator in the unitary gauge was constructed by Allen and Jacobson in ref. [76]. Tsamis and Woodard [78] constructed the massive vector propagator in de Sitter in the Landau gauge limit of the $R_{\xi}$ gauge, in which the propagator is exactly transverse. Fröb and Higuchi [79] constructed the massive vector propagator in de Sitter in the Stueckelberg model, in the appropriate adaptation of the $R_{\xi}$ gauge, which was rederived for a particular choice of the gauge-fixing parameter in [80]. The Stueckelberg model in de Sitter space can be seen to match the linearized Abelian Higgs model where the condensate takes a constant value, and thus reproduces the Allen-Jacobson propagator in the unitary gauge limit. The principal goal of this work is to generalize the above results 
and construct the massive vector propagator for power-law inflation. The Tsamis-Woodard vector propagator [78] admits a regular massless limit, and is the only one that has been successfully utilized in loop computations for scalar electrodynamics [27, 50, 81], and for electromagnetism interacting with dynamical gravity [83, 84].

Outline. The paper is organized as follows. In sections 2 and 3 we discuss the background fields in power-law inflation and introduce scalar electrodynamics, the model for which the massive photon propagator is constructed in this work. Since the cosmological literature is exiguous regarding the delicate steps involved in the quantization of systems with constraints such as gauge theories, in section 3 we present in some detail the classical analysis of the constraints and construct the (extended) Hamiltonian, the corresponding action, and calculate the Dirac brackets. Next, in section 4 we discuss the dynamics of the scalar condensate, and in section 5 the propagator for scalar perturbations is briefly discussed. In section 6 the Dirac-Bergmann quantization [51-53, 85-87] is applied to the model at hand, whereby a special attention is devoted to how to obtain the unitary gauge within that general procedure. The main result is obtained in section 7 , where we construct the covariant vector propagator, the Green function and the Wightman two-point function, which are the basic building blocks of perturbation theory in the Schwinger-Keldysh formalism [72, 73]. We check our vector propagator by considering various limits, which include Minkowski and de Sitter space, and compare with the available known results. The vector propagators are put to use in section 8 where we compute the field strength correlators and discuss the behavior of the equal-time electric and magnetic field correlators at super-Hubble separations. Finally, in section 9 we discuss our main results and outline future perspectives. Some important technical details are relegated to appendices.

\section{FLRW and power-law inflation}

The spatially flat ${ }^{5}$ Friedmann-Lemaître-Robertson-Walker (FLRW) space-time is a spatially homogeneous and isotropic expanding space, given by the invariant line element,

$$
d s^{2}=g_{\mu \nu}(x) d x^{\mu} d x^{\nu}=-d t^{2}+a^{2}(t) d \vec{x}^{2}=a^{2}(\eta)\left[-d t^{2}+d \vec{x}^{2}\right],
$$

where $t$ is the physical (comoving) time, $\eta$ is the conformal time, $\vec{x}=\left(x^{1}, x^{2}, \cdots, x^{D-1}\right)$ denote the comoving spatial coordinates, and $a$ is the scale factor. In this work we prefer to use conformal time in which FLRW background is given by a conformally flat metric,

$$
g_{\mu \nu}=a^{2}(\eta) \eta_{\mu \nu}
$$

where $\eta_{\mu \nu}=\operatorname{diag}(-1,1, \ldots, 1)$ is the $D$-dimensional Minkowski metric. The rate of the expansion is encoded by the conformal Hubble rate, or by the physical Hubble rate, respectively,

$$
\mathcal{H}=\frac{\partial_{0} a}{a}, \quad H=\frac{\mathcal{H}}{a},
$$

\footnotetext{
${ }^{5}$ The assumption of spatial flatness is consistent with all current observations $[11,88]$.
} 
where $\partial_{0}=\partial / \partial x^{0}=\partial / \partial \eta$ denotes a derivative with respect to conformal time. We make frequent use of both Hubble rates. The acceleration rate is conveniently parametrized by the principal slow-roll parameter,

$$
\epsilon=1-\frac{\partial_{0} \mathcal{H}}{\mathcal{H}^{2}}
$$

which is related to the deceleration parameter $q=\epsilon-1$. When $\epsilon<1$ the universe is accelerating, and when $\epsilon>1$ it is decelerating.

In this work we study the Abelian Higgs model in power-law inflation, which is characterized by a constant $\epsilon$ parameter in the accelerating range,

$$
\epsilon=\text { constant } \quad \& \quad 0 \leq \epsilon<1 \quad \text { (power law inflation), }
$$

where $\epsilon=0$ corresponds to de Sitter space-time (more precisely to the Poincaré patch of de Sitter). The scale factor and the conformal Hubble rate in power-law inflation are given by,

$$
a=\left[1-(1-\epsilon) H_{0}\left(\eta-\eta_{0}\right)\right]^{\frac{-1}{1-\epsilon}}, \quad \mathcal{H}=H_{0}\left[1-(1-\epsilon) H_{0}\left(\eta-\eta_{0}\right)\right]^{-1}, \quad \mathcal{H} a^{\epsilon-1}=H_{0},
$$

where $\eta_{0}$ is some initial time, $H_{0}=\mathcal{H}\left(\eta_{0}\right)$, and $a\left(\eta_{0}\right)=1$. The ranges of coordinates in power-law inflation are

$$
-\infty<\eta<\eta_{0}+\frac{1}{(1-\epsilon) H_{0}} \equiv \bar{\eta}, \quad-\infty<x^{i}<\infty,
$$

and the conformal diagram of its causal structure is given in figure 1 . The curvature tensors ${ }^{6}$ in power-law inflation are given by,

$$
\begin{aligned}
R_{\mu \nu \rho \sigma} & =2 H^{2} g_{\mu[\rho} g_{\sigma] \nu}+4 \epsilon H^{2}\left(a^{2} \delta_{[\mu}^{0} g_{\nu][\sigma} \delta_{\rho]}^{0}\right), \\
R_{\mu \nu} & =(D-1-\epsilon) H^{2} g_{\mu \nu}+(D-2) \epsilon H^{2}\left(a^{2} \delta_{\mu}^{0} \delta_{\nu}^{0}\right), \\
R & =(D-1)(D-2 \epsilon) H^{2},
\end{aligned}
$$

We make a frequent use of the classical distance function between two points $x$ and $x^{\prime}$,

$$
y\left(x ; x^{\prime}\right)=(1-\epsilon)^{2} \mathcal{H} \mathcal{H}^{\prime} \Delta x^{2},
$$

where $\Delta x^{2}=\Delta x^{2}\left(x ; x^{\prime}\right)$ is the geodesic distance in the conformal Minkowski space-time,

$$
\Delta x^{2}=\eta_{\mu \nu}\left(x-x^{\prime}\right)^{\mu}\left(x-x^{\prime}\right)^{\nu}=\left\|\vec{x}-\vec{x}^{\prime}\right\|^{2}-\left(\eta-\eta^{\prime}\right)^{2} \equiv\|\Delta \vec{x}\|^{2}-(\Delta \eta)^{2} .
$$

In the de Sitter limit, where $\epsilon=0$, this function is related to the geodesic distance $\ell\left(x ; x^{\prime}\right)$ between two points $x$ and $x^{\prime}$ as $\left.y\left(x ; x^{\prime}\right)\right|_{\epsilon=0}=4 \sin ^{2}\left[H_{0} \ell\left(x ; x^{\prime}\right) / 2\right]$. In power-law inflation this relation no longer holds, but it is still very convenient to express our results in terms of $y\left(x ; x^{\prime}\right)$.

In the quantum theory, the two-point functions will depend on the distance function (2.11), and their distributional character will be conveniently encoded by suitable

\footnotetext{
${ }^{6}$ The conventions that we use are $R_{\mu \beta \nu}^{\alpha}=\partial_{\beta} \Gamma_{\mu \nu}^{\alpha}-\partial_{\nu} \Gamma_{\mu \beta}^{\alpha}+\Gamma_{\mu \nu}^{\rho} \Gamma_{\beta \rho}^{\alpha}-\Gamma_{\mu \beta}^{\rho} \Gamma_{\nu \rho}^{\alpha}$ for the Riemann tensor, and $\Gamma_{\mu \nu}^{\alpha}=\frac{1}{2} g^{\alpha \beta}\left(\partial_{\mu} g_{\nu \beta}+\partial_{\nu} g_{\mu \beta}-\partial_{\beta} g_{\mu \nu}\right)$ for the Christoffel symbol.
} 


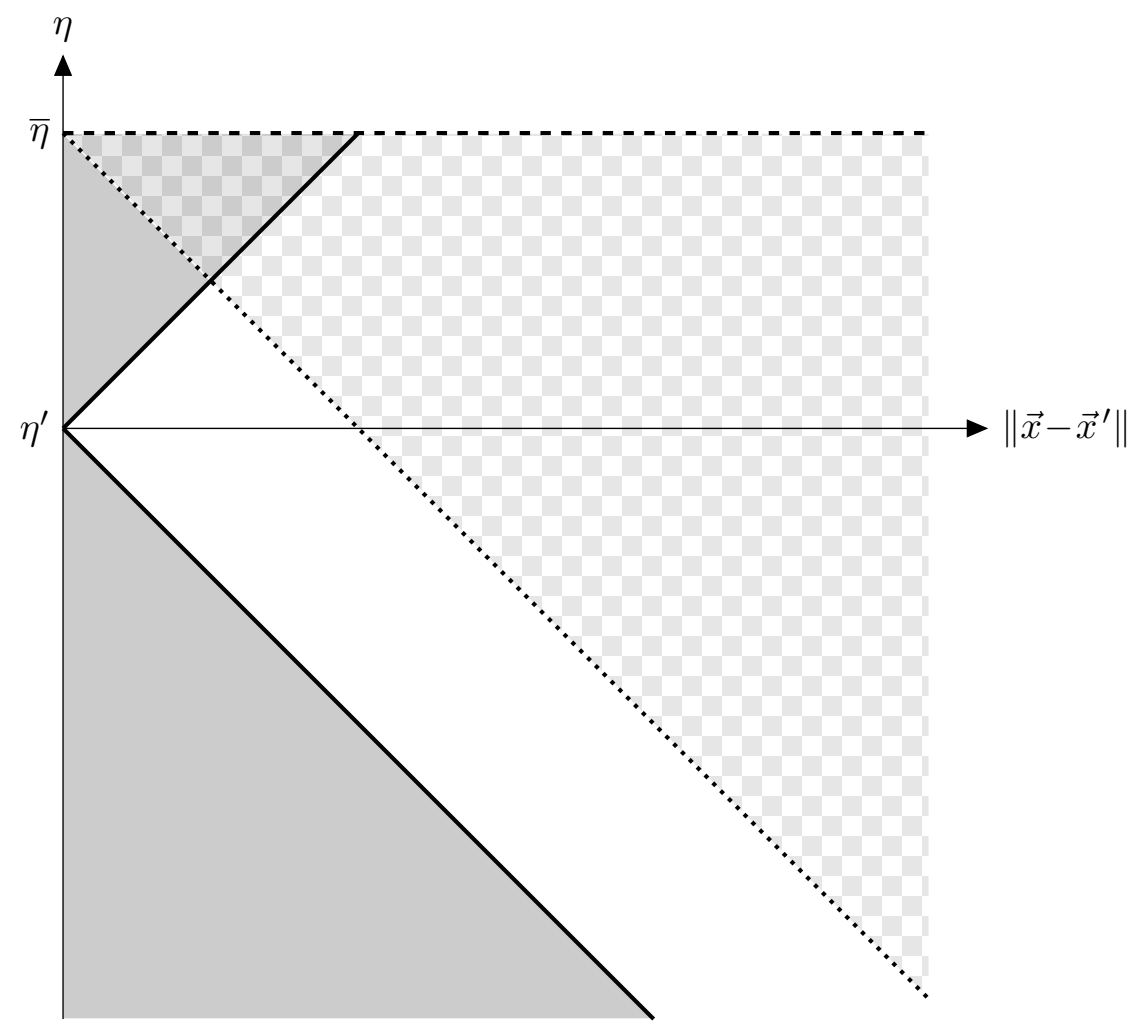

Figure 1. The conformal diagram of power-law inflation. Thick solid lines represent the past and future null rays of some point $\left(\eta^{\prime}, \vec{x}^{\prime}\right)$, while the gray shaded regions represent the past and future light-cones of that point. In the conformal coordinates the past light-cone grows without limits, while the future light-cone is limited by $\bar{\eta}$ from (2.7), that corresponds to the asymptotic future. Dotted line denotes the Hubble horizon, and the checkered region the points that are causally disconnected from the observer at $\vec{x}^{\prime}$.

imaginary shifts of time in the distance function. All relevant two-point functions can be obtained from the following $i \varepsilon$-prescriptions [89],

$$
y_{A B}\left(x ; x^{\prime}\right)=(1-\epsilon)^{2} \mathcal{H H}^{\prime} \Delta x_{A B}^{2}, \quad A, B= \pm,
$$

where the prescription lies entirely within the conformal Minkowski part, ${ }^{7}$

$$
\begin{array}{ll}
\Delta x_{++}^{2}=\left\|\vec{x}-\vec{x}^{\prime}\right\|^{2}-\left(\left|\eta-\eta^{\prime}\right|-i \varepsilon\right)^{2}, & \Delta x_{--}^{2}=\left(\Delta x_{++}^{2}\right)^{*}, \\
\Delta x_{-+}^{2}=\left\|\vec{x}-\vec{x}^{\prime}\right\|^{2}-\left(\eta-\eta^{\prime}-i \varepsilon\right)^{2}, & \Delta x_{+-}^{2}=\left(\Delta x_{-+}^{2}\right)^{*},
\end{array}
$$

\footnotetext{
${ }^{7}$ Note there are different ways of defining $i \varepsilon$-prescriptions corresponding to given two-point functions, e.g.

$$
\Delta x_{++}^{2}=\left\|\vec{x}-\vec{x}^{\prime}\right\|^{2}-\left(\eta-\eta^{\prime}\right)^{2}-i \varepsilon, \quad \Delta x_{-+}^{2}=\left\|\vec{x}-\vec{x}^{\prime}\right\|^{2}-\left(\eta-\eta^{\prime}\right)^{2}-i \varepsilon \operatorname{sgn}\left(\eta-\eta^{\prime}\right),
$$

as employed in e.g. [90]. This reflects the fact that defining a distribution in terms of a limit of an analytic function is not unique. One needs to be careful to use prescriptions consistently, as some expressions involving derivatives may seem rather different in different prescriptions. The prescription (2.14)-(2.15) we use here naturally arises when the scalar propagator is constructed as a sum over modes [8].
} 
The $( \pm)$ nomenclature is adapted to the Schwinger-Keldysh formalism [72, 73, 91, 92], and refers to different types of two-point functions. Namely, $(++)$ refers to the Feynman, and $(--)$ to the Dyson propagator, while $(-+)$ and $(+-)$ refer to the positive- and negativefrequency Wightman functions, respectively. Note that distance functions with different $i \varepsilon-$ prescriptions are not all independent, in particular,

$$
y_{++}=\theta\left(\eta-\eta^{\prime}\right) y_{-+}+\theta\left(\eta^{\prime}-\eta\right) y_{+-}
$$

\section{Abelian Higgs model in cosmological spaces}

In this section we consider non-minimally coupled Abelian Higgs model on an expanding FLRW background, and derive its canonical formulation. We discuss the gauge-fixing procedure according to the Dirac-Bergmann algorithm [51-53, 85-87], and fix the unitary gauge. We then consider the perturbations in this model around a background of expanding space-time with an evolving homogeneous and isotropic scalar condensate. The Hamiltonian operator for the linearized perturbations is identified, as well as successively higher order interaction Hamiltonians.

The action for the non-minimally coupled Abelian Higgs model we consider, involving a vector field $A_{\mu}$ and a complex scalar field $\Phi$, on a general curved background is given by

$$
S\left[A_{\mu}, \Phi, \Phi^{*}\right]=\int d^{D} x \sqrt{-g}\left[-\frac{1}{4} g^{\mu \rho} g^{\nu \sigma} F_{\mu \nu} F_{\rho \sigma}-g^{\mu \nu}\left(D_{\mu}^{*} \Phi^{*}\right)\left(D_{\nu} \Phi\right)-\lambda\left(\Phi^{*} \Phi\right)^{2}-\xi R \Phi^{*} \Phi\right],
$$

where $g_{\mu \nu}$ is the metric tensor of the background space-time, and $g=\operatorname{det}\left(g_{\mu \nu}\right)$ is the metric determinant. Furthermore, $F_{\mu \nu}=\partial_{\mu} A_{\nu}-\partial_{\nu} A_{\mu}$ is the vector field strength tensor, $D_{\mu}=\partial_{\mu}+i q A_{\mu}$ is the $\mathrm{U}(1)$ covariant derivative, $q$ is the $\mathrm{U}(1)$ charge, $\lambda$ is the scalar quartic self-coupling constant, and $\xi$ is the dimensionless non-minimal coupling constant between the scalar and the Ricci scalar $R$. This action is invariant under the $\mathrm{U}(1)$ gauge transformations,

$$
A_{\mu} \rightarrow A_{\mu}+\partial_{\mu} \Lambda, \quad \Phi \rightarrow e^{-i q \Lambda} \Phi, \quad \Phi^{*} \rightarrow e^{i q \Lambda} \Phi^{*},
$$

where $\Lambda$ is an arbitrary space-time dependent function. The action (3.1) specialized to the FLRW background reads,

$$
\begin{aligned}
S\left[A_{\mu}, \Phi, \Phi^{*}\right]=\int d^{D} x a^{D-4}[ & -\frac{1}{4} \eta^{\mu \rho} \eta^{\nu \sigma} F_{\mu \nu} F_{\rho \sigma}-a^{2} \eta^{\mu \nu}\left(D_{\mu}^{*} \Phi^{*}\right)\left(D_{\nu} \Phi\right) \\
& \left.-\lambda a^{4}\left(\Phi^{*} \Phi\right)^{2}-\xi(D-1)(D-2 \epsilon) a^{2} \mathcal{H}^{2} \Phi^{*} \Phi\right] .
\end{aligned}
$$

This model is expected to share some similarities with the Abelian Higgs model in flat space despite not containing the mass term, since in $D=4$ the rescaled field $\Phi / a$ is described by precisely the flat space action for the Abelian Higgs model with a time-dependent mass $\mathcal{M}^{2}=(6 \xi-1)(2-\epsilon) \mathcal{H}^{2}$, which encodes the cosmological expansion. 


\subsection{Hamiltonian formulation and gauge-fixing}

The Hamiltonian formulation of the Abelian Higgs model in (3.3), and the subsequent imposition of the unitary gauge, is more transparent if we split the complex scalar into its real and imaginary components, ${ }^{8}$

$$
\Phi=\frac{1}{\sqrt{2}}(\phi+i \chi), \quad \Phi^{*}=\frac{1}{\sqrt{2}}(\phi-i \chi),
$$

in terms of which the FLRW action (3.3) reads,

$$
\begin{aligned}
S\left[A_{\mu}, \phi, \chi\right]=\int d^{D} x a^{D-4}[ & -\frac{1}{4} \eta^{\mu \rho} \eta^{\nu \sigma} F_{\mu \nu} F_{\rho \sigma}-\frac{a^{2}}{2} \eta^{\mu \nu}\left(\partial_{\mu} \phi-q A_{\mu} \chi\right)\left(\partial_{\nu} \phi-q A_{\nu} \chi\right) \\
& -\frac{a^{2}}{2} \eta^{\mu \nu}\left(\partial_{\mu} \chi+q A_{\mu} \phi\right)\left(\partial_{\nu} \chi+q A_{\nu} \phi\right)-\frac{\lambda a^{4}}{4}\left(\phi^{2}+\chi^{2}\right)^{2} \\
& \left.-\frac{\xi}{2}(D-1)(D-2 \epsilon) a^{2} \mathcal{H}^{2}\left(\phi^{2}+\chi^{2}\right)\right] .
\end{aligned}
$$

When deriving the Hamiltonian formulation, it is also advantageous to decompose the indices into temporal and spatial ones, $A_{\mu}=\left(A_{0}, A_{i}\right)$ and $\partial_{\mu}=\left(\partial_{0}, \partial_{i}\right)$,

$$
\begin{array}{rl}
S\left[A_{0}, A_{i}, \phi, \chi\right]=\int d^{D} & x a^{D-4}\left[\frac{1}{2} F_{0 i} F_{0 i}-\frac{1}{4} F_{i j} F_{i j}+\frac{a^{2}}{2}\left(\partial_{0} \phi-q A_{0} \chi\right)^{2}+\frac{a^{2}}{2}\left(\partial_{0} \chi+q A_{0} \phi\right)^{2}\right. \\
& -\frac{a^{2}}{2}\left(\partial_{i} \phi-q A_{i} \chi\right)\left(\partial_{i} \phi-q A_{i} \chi\right)-\frac{a^{2}}{2}\left(\partial_{i} \chi+q A_{i} \phi\right)\left(\partial_{i} \chi+q A_{i} \phi\right) \\
& \left.-\frac{\lambda a^{4}}{4}\left(\phi^{2}+\chi^{2}\right)^{2}-\frac{\xi}{2}(D-1)(D-2 \epsilon) a^{2} \mathcal{H}^{2}\left(\phi^{2}+\chi^{2}\right)\right]
\end{array}
$$

and since $\eta_{i j}=\delta_{i j}=\eta^{i j}$, there is no need to distinguish between raised and lowered indices. Henceforth we write all the quantities with lower indices, where the summation over repeated indices is implied.

Next we introduce the extended action $\mathcal{S}$, which is only linear in first time derivatives. This is accomplished by promoting time derivatives to independent velocity fields,

$$
\partial_{0} A_{0} \rightarrow V_{0}, \quad F_{0 i} \rightarrow V_{i}, \quad\left(\partial_{0} \phi-q A_{0} \chi\right) \rightarrow v, \quad\left(\partial_{0} \chi+q A_{0} \phi\right) \rightarrow w,
$$

and introducing the accompanying Lagrange multipliers, $\Pi_{0}, \Pi_{i}, \pi$, and $\rho$, which ensure on-shell equivalence to the action $S$ we started with,

$$
\begin{aligned}
\mathcal{S}\left[A_{0}, V_{0},\right. & \left.\Pi_{0}, A_{i}, V_{i}, \Pi_{i}, \phi, v, \pi, \chi, w, \rho\right]=\int d^{D} x\left\{a ^ { D - 4 } \left[\frac{1}{2} V_{i} V_{i}-\frac{1}{4} F_{i j} F_{i j}\right.\right. \\
& +\frac{a^{2}}{2}\left(v^{2}+w^{2}\right)-\frac{a^{2}}{2}\left(\partial_{i} \phi-q A_{i} \chi\right)\left(\partial_{i} \phi-q A_{i} \chi\right)-\frac{a^{2}}{2}\left(\partial_{i} \chi+q A_{i} \phi\right)\left(\partial_{i} \chi+q A_{i} \phi\right) \\
& \left.-\frac{\lambda a^{4}}{4}\left(\phi^{2}+\chi^{2}\right)^{2}-\frac{\xi}{2}(D-1)(D-2 \epsilon) a^{2} \mathcal{H}^{2}\left(\phi^{2}+\chi^{2}\right)\right]+\Pi_{0}\left(\partial_{0} A_{0}-V_{0}\right) \\
& \left.+\Pi_{i}\left(F_{0 i}-V_{i}\right)+\pi\left(\partial_{0} \phi-q A_{0} \chi-v\right)+\rho\left(\partial_{0} \chi+q A_{0} \phi-w\right)\right\}
\end{aligned}
$$

\footnotetext{
${ }^{8}$ We employ the Cartesian coordinates for the complex scalar when discussing the unitary gauge, rather than the more customary polar coordinates. This is to emphasize that the singularity at the origin of the field space is not an artifact of the coordinate system used, but rather is a feature of the gauge condition itself.
} 
A variation of the extended action with respect to the velocity fields results in algebraic on-shell relations. ${ }^{9}$ We solve these for as many velocities as possible, which in this case means all except $V_{0}$,

$$
\begin{array}{llrl}
\frac{\delta \mathcal{S}}{\delta V_{i}}=a^{D-4} V_{i}-\Pi_{i} \approx 0 & \Rightarrow & V_{i} \approx \bar{V}_{i}=a^{4-D} \Pi_{i}, \\
\frac{\delta \mathcal{S}}{\delta v}=a^{D-2} v-\pi \approx 0 & \Rightarrow & v \approx \bar{v}=a^{2-D} \pi, \\
\frac{\delta \mathcal{S}}{\delta w}=a^{D-2} w-\rho \approx 0 & \Rightarrow & w \approx \bar{w}=a^{2-D} \rho .
\end{array}
$$

These on-shell relations are then plugged into the extended action (3.8) as off-shell equalities, which results in the canonical action $\mathscr{S}$,

$$
\begin{gathered}
\mathscr{S}\left[A_{0}, \Pi_{0}, A_{i}, \Pi_{i}, \phi, \pi, \chi, \rho, V_{0}\right] \equiv \mathcal{S}\left[A_{0}, V_{0}, \Pi_{0}, A_{i}, \bar{V}_{i}, \Pi_{i}, \phi, \bar{v}, \pi, \chi, \bar{w}, \rho\right] \\
=\int d^{D} x\left[\Pi_{0} \partial_{0} A_{0}+\Pi_{i} \partial_{0} A_{i}+\pi \partial_{0} \phi+\rho \partial_{0} \chi-\mathscr{H}-V_{0} \Psi_{1}\right]
\end{gathered}
$$

where the Hamiltonian density is

$$
\begin{aligned}
\mathscr{H}= & \frac{a^{4-D}}{2} \Pi_{i} \Pi_{i}+\Pi_{i} \partial_{i} A_{0}+\frac{a^{D-4}}{4} F_{i j} F_{i j}+\frac{a^{2-D}}{2}\left(\pi^{2}+\rho^{2}\right)+q A_{0}(\pi \chi-\rho \phi) \\
& +\frac{a^{D-2}}{2}\left(\partial_{i} \phi-q A_{i} \chi\right)\left(\partial_{i} \phi-q A_{i} \chi\right)+\frac{a^{D-2}}{2}\left(\partial_{i} \chi+q A_{i} \phi\right)\left(\partial_{i} \chi+q A_{i} \phi\right) \\
& +\frac{\lambda a^{D}}{4}\left(\phi^{2}+\chi^{2}\right)^{2}+\frac{\xi}{2}(D-1)(D-2 \epsilon) a^{D-2} \mathcal{H}^{2}\left(\phi^{2}+\chi^{2}\right),
\end{aligned}
$$

and $\Psi_{1}=\Pi_{0}$ is the primary constraint. Note that in the canonical action $V_{0}$ has an interpretation of a Lagrange multiplier, as the variation with respect to it generates the primary constraint. The Poisson brackets follow from the symplectic part of the canonical action (3.12), the non-vanishing ones being,

$$
\begin{aligned}
\left\{A_{0}(\eta, \vec{x}), \Pi_{0}\left(\eta, \vec{x}^{\prime}\right)\right\} & =\delta^{D-1}\left(\vec{x}-\vec{x}^{\prime}\right), \quad\left\{A_{i}(\eta, \vec{x}), \Pi_{j}\left(\eta, \vec{x}^{\prime}\right)\right\}=\delta_{i j} \delta^{D-1}\left(\vec{x}-\vec{x}^{\prime}\right), \\
\left\{\phi(\eta, \vec{x}), \pi\left(\eta, \vec{x}^{\prime}\right)\right\} & =\delta^{D-1}\left(\vec{x}-\vec{x}^{\prime}\right), \quad\left\{\chi(\eta, \vec{x}), \rho\left(\eta, \vec{x}^{\prime}\right)\right\}=\delta^{D-1}\left(\vec{x}-\vec{x}^{\prime}\right) .
\end{aligned}
$$

Note that the Lagrange multiplier $V_{0}$ is defined to have a vanishing Poisson bracket with all the canonical fields. The dynamics is generated by the total Hamiltonian,

$$
\mathrm{H}_{\text {tot }}=\int d^{D-1} x \mathscr{H}_{\text {tot }}, \quad \quad \mathscr{H}_{\text {tot }}=\mathscr{H}+V_{0} \Psi_{1}
$$

\footnotetext{
${ }^{9}$ Note that we are employing Dirac's notation, which distinguishes off-shell (strong) equalities denoted by the "=" symbol, and on-shell (weak) equalities denoted by the " $\approx "$ symbol.
} 
producing the Hamilton's equations,

$$
\begin{aligned}
\partial_{0} A_{0} \approx & V_{0} \\
\partial_{0} \Pi_{0} \approx & \partial_{i} \Pi_{i}-q(\pi \chi-\rho \phi) \\
\partial_{0} A_{i} \approx & a^{4-D} \Pi_{i}+\partial_{i} A_{0} \\
\partial_{0} \Pi_{i} \approx & a^{D-4} \partial_{j} F_{j i}+a^{D-2} q\left(\chi \partial_{i} \phi-\phi \partial_{i} \chi\right)-a^{D-2} q^{2}\left(\phi^{2}+\chi^{2}\right) A_{i} \\
\partial_{0} \phi \approx & a^{2-D} \pi+q A_{0} \chi \\
\partial_{0} \pi \approx & q A_{0} \rho+a^{D-2}\left(\nabla^{2} \phi-2 q A_{i} \partial_{i} \chi-q \chi \partial_{i} A_{i}-q^{2} \phi A_{i} A_{i}\right) \\
& -\lambda a^{D}\left(\phi^{2}+\chi^{2}\right) \phi-\xi(D-1)(D-2 \epsilon) a^{D-2} \mathcal{H}^{2} \phi \\
\partial_{0} \chi \approx & a^{2-D} \rho-q A_{0} \phi \\
\partial_{0} \rho \approx & -q A_{0} \pi+a^{D-2}\left(\nabla^{2} \chi+2 q A_{i} \partial_{i} \phi+q \phi \partial_{i} A_{i}-q^{2} \chi A_{i} A_{i}\right) \\
& -\lambda a^{D}\left(\phi^{2}+\chi^{2}\right) \chi-\xi(D-1)(D-2 \epsilon) a^{D-2} \mathcal{H}^{2} \chi
\end{aligned}
$$

which are to be supplemented with the primary constraint,

$$
\Psi_{1}=\Pi_{0} \approx 0 .
$$

Note that these are just the equations following from the canonical action (3.12), and that the Poisson brackets are defined in a way that reproduces them. The consistency of this primary constraint, i.e. its conservation in time, generates a secondary constraint,

$$
\partial_{0} \Psi_{1} \approx \partial_{i} \Pi_{i}-q(\pi \chi-\rho \phi) \quad \Rightarrow \quad \Psi_{2}=\partial_{i} \Pi_{i}-q(\pi \chi-\rho \phi) \approx 0 .
$$

The conservation of the secondary constraint generates no further constraints, rather it is conserved identically on-shell. All the Poisson brackets between the primary and the secondary constraints vanish,

$$
\left\{\Psi_{I}(\eta, \vec{x}), \Psi_{J}\left(\eta, \vec{x}^{\prime}\right)\right\}=0, \quad I, J=1,2,
$$

and thus they form a complete set of first-class constraints.

In order to fully determine the dynamics of the system additional conditions have to be supplied by hand. Namely, we need two additional constraints, $\Psi_{3} \approx 0$ and $\Psi_{4} \approx 0$, such that (i) they form a complete set of second-class constraints with the two first-class constraints (3.24) and (3.25),

$$
\operatorname{det}\left\{\Psi_{I}(\eta, \vec{x}), \Psi_{J}\left(\eta, \vec{x}^{\prime}\right)\right\} \not \approx 0, \quad I, J=1, \ldots, 4,
$$

and that (ii) they are consistent with the equations of motion (3.16)-(3.23). Satisfying the first condition is straightforward, but the second condition is more subtle, and in general does not allow us to chose the two gauge-fixing conditions independently. In this work we chose the unitary gauge, defined by requiring the scalar to be real,

$$
\Psi_{3}=\frac{1}{\sqrt{2}} \operatorname{Im}(\Phi)=\chi \approx 0 .
$$


It is sufficient to specify just this condition in order to fully fix the gauge. This is true because the second gauge condition is supplied by the conservation of the first one, required for consistency with the equations of motion,

$$
\partial_{0} \Psi_{3} \approx a^{2-D} \rho-q A_{0} \phi \quad \Rightarrow \quad \Psi_{4}=a^{2-D} \rho-q A_{0} \phi \approx 0 .
$$

The conservation of $\Psi_{4}$ generates no further constraints. The Poisson brackets between all four constraints $(I, J=1,2,3,4)$ form a matrix,

$$
\left\{\Psi_{I}(\eta, \vec{x}), \Psi_{J}\left(\eta, \vec{x}^{\prime}\right)\right\} \approx\left(\begin{array}{cccc}
0 & 0 & 0 & q \phi \\
0 & 0 & -q \phi & -a^{2-D} q \pi \\
0 & q \phi & 0 & a^{2-D} \\
-q \phi & a^{2-D} q \pi & -a^{2-D} & 0
\end{array}\right) \delta^{D-1}\left(\vec{x}-\vec{x}^{\prime}\right),
$$

with a non-vanishing determinant,

$$
\operatorname{det}\left\{\Psi_{I}(\eta, \vec{x}), \Psi_{J}\left(\eta, \vec{x}^{\prime}\right)\right\} \approx(q \phi)^{4} \times \delta^{D-1}\left(\vec{x}-\vec{x}^{\prime}\right) .
$$

This matrix is therefore invertible,

$$
\left\{\Psi_{I}(\eta, \vec{x}), \Psi_{J}\left(\eta, \vec{x}^{\prime}\right)\right\}^{-1} \approx\left(\begin{array}{cccc}
0 & a^{2-D} & a^{2-D} q \pi-q \phi \\
-a^{2-D} & 0 & q \phi & 0 \\
-a^{2-D} q \pi & -q \phi & 0 & 0 \\
q \phi & 0 & 0 & 0
\end{array}\right) \frac{1}{(q \phi)^{2}} \delta^{D-1}\left(\vec{x}-\vec{x}^{\prime}\right),
$$

and thus the four constraints - two first-class ones and two gauge conditions - form a complete set of second-class constraints. It is important to note that the unitary gauge is singular for $\phi=0$. In a quantized theory this is known to lead to some non-perturbative illnesses of the gauge [56]. However, for situations where $\phi$ develops a non-vanishing classical condensate, as is the case considered in this work, the unitary gauge suffices.

Having specified the gauge conditions, we may proceed, according to [51, 52], to determine the Dirac brackets, ${ }^{10}$ which are defined for two functions of canonical variables as,

$$
\begin{aligned}
& \left(\mid \mathrm{A}(\eta, \vec{x}), \mathrm{B}\left(\eta, \vec{x}^{\prime}\right)\right)=\left\{\mathrm{A}(\eta, \vec{x}), \mathrm{B}\left(\eta, \vec{x}^{\prime}\right)\right\} \\
& \quad-\int d^{D-1} z d^{D-1} z^{\prime}\left\{\mathrm{A}(\eta, \vec{x}), \Psi_{I}(\eta, \vec{z})\right\}\left\{\Psi_{I}(\eta, \vec{z}), \Psi_{J}\left(\eta, \vec{z}^{\prime}\right)\right\}^{-1}\left\{\Psi_{J}\left(\eta, \vec{z}^{\prime}\right), \mathrm{B}\left(\eta, \vec{x}^{\prime}\right)\right\},
\end{aligned}
$$

where the summation over the repeated constraint indices $I, J$ is implied. The Dirac brackets between the canonical variables for the case at hand are given in table 1. They serve the same role as Poisson brackets, with an important difference that the constraints may be imposed as off-shell equalities before computing any of the brackets, which corresponds to solving for the constraints. We use the four conditions (3.24), (3.25), (3.28), and (3.29) to solve for four canonical fields,

$$
\Pi_{0}=0, \quad \rho=-\frac{\left(\partial_{i} \Pi_{i}\right)}{q \phi}, \quad \chi=0, \quad A_{0}=-\frac{a^{2-D}\left(\partial_{i} \Pi_{i}\right)}{(q \phi)^{2}},
$$

\footnotetext{
${ }^{10}$ We denote the Dirac brackets by $(\cdot, \cdot)$ instead of the common symbol $\{\cdot, \cdot\}_{\mathrm{D}}$, which we find less distinguished and aesthetically less pleasing.
} 


\begin{tabular}{|c|cccccccc|}
\hline & $A_{0}\left(x^{\prime}\right)$ & $\Pi_{0}\left(x^{\prime}\right)$ & $A_{j}\left(x^{\prime}\right)$ & $\Pi_{j}\left(x^{\prime}\right)$ & $\phi\left(x^{\prime}\right)$ & $\pi\left(x^{\prime}\right)$ & $\chi\left(x^{\prime}\right)$ & $\rho\left(x^{\prime}\right)$ \\
\hline$A_{0}(x)$ & 0 & 0 & $\frac{a^{2-D}}{(q \phi)^{2}} \partial_{j}$ & 0 & 0 & $\frac{2 a^{2-D} \partial_{i} \Pi_{i}}{q^{2} \phi^{3}}$ & 0 & 0 \\
$\Pi_{0}(x)$ & 0 & 0 & 0 & 0 & 0 & 0 & 0 & 0 \\
$A_{i}(x)$ & $\partial_{i} \frac{a^{2-D}}{(q \phi)^{2}}$ & 0 & 0 & $\delta_{i j}$ & 0 & 0 & 0 & $\partial_{i} \frac{1}{q \phi}$ \\
$\Pi_{i}(x)$ & 0 & 0 & $-\delta_{i j}$ & 0 & 0 & 0 & 0 & 0 \\
$\phi(x)$ & 0 & 0 & 0 & 0 & 0 & 1 & 0 & 0 \\
$\pi(x)$ & $-\frac{2 a^{2-D} \partial_{i} \Pi_{i}}{q^{2} \phi^{3}}$ & 0 & 0 & 0 & -1 & 0 & 0 & $-\frac{\partial_{i} \Pi_{i}}{q \phi^{2}}$ \\
$\chi(x)$ & 0 & 0 & 0 & 0 & 0 & 0 & 0 & 0 \\
$\rho(x)$ & 0 & 0 & $\frac{1}{q \phi} \partial_{i}$ & 0 & 0 & $\frac{\partial_{i} \Pi_{i}}{q \phi^{2}}$ & 0 & 0 \\
\hline
\end{tabular}

Table 1. Coefficient operators $\mathrm{C}_{m, n}$ from Dirac brackets between canonical fields, $\left(\mid \mathrm{A}_{m}(\eta, \vec{x}), \mathrm{B}_{n}\left(\eta, \vec{x}^{\prime}\right)\right)=\mathrm{C}_{m, n} \times i \delta^{D-1}\left(\vec{x}-\vec{x}^{\prime}\right)$, where $\mathrm{A}_{m}$ is the entry from the leftmost column, $\mathrm{B}_{n}$ the entry from the topmost row, and $\mathrm{C}_{m, n}$ are the entries from the table. Unprimed derivatives are with respect to unprimed coordinates, and act on everything to the right of them.

so that we can define the reduced phase space Hamiltonian,

$$
\begin{aligned}
\mathrm{H}_{\mathrm{red}}=\int d^{D-1} x\left[\frac{a^{4-D}}{2} \Pi_{i} \Pi_{i}+\frac{a^{2-D}}{2} \frac{\left(\partial_{i} \Pi_{i}\right)\left(\partial_{j} \Pi_{j}\right)}{(q \phi)^{2}}+\frac{a^{D-4}}{4} F_{i j} F_{i j}+\frac{a^{D-2}}{2}(q \phi)^{2} A_{i} A_{i}\right. \\
\left.+\frac{a^{2-D}}{2} \pi^{2}+\frac{a^{D-2}}{2}\left(\partial_{i} \phi\right)\left(\partial_{i} \phi\right)+\frac{\lambda a^{D}}{4} \phi^{4}+\frac{\xi}{2}(D-1)(D-2 \epsilon) a^{D-2} \mathcal{H}^{2} \phi^{2}\right]
\end{aligned}
$$

When relations (3.34) are inserted into the Dirac brackets from table 1, one obtains the reduced phase space Dirac brackets which, together with the Hamiltonian $\mathrm{H}_{\mathrm{red}}$ in (3.35), generate the reduced phase space dynamics.

\subsection{Perturbations}

We are interested in studying the (quantum) fluctuations in the Abelian Higgs model in a situation where the scalar field develops a non-vanishing time-dependent condensate $\bar{\phi}(\eta)$. In the canonical formulation, the scalar conjugate momentum then also has a nonvanishing condensate $\bar{\pi}(\eta)$, since they both have to satisfy Hamilton's equations generated by the reduced phase space Hamiltonian (3.35),

$$
\partial_{0} \bar{\phi}=a^{2-D} \bar{\pi}, \quad \partial_{0} \bar{\pi}=-a^{D} \lambda \bar{\phi}^{3}-\xi(D-1)(D-2 \epsilon) a^{D-2} \mathcal{H}^{2} \bar{\phi} .
$$

No such condensate is assumed for the vector field, even though those have been considered in literature, most notably in the context of vector inflationary models [93-96]. The Hamiltonian encoding the dynamics of the fluctuations around such a classical configuration is 
obtained by shifting the variables of the scalar field and its conjugate momentum by their condensates,

$$
\phi(\eta, \vec{x})=\bar{\phi}(\eta)+\varphi(\eta, \vec{x}), \quad \pi(\eta, \vec{x})=\bar{\pi}(\eta)+p(\eta, \vec{x}),
$$

which then naturally organizes the reduced Hamiltonian according to the powers of the fluctuation fields,

$$
\mathrm{H}_{\text {red }}=\mathrm{H}^{(0)}+\mathrm{H}^{(2)}+\mathrm{H}^{(3)}+\mathrm{H}^{(4)}+\ldots,
$$

where $\mathrm{H}^{(0)}$ denotes the Hamiltonian for the condensate. At the linear level (quadratic Hamiltonian) the scalar and the vector field perturbations decouple,

$$
\mathrm{H}^{(2)}=\mathrm{H}_{\varphi}^{(2)}+\mathrm{H}_{A}^{(2)},
$$

where the scalar part is

$$
\mathrm{H}_{\varphi}^{(2)}=\int d^{D-1} x\left[\frac{a^{2-D}}{2} p^{2}+\frac{a^{D-2}}{2}\left(\partial_{i} \varphi\right)\left(\partial_{j} \varphi\right)+\frac{a^{D}}{2}\left(3 \lambda \bar{\phi}^{2}+\xi(D-1)(D-2 \epsilon) H^{2}\right) \varphi^{2}\right],
$$

and the vector part is

$$
\mathrm{H}_{A}^{(2)}=\int d^{D-1} x\left[\frac{a^{4-D}}{2} \Pi_{i} \Pi_{i}+\frac{a^{2-D}}{2(q \bar{\phi})^{2}}\left(\partial_{i} \Pi_{i}\right)\left(\partial_{j} \Pi_{j}\right)+\frac{a^{D-4}}{4} F_{i j} F_{i j}+\frac{a^{D-2}}{2}(q \bar{\phi})^{2} A_{i} A_{i}\right] .
$$

At higher orders the perturbations couple. For the cubic Hamiltonian we have,

$$
\mathrm{H}^{(3)}=\int d^{D-1} x\left[-\frac{a^{2-D}}{(q \bar{\phi})^{2}}\left(\frac{\varphi}{\bar{\phi}}\right)\left(\partial_{i} \Pi_{i}\right)\left(\partial_{j} \Pi_{j}\right)+a^{D-2}(q \bar{\phi})^{2}\left(\frac{\varphi}{\bar{\phi}}\right) A_{i} A_{i}+a^{D} \lambda \bar{\phi} \varphi^{3}\right],
$$

for the quartic one,

$$
\mathrm{H}^{(4)}=\int d^{D-1} x\left[\frac{3}{2} \frac{a^{2-D}}{(q \bar{\phi})^{2}}\left(\frac{\varphi}{\bar{\phi}}\right)^{2}\left(\partial_{i} \Pi_{i}\right)\left(\partial_{j} \Pi_{j}\right)+\frac{a^{D-2}}{2} q^{2} \varphi^{2} A_{i} A_{i}+\frac{a^{D}}{4} \lambda \varphi^{4}\right],
$$

and for the higher order ones,

$$
\mathrm{H}^{(n)}=\int d^{D-1} x\left[\frac{(-1)^{n}}{2}(n-1) \frac{a^{2-D}}{(q \bar{\phi})^{2}}\left(\frac{\varphi}{\bar{\phi}}\right)^{n}\left(\partial_{i} \Pi_{i}\right)\left(\partial_{j} \Pi_{j}\right)\right], \quad(n \geq 5) .
$$

It is worth noting that all orders of the reduced phase space Hamiltonians above are reproduced by the following action.

$$
\begin{aligned}
S\left[A_{\mu}, \phi\right]=\int d^{D} x \sqrt{-g} & {\left[-\frac{1}{4} g^{\mu \rho} g^{\nu \sigma} F_{\mu \nu} F_{\rho \sigma}-\frac{1}{2}(q \phi)^{2} g^{\mu \nu} A_{\mu} A_{\nu}\right.} \\
& \left.-\frac{1}{2} g^{\mu \nu}\left(\partial_{\mu} \phi\right)\left(\partial_{\nu} \phi\right)-\frac{\lambda}{4} \phi^{4}-\frac{\xi}{2} R \phi^{2}\right],
\end{aligned}
$$

after expanding it in the perturbations, $\phi=\bar{\phi}+\varphi$. The infinite tower of interactions (3.44) between the physical degrees of freedom in the canonical formulation is thus translated into a finite number of interactions in the gauge-fixed covariant formulation, at the expense of re-introducing what is now effectively an auxiliary field, $A_{0}=-a^{2-D}\left(\partial_{i} \Pi_{i}\right) /(q \phi)^{2}$. This auxiliary field is encoded in the Proca-like action above as a second-class constraint. 


\subsection{Quantization}

The quantization of the dynamics is performed in the reduced phase space formulation in the unitary gauge in three steps:

(i) All the real field perturbations are promoted to Hermitian field operators,

$$
\begin{aligned}
\varphi(x) & \rightarrow \hat{\varphi}(x), & & \hat{\varphi}^{\dagger}(x)=\hat{\varphi}(x), \\
p(x) & \rightarrow \hat{p}(x), & & \hat{p}^{\dagger}(x)=\hat{p}(x), \\
A_{i}(x) & \rightarrow \hat{A}_{i}(x), & & \hat{A}_{i}^{\dagger}(x)=\hat{A}_{i}(x), \\
\Pi_{i}(x) & \rightarrow \hat{\Pi}_{i}(x), & & \hat{\Pi}_{i}^{\dagger}(x)=\hat{\Pi}_{i}(x) .
\end{aligned}
$$

(ii) The Dirac brackets are promoted to commutators, $(\mid \cdot, \cdot) \rightarrow(-i)[\cdot, \cdot]$, and from the table 1 we see that the non-vanishing ones are,

$$
\left[\hat{\phi}(\eta, \vec{x}), \hat{p}\left(\eta, \vec{x}^{\prime}\right)\right]=i \delta^{D-1}\left(\vec{x}-\vec{x}^{\prime}\right), \quad\left[\hat{A}_{i}(\eta, \vec{x}), \hat{\Pi}_{j}\left(\eta, \vec{x}^{\prime}\right)\right]=\delta_{i j} i \delta^{D-1}\left(\vec{x}-\vec{x}^{\prime}\right) .
$$

(iii) The dynamics is given by the quantum Hamiltonian operator, which is obtained from the classical Hamiltonian (3.38) by substituting classical fields by field operators as in (3.46)-(3.49), and symmetrizing the products of non-commuting field operators if necessary. Here we contend ourselves with studying just the linear fluctuations whose dynamics is given by Hamiltonians (3.40) and (3.41), whose quantized versions are,

$$
\hat{\mathrm{H}}_{\varphi}^{(2)}=\int d^{D-1} x\left[\frac{a^{2-D}}{2} \hat{p}^{2}+\frac{a^{D-2}}{2}\left(\partial_{i} \hat{\varphi}\right)\left(\partial_{i} \hat{\varphi}\right)+\frac{a^{D}}{2}\left(3 \lambda \bar{\phi}^{2}+\xi(D-1)(D-2 \epsilon) H^{2}\right) \hat{\varphi}^{2}\right],
$$

for the scalar sector, and

$$
\hat{\mathrm{H}}_{A}^{(2)}=\int d^{D-1} x\left[\frac{a^{4-D}}{2} \hat{\Pi}_{i} \hat{\Pi}_{i}+\frac{a^{2-D}}{2(q \bar{\phi})^{2}}\left(\partial_{i} \hat{\Pi}_{i}\right)\left(\partial_{j} \hat{\Pi}_{j}\right)+\frac{a^{D-4}}{4} \hat{F}_{i j} \hat{F}_{i j}+\frac{a^{D-2}}{2}(q \bar{\phi})^{2} \hat{A}_{i} \hat{A}_{i}\right],
$$

for the vector sector. Interactions between the fields are then governed by the quantized version of the interaction Hamiltonians (3.42)-(3.44).

\section{Condensate dynamics}

In this work we consider the condensate $\bar{\phi}$ of the complex scalar to be a spectator, in the sense that it does not influence the space-time dynamics. The power-law inflation spacetime is assumed to be driven by some other field. ${ }^{11}$ The dynamics of the homogeneous and isotropic spectator condensate $\bar{\phi}$ of our Abelian Higgs model satisfies first order dynamical equations (3.36) in FLRW space-times. In power-law inflation, where $\epsilon=$ const., the two equations combine into a single second order one,

$$
\partial_{0}^{2} \bar{\phi}+(D-2) \mathcal{H} \partial_{0} \bar{\phi}+a^{2}\left[\lambda \bar{\phi}^{2}+\xi(D-1)(D-2 \epsilon) H^{2}\right] \bar{\phi}=0 .
$$

\footnotetext{
${ }^{11}$ For example, an inflaton field $\phi$ with an exponential potential $U(\phi)=U_{0} \exp \left(-\beta \phi / M_{\mathrm{P}}\right)$ yields, in its attractor regime, a power-law inflation with $\epsilon=\beta^{2} / 2$, and $\phi \propto \ln (a)$; see e.g. [10].
} 
We are interested in a particular attractor behaviour, where the condensate scales as the physical Hubble rate,

$$
\bar{\phi}=\left(\frac{\bar{\phi}_{0}}{H_{0}}\right) H=\bar{\phi}_{0} a^{-\epsilon}
$$

where the amplitude $\bar{\phi}_{0}$ is a constant. Such attractor solutions in power-law inflation have been studied already in the context of a self-interacting scalar [13], and the Yukawa model [28]. The attractor behaviour of this solution is manifested in its independence of initial conditions. The assumed scaling (4.2) yields a cubic equation for the condensate amplitude,

$$
\left[-\epsilon(D-1-2 \epsilon)+\xi(D-1)(D-2 \epsilon)+\lambda\left(\frac{\bar{\phi}_{0}}{H_{0}}\right)^{2}\right] \bar{\phi}_{0}=0,
$$

which can be seen as a dynamical generalization of the static situation in flat space, where one assumes the condensate is in a global minimum of the potential. It has a symmetric solution $\bar{\phi}_{0}=0$, and two symmetry-breaking attractor solutions,

$$
\frac{\bar{\phi}_{0}}{H_{0}}= \pm \sqrt{\frac{1}{\lambda}[\epsilon(D-1-2 \epsilon)-\xi(D-1)(D-2 \epsilon)]} .
$$

These symmetry-breaking solutions exist only if the non-minimal coupling from the action (3.1) satisfies,

$$
\xi<\frac{\epsilon(D-1-2 \epsilon)}{(D-1)(D-2 \epsilon)},
$$

and otherwise only the symmetric solution exists. In the symmetric solution the scalar condensate vanishes, which is a singular point of the unitary gauge, and in the rest of the paper we assume that the condition (4.5) is fulfilled.

The remainder of the paper is devoted to computing the dynamics and two-point functions of scalar and vector perturbations around the symmetry-breaking attractor solution (4.4) for the scalar condensate in power-law inflation.

\section{Scalar perturbations}

The dynamics of the linear scalar perturbations is encoded in the Hamiltonian (3.40), and is fully captured by the scalar two-point function,

$$
i\left[{ }^{-} \Delta^{+}\right]\left(x ; x^{\prime}\right)=\left\langle\Omega\left|\hat{\varphi}(x) \hat{\varphi}\left(x^{\prime}\right)\right| \Omega\right\rangle .
$$

Solving for the dynamics of the scalar field operators and computing the expectation value above would be straightforward. However, it becomes even simpler upon realizing that the Hamiltonian (3.40) arises from considering a massless non-minimally coupled scalar, ${ }^{12}$

$$
S_{\varphi}^{(2)}[\varphi]=\int d^{D} x \sqrt{-g}\left[-\frac{1}{2} g^{\mu \nu}\left(\partial_{\mu} \varphi\right)\left(\partial_{\nu} \varphi\right)-\frac{1}{2} \xi_{S} R \varphi^{2}\right],
$$

\footnotetext{
${ }^{12}$ This correspondence no longer holds if the scalar perturbation couples to the inflaton condensate, rather than the spectator condensate. Typically the inflaton scales as $\bar{\phi} \propto \ln (a)$, and not as $\bar{\phi} \propto H$ as the spectator does. For an example how such cases can be treated semi-analytically see e.g. ref. [30].
} 
in power-law inflationary space-time, with the effective non-minimal coupling given by,

$$
\xi_{\mathrm{S}}=\xi+\frac{3 \lambda}{(D-1)(D-2 \epsilon)}\left(\frac{\bar{\phi}_{0}}{H_{0}}\right)^{2}=-2 \xi+\frac{3 \epsilon(D-1-2 \epsilon)}{(D-1)(D-2 \epsilon)} .
$$

Therefore, the dynamics of linear scalar perturbations in our Abelian Higgs model in powerlaw inflation is equivalent to the massless non-minimally coupled scalar with the nonminimal coupling $\xi_{\mathrm{S}}$ in (5.3). The two-point functions for such a scalar with respect to the Chernikov-Tagirov-Bunch-Davies (CTBD) state [97, 98], i.e. the state that minimizes energy mode-per-mode in the asymptotic past, has been worked out in [8, 14], and we simply quote it here,

$$
i\left[{ }^{A} \Delta^{B}\right]\left(x ; x^{\prime}\right)=\left(a a^{\prime}\right)^{-\frac{(D-2) \epsilon}{2}} \times \mathcal{F}_{\nu_{\mathrm{S}}}\left(y_{A B}\right), \quad(A, B=+,-),
$$

where we define the rescaled propagator function,

$\mathcal{F}_{\lambda}(y)=\frac{\left[(1-\epsilon) H_{0}\right]^{D-2}}{(4 \pi)^{D / 2}} \frac{\Gamma\left(\frac{D-1}{2}+\lambda\right) \Gamma\left(\frac{D-1}{2}-\lambda\right)}{\Gamma\left(\frac{D}{2}\right)}{ }_{2} F_{1}\left(\left\{\frac{D-1}{2}+\lambda, \frac{D-1}{2}-\lambda\right\},\left\{\frac{D}{2}\right\}, 1-\frac{y}{4}\right)$,

whose properties are given in appendix B. The argument $y$ of the rescaled propagator function in (5.4) is the distance function defined in (2.13), and its index is

$$
\nu_{\mathrm{S}}=\sqrt{\left(\frac{D-1-\epsilon}{2(1-\epsilon)}\right)^{2}-\frac{(D-1)(D-2 \epsilon) \xi_{\mathrm{S}}}{(1-\epsilon)^{2}}} .
$$

The CTBD state in power-law inflation is known to be IR divergent for certain ranges of non-minimal couplings $[99,100]$, in which case the IR sector of the state needs to be modified and the two-point function contains additional terms. However, the IR divergence is absent from the CTBD state if the following condition on the non-minimal coupling is satisfied $[99,100]$,

$$
\xi_{\mathrm{S}}>\frac{\epsilon(D-2)[2(D-1)-D \epsilon]}{4(D-1)(D-2 \epsilon)},
$$

which here translates into a condition on the non-minimal coupling of the Abelian Higgs model,

$$
\xi<\frac{\epsilon(D-1-2 \epsilon)}{(D-1)(D-2 \epsilon)}-(D-4) \times \frac{\epsilon[2(D-1)-(D+2) \epsilon]}{8(D-1)(D-2 \epsilon)} .
$$

In $D=4$ this condition matches precisely the independent condition we found for the existence of the attractor solution for the condensate (4.5). Therefore, we conclude that the attractor condensate in power-law inflation always induces an effective non-minimal coupling $\xi_{\mathrm{S}}$ for the scalar perturbation which regulates its IR behaviour.

\section{Dynamics of vector perturbations}

The dynamics of linearized vector perturbations in the Abelian Higgs model in FLRW space-time is given by the Hamiltonian (3.41). When the background is specialized to 
power-law inflation, and the scalar condensate is in the attractor regime (4.2), this system is equivalent at the linear level to the non-minimally coupled vector field given by the action,

$$
S_{A}^{(2)}\left[A_{\mu}, \phi\right]=\int d^{D} x \sqrt{-g}\left[-\frac{1}{4} g^{\mu \rho} g^{\nu \sigma} F_{\mu \nu} F_{\rho \sigma}-\frac{1}{2} \xi_{\mathrm{V}} R g^{\mu \nu} A_{\mu} A_{\nu}\right],
$$

where the effective non-minimal coupling of the vector is given by,

$$
\xi_{\mathrm{v}}=\frac{\left(q \bar{\phi}_{0}\right)^{2}}{(D-1)(D-2 \epsilon) H_{0}^{2}}=\frac{(q \bar{\phi})^{2}}{(D-1)(D-2 \epsilon) H^{2}} .
$$

The equivalence of action (6.1) with the Hamiltonian in (3.41) can be demonstrated by deriving the canonical formulation, following the procedure of section 3.1. The difference is that here one finds two second-class constraints, which essentially amount to the first and the last equality in (3.34). In other words, the first and last equality in (3.34) that eliminates $A_{0}$ and $\Pi_{0}$ from the phase space in the unitary gauge can be used to reintroduce the eliminated fields, allowing us to write the covariant action (6.1). This equivalence is not crucial, and we make no further use of it, but it does provide a more intuitive picture and establishes a connection with the way the unitary gauge is usually treated.

In this section we solve for the dynamics of the vector field operators $\hat{A}_{i}$ and $\hat{\Pi}_{i}$ of the reduced phase space. Their dynamics is generated by the Hamiltonian operator (3.52), specialized to power-law inflation and the attractor solution for scalar condensate,

$$
\hat{\mathrm{H}}_{A}^{(2)}=\int d^{D-1} x\left[\frac{a^{4-D}}{2} \hat{\Pi}_{i} \hat{\Pi}_{i}+\frac{a^{2-D}}{2(q \bar{\phi})^{2}}\left(\partial_{i} \hat{\Pi}_{i}\right)\left(\partial_{j} \hat{\Pi}_{j}\right)+\frac{a^{D-4}}{4} \hat{F}_{i j} \hat{F}_{i j}+\frac{a^{D-2}}{2}(q \bar{\phi})^{2} \hat{A}_{i} \hat{A}_{i}\right],
$$

which generates the equations of motion,

$$
\begin{aligned}
& \partial_{0} \hat{A}_{i}=a^{4-D} \hat{\Pi}_{i}-\frac{a^{2-D}}{(q \bar{\phi})^{2}} \partial_{i}\left(\partial_{j} \hat{\Pi}_{j}\right), \\
& \partial_{0} \hat{\Pi}_{i}=a^{D-4} \partial_{j} \hat{F}_{j i}-a^{D-2}(q \bar{\phi})^{2} \hat{A}_{i} .
\end{aligned}
$$

It is convenient to decompose the fields into transverse and longitudinal sectors, and to consider the equations of motion in a spatial Fourier space. In the following sections we first perform these transformations and then proceed to solve the equations for each sector.

\subsection{Scalar-vector decomposition}

The field operators $\hat{A}_{i}$ and $\hat{\Pi}_{i}$ are decomposed into transverse and longitudinal parts,

$$
\hat{A}_{i}=\hat{A}_{i}^{\mathrm{T}}+\hat{A}_{i}^{\mathrm{L}}, \quad \hat{\Pi}_{i}=\hat{\Pi}_{i}^{\mathrm{T}}+\hat{\Pi}_{i}^{\mathrm{L}},
$$

where the longitudinal parts,

$$
\hat{A}_{i}^{\mathrm{L}}(\eta, \vec{x})=\mathbb{P}_{i j}^{\mathrm{L}}(\vec{x}) \hat{A}_{j}(\eta, \vec{x}), \quad \hat{\Pi}_{i}^{\mathrm{L}}(\eta, \vec{x})=\mathbb{P}_{i j}^{\mathrm{L}}(\vec{x}) \hat{\Pi}_{j}(\eta, \vec{x}),
$$

and the transverse ones,

$$
\hat{A}_{i}^{\mathrm{T}}(\eta, \vec{x})=\mathbb{P}_{i j}^{\mathrm{T}}(\vec{x}) \hat{A}_{j}(\eta, \vec{x}), \quad \hat{\Pi}_{i}^{\mathrm{T}}(\eta, \vec{x})=\mathbb{P}_{i j}^{\mathrm{T}}(\vec{x}) \hat{\Pi}_{j}(\eta, \vec{x}),
$$


are written with the help of the longitudinal and transverse projectors, respectively,

$$
\mathbb{P}_{i j}^{\mathrm{L}}(\vec{x})=\frac{\partial_{i} \partial_{j}}{\nabla^{2}}, \quad \mathbb{P}_{i j}^{\mathrm{T}}(\vec{x})=\delta_{i j}-\frac{\partial_{i} \partial_{j}}{\nabla^{2}}
$$

where $\nabla^{2}=\delta_{i j} \partial_{i} \partial_{j}$ is the Laplace operator. These projection operators are idempotent, $\mathbb{P}_{i j}^{\mathrm{L}}(\vec{x}) \mathbb{P}_{j k}^{\mathrm{L}}(\vec{x})=\mathbb{P}_{i k}^{\mathrm{L}}(\vec{x}), \mathbb{P}_{i j}^{\mathrm{T}}(\vec{x}) \mathbb{P}_{j k}^{\mathrm{T}}(\vec{x})=\mathbb{P}_{i k}^{\mathrm{T}}(\vec{x})$, and orthogonal, $\mathbb{P}_{i j}^{\mathrm{T}}(\vec{x}) \mathbb{P}_{j k}^{\mathrm{L}}(\vec{x})=$ $\mathbb{P}_{i j}^{\mathrm{L}}(\vec{x}) \mathbb{P}_{j k}^{\mathrm{T}}(\vec{x})=0$. Both the transverse and the longitudinal fields are Hermitian, and the non-vanishing commutators of the decomposed fields are,

$$
\begin{aligned}
{\left[\hat{A}_{i}^{\mathrm{T}}(\eta, \vec{x}), \hat{\Pi}_{j}^{\mathrm{T}}\left(\eta, \vec{x}^{\prime}\right)\right] } & =\mathbb{P}_{i j}^{\mathrm{T}}(\vec{x}) i \delta^{D-1}\left(\vec{x}-\vec{x}^{\prime}\right), \\
{\left[\hat{A}_{i}^{\mathrm{L}}(\eta, \vec{x}), \hat{\Pi}_{j}^{\mathrm{L}}\left(\eta, \vec{x}^{\prime}\right)\right] } & =\mathbb{P}_{i j}^{\mathrm{L}}(\vec{x}) i \delta^{D-1}\left(\vec{x}-\vec{x}^{\prime}\right) .
\end{aligned}
$$

The dynamics of the transverse and longitudinal sectors decouple at the linear level. This is due to the splitting of the Hamiltonian (6.3) into two disjoint parts,

$$
\hat{\mathrm{H}}_{A}^{(2)}=\hat{\mathrm{H}}^{\mathrm{T}}+\hat{\mathrm{H}}^{\mathrm{L}},
$$

where the transverse part,

$$
\hat{\mathrm{H}}^{\mathrm{T}}=\int d^{D-1} x\left[\frac{a^{4-D}}{2} \hat{\Pi}_{i}^{\mathrm{T}} \hat{\Pi}_{i}^{\mathrm{T}}+\frac{a^{D-4}}{2}\left(\partial_{i} \hat{A}_{j}^{\mathrm{T}}\right)\left(\partial_{i} \hat{A}_{j}^{\mathrm{T}}\right)+\frac{a^{D-2}}{2}(q \bar{\phi})^{2} \hat{A}_{i}^{\mathrm{T}} \hat{A}_{i}^{\mathrm{T}}\right]
$$

generates the equations of motion of the transverse sector,

$$
\begin{aligned}
\partial_{0} \hat{A}_{i}^{\mathrm{T}} & =a^{4-D} \hat{\Pi}_{i}^{\mathrm{T}}, \\
\partial_{0} \hat{\Pi}_{i}^{\mathrm{T}} & =a^{D-4} \nabla^{2} \hat{A}_{i}^{\mathrm{T}}-a^{D-2}(q \bar{\phi})^{2} \hat{A}_{i}^{\mathrm{T}},
\end{aligned}
$$

and the longitudinal part,

$$
\hat{\mathrm{H}}^{\mathrm{L}}=\int d^{D-1} x\left[\frac{a^{4-D}}{2} \hat{\Pi}_{i}^{\mathrm{L}} \hat{\Pi}_{i}^{\mathrm{L}}+\frac{a^{2-D}}{2(q \bar{\phi})^{2}}\left(\partial_{i} \hat{\Pi}_{i}^{\mathrm{L}}\right)\left(\partial_{j} \hat{\Pi}_{j}^{\mathrm{L}}\right)+\frac{a^{D-2}}{2}(q \bar{\phi})^{2} \hat{A}_{i}^{\mathrm{L}} \hat{A}_{i}^{\mathrm{L}}\right],
$$

generates the equations of motion of the longitudinal sector,

$$
\begin{aligned}
& \partial_{0} \hat{A}_{i}^{\mathrm{L}}=a^{4-D} \hat{\Pi}_{i}^{\mathrm{L}}-\frac{a^{2-D}}{(q \bar{\phi})^{2}} \nabla^{2} \hat{\Pi}_{i}^{\mathrm{L}}, \\
& \partial_{0} \hat{\Pi}_{i}^{\mathrm{L}}=-a^{D-2}(q \bar{\phi})^{2} \hat{A}_{i}^{\mathrm{L}},
\end{aligned}
$$

where we made use of $\partial_{i} \partial_{j} \hat{\Pi}_{j}^{\mathrm{L}}=\nabla^{2} \hat{\Pi}_{i}^{\mathrm{L}}$. 


\subsection{Fourier decomposition}

Homogeneity and isotropy of the FLRW space-time and the scalar condensate suggest that it is convenient to introduce the spatial Fourier decomposition of the field operators,

$$
\begin{aligned}
& \hat{A}_{i}^{\mathrm{T}}(\eta, \vec{x})=a^{-\frac{D-4}{2}} \int \frac{d^{D-1} k}{(2 \pi)^{\frac{D-1}{2}}} e^{i \vec{k} \cdot \vec{x}} \sum_{\sigma=1}^{D-2} \varepsilon_{i}(\sigma, \vec{k}) \hat{\mathcal{A}}_{\mathrm{T}, \sigma}(\eta, \vec{k}), \\
& \hat{\Pi}_{i}^{\mathrm{T}}(\eta, \vec{x})=a^{\frac{D-4}{2}} \int \frac{d^{D-1} k}{(2 \pi)^{\frac{D-1}{2}}} e^{i \vec{k} \cdot \vec{x}} \sum_{\sigma=1}^{D-2} \varepsilon_{i}(\sigma, \vec{k}) \hat{\pi}_{\mathrm{T}, \sigma}(\eta, \vec{k}), \\
& \hat{A}_{i}^{\mathrm{L}}(\eta, \vec{x})=a^{-\frac{D-4}{2}}\left(\frac{\mathcal{H}}{H_{0}}\right)^{-1} \int \frac{d^{D-1} k}{(2 \pi)^{\frac{D-1}{2}}} e^{i \vec{k} \cdot \vec{x}}\left(-\frac{i k_{i}}{k}\right) \hat{\mathcal{A}}_{\mathrm{L}}(\eta, \vec{k}), \\
& \hat{\Pi}_{i}^{\mathrm{L}}(\eta, \vec{x})=a^{\frac{D-4}{2}}\left(\frac{\mathcal{H}}{H_{0}}\right) \int \frac{d^{D-1} k}{(2 \pi)^{\frac{D-1}{2}}} e^{i \vec{k} \cdot \vec{x}}\left(-\frac{i k_{i}}{k}\right) \hat{\pi}_{\mathrm{L}}(\eta, \vec{k}),
\end{aligned}
$$

where $k=\|\vec{k}\|$, and where the $(D-2)$ transverse polarization vectors $\varepsilon_{i}(\sigma, \vec{k})$ satisfy,

$$
\begin{aligned}
k_{i} \varepsilon_{i}(\sigma, \vec{k}) & =0, & \varepsilon_{i}^{*}(\sigma, \vec{k}) & =\varepsilon_{i}(\sigma,-\vec{k}), \\
\varepsilon_{i}^{*}(\sigma, \vec{k}) \varepsilon_{i}\left(\sigma^{\prime}, \vec{k}\right) & =\delta_{\sigma \sigma^{\prime}}, & \sum_{\sigma=1}^{D-2} \varepsilon_{i}^{*}(\sigma, \vec{k}) \varepsilon_{j}(\sigma, \vec{k}) & =\delta_{i j}-\frac{k_{i} k_{j}}{k^{2}},
\end{aligned}
$$

with the summation over the repeated spatial indices implied. Note the different powers of the scale factor and the conformal Hubble rate taken out of the integrals in (6.19)-(6.22), which is done to simplify the momentum space equations of motion. The hermiticity of the field operators (3.46)-(3.49) in momentum space takes the form,

$$
\begin{aligned}
\hat{\mathcal{A}}_{\mathrm{T}, \sigma}^{\dagger}(\eta, \vec{k}) & =\hat{\mathcal{A}}_{\mathrm{T}, \sigma}(\eta,-\vec{k}), & \hat{\pi}_{\mathrm{T}, \sigma}^{\dagger}(\eta, \vec{k}) & =\hat{\pi}_{\mathrm{T}, \sigma}(\eta,-\vec{k}), \\
\hat{\mathcal{A}}_{\mathrm{L}}^{\dagger}(\eta, \vec{k}) & =\hat{\mathcal{A}}_{\mathrm{L}}(\eta,-\vec{k}), & \hat{\pi}_{\mathrm{L}}^{\dagger}(\eta, \vec{k}) & =\hat{\pi}_{\mathrm{L}}(\eta,-\vec{k}),
\end{aligned}
$$

and the non-vanishing commutators (6.11) of these momentum space field operators are,

$$
\begin{aligned}
{\left[\hat{\mathcal{A}}_{\mathrm{T}, \sigma}(\eta, \vec{k}), \hat{\pi}_{\mathrm{T}, \sigma^{\prime}}\left(\eta, \vec{k}^{\prime}\right)\right] } & =\delta_{\sigma \sigma^{\prime}} i \delta^{D-1}\left(\vec{k}+\vec{k}^{\prime}\right), \\
{\left[\hat{\mathcal{A}}_{\mathrm{L}}(\eta, \vec{k}), \hat{\pi}_{\mathrm{L}}\left(\eta, \vec{k}^{\prime}\right)\right] } & =i \delta^{D-1}\left(\vec{k}+\vec{k}^{\prime}\right) .
\end{aligned}
$$

The transverse sector Hamiltonian (6.13) expressed in momentum space reads,

$$
\hat{\mathrm{H}}^{\mathrm{T}}=\int d^{D-1} k \sum_{\sigma=1}^{D-2}\left[\frac{1}{2} \hat{\pi}_{\mathrm{T}, \sigma}^{\dagger} \hat{\pi}_{\mathrm{T}, \sigma}+\frac{1}{4}(D-4) \mathcal{H}\left\{\hat{\pi}_{\mathrm{T}, \sigma}^{\dagger}, \hat{\mathcal{A}}_{\mathrm{T}, \sigma}\right\}+\frac{1}{2}\left(k^{2}+a^{2}(q \bar{\phi})^{2}\right) \hat{\mathcal{A}}_{\mathrm{T}, \sigma}^{\dagger} \hat{\mathcal{A}}_{\mathrm{T}, \sigma}\right],
$$

where $\{\hat{\mathrm{A}}, \hat{\mathrm{B}}\}=\hat{\mathrm{A}} \hat{\mathrm{B}}+\hat{\mathrm{B}} \hat{\mathrm{A}}$ denotes the anti-commutator, and it generates the momentum space equations of motion of the transverse sector [cf. eqs. (6.14)-(6.15)],

$$
\begin{aligned}
\partial_{0} \hat{\mathcal{A}}_{\mathrm{T}, \sigma} & =\hat{\pi}_{\mathrm{T}, \sigma}+\frac{1}{2}(D-4) \mathcal{H} \hat{\mathcal{A}}_{\mathrm{T}, \sigma}, \\
\partial_{0} \hat{\pi}_{\mathrm{T}, \sigma} & =-\frac{1}{2}(D-4) \mathcal{H} \hat{\pi}_{\mathrm{T}, \sigma}-\left(k^{2}+a^{2}(q \bar{\phi})^{2}\right) \hat{\mathcal{A}}_{\mathrm{T}, \sigma} .
\end{aligned}
$$


The longitudinal sector Hamiltonian (6.16) in momentum space reads,

$$
\hat{\mathrm{H}}^{\mathrm{L}}=\int d^{D-1} k\left[\frac{1}{2\left(q \bar{\phi}_{0}\right)^{2}}\left(k^{2}+a^{2}(q \bar{\phi})^{2}\right) \hat{\pi}_{\mathrm{L}}^{\dagger} \hat{\pi}_{\mathrm{L}}+\frac{1}{4}(D-2-2 \epsilon) \mathcal{H}\left\{\hat{\pi}_{\mathrm{L}}^{\dagger}, \hat{\mathcal{A}}_{\mathrm{L}}\right\}+\frac{1}{2}\left(q \bar{\phi}_{0}\right)^{2} \hat{\mathcal{A}}_{\mathrm{L}}^{\dagger} \hat{\mathcal{A}}_{\mathrm{L}}\right],
$$

and generates the momentum space equations of motions of the longitudinal sector [cf. eqs. (6.17)-(6.18)],

$$
\begin{aligned}
\partial_{0} \hat{\mathcal{A}}_{\mathrm{L}} & =\frac{1}{\left(q \bar{\phi}_{0}\right)^{2}}\left(k^{2}+a^{2}(q \bar{\phi})^{2}\right) \hat{\pi}_{\mathrm{L}}+\frac{1}{2}(D-2-2 \epsilon) \mathcal{H} \hat{\mathcal{A}}_{\mathrm{L}} \\
\partial_{0} \hat{\pi}_{\mathrm{L}} & =-\frac{1}{2}(D-2-2 \epsilon) \mathcal{H} \hat{\pi}_{\mathrm{L}}-\left(q \bar{\phi}_{0}\right)^{2} \hat{\mathcal{A}}_{\mathrm{L}}
\end{aligned}
$$

Note that the momentum space Hamiltonians (6.28) and (6.31) are not merely field transformations of the position space Hamiltonians (6.13) and (6.16), but contain additional parts originating from the explicit time dependence of the Fourier transforms (6.19)-(6.22).

\subsection{Dynamics of the transverse sector}

The two first-order equations (6.29) and (6.30) for the transverse fields can be combined into a second order equation for the transverse vector potential, whose solution then determines the transverse canonical momentum,

$$
\begin{aligned}
{\left[\partial_{0}^{2}+k^{2}-\left(\nu^{2}-\frac{1}{4}\right)(1-\epsilon)^{2} \mathcal{H}^{2}\right] \hat{\mathcal{A}}_{\mathrm{T}, \sigma} } & =0 \\
\hat{\pi}_{\mathrm{T}, \sigma} & =\left[\partial_{0}-\frac{1}{2}(D-4) \mathcal{H}\right] \hat{\mathcal{A}}_{\mathrm{T}, \sigma},
\end{aligned}
$$

where we have introduced the index for the vector field mode functions,

$$
\nu=\sqrt{\left(\frac{D-3-\epsilon}{2(1-\epsilon)}\right)^{2}-\frac{\left(q \bar{\phi}_{0}\right)^{2}}{(1-\epsilon)^{2} H_{0}^{2}}},
$$

which is either purely real and positive-semidefinite, or purely imaginary, depending on the value of the condensate parameter $\left(q \bar{\phi}_{0}\right)^{2} / H_{0}^{2}$. Equation (6.34) is of the form typical for scalar mode functions in power-law inflation. Its general solutions and their properties are given in appendix A. Here we give the solutions as,

$$
\begin{aligned}
\hat{\mathcal{A}}_{\mathrm{T}, \sigma}(\eta, \vec{k})= & U_{\nu}(\eta, k) \hat{b}_{\mathrm{T}, \sigma}(\vec{k})+U_{\nu}^{*}(\eta, k) \hat{b}_{\mathrm{T}, \sigma}^{\dagger}(-\vec{k}), \\
\hat{\pi}_{\mathrm{T}, \sigma}(\eta, \vec{k})= & {\left[\partial_{0}-\frac{1}{2}(D-4) \mathcal{H}\right] U_{\nu}(\eta, k) \hat{b}_{\mathrm{T}, \sigma}(\vec{k}) } \\
& +\left[\partial_{0}-\frac{1}{2}(D-4) \mathcal{H}\right] U_{\nu}^{*}(\eta, k) \hat{b}_{\mathrm{T}, \sigma}^{\dagger}(-\vec{k}),
\end{aligned}
$$

where the mode function $U_{\nu}$ given in (A.3) is,

$$
U_{\nu}(\eta, k)=e^{\frac{i \pi}{4}(2 \nu+1)} e^{\frac{-i k}{(1-\epsilon) \mathcal{H}_{0}}} \sqrt{\frac{\pi}{4(1-\epsilon) \mathcal{H}}} H_{\nu}^{(1)}\left(\frac{k}{(1-\epsilon) \mathcal{H}}\right)
$$


and where $H_{\nu}^{(1)}$ is the Hankel function of the first kind. The time-independent operators $\hat{b}_{\mathrm{T}, \sigma}^{\dagger}(\vec{k})$ and $\hat{b}_{\mathrm{T}, \sigma}(\vec{k})$ are introduced as initial conditions for field operators, and are chosen such that they satisfy the commutation relations for creation and annihilation operators,

$$
\left[\hat{b}_{\mathrm{T}, \sigma}(\vec{k}), \hat{b}_{\mathrm{T}, \sigma^{\prime}}^{\dagger}\left(\vec{k}^{\prime}\right)\right]=\delta_{\sigma \sigma^{\prime}} \delta^{D-1}\left(\vec{k}-\vec{k}^{\prime}\right),
$$

owing to the fact that the mode functions are normalized to the Wronskian given in (A.4),

$$
U_{\nu}(\eta, k) \partial_{0} U_{\nu}^{*}(\eta, k)-U_{\nu}^{*}(\eta, k) \partial_{0} U_{\nu}(\eta, k)=i
$$

and that the transverse field operators commute as in (6.26). A more detailed discussion of the meaning of these operators is postponed until section 7.2. When expressed in terms of the creation and annihilation operators, the transverse sector Hamiltonian (6.28) reads,

$$
\begin{aligned}
\hat{\mathrm{H}}_{\mathrm{T}}=\int d^{D-1} k & \sum_{\sigma=1}^{D-2} \frac{1}{2}\left\{\left[\left|\partial_{0} U_{\nu}\right|^{2}+\left(k^{2}+a^{2}(q \bar{\phi})^{2}-\frac{1}{4}(D-4) \mathcal{H}^{2}\right)\left|U_{\nu}\right|^{2}\right]\left\{\hat{b}_{\mathrm{T}, \sigma}(\vec{k}), \hat{b}_{\mathrm{T}, \sigma}(\vec{k})\right\}\right. \\
& +\left[\left(\partial_{0} U_{\nu}\right)^{2}+\left(k^{2}+a^{2}(q \bar{\phi})^{2}-\frac{1}{4}(D-4) \mathcal{H}^{2}\right)\left(U_{\nu}\right)^{2}\right] \hat{b}_{\mathrm{T}, \sigma}(\vec{k}) \hat{b}_{\mathrm{T}, \sigma}(-\vec{k}) \\
& \left.+\left[\left(\partial_{0} U_{\nu}^{*}\right)^{2}+\left(k^{2}+a^{2}(q \bar{\phi})^{2}-\frac{1}{4}(D-4) \mathcal{H}^{2}\right)\left(U_{\nu}^{*}\right)^{2}\right] \hat{b}_{\mathrm{T}, \sigma}^{\dagger}(\vec{k}) \hat{b}_{\mathrm{T}, \sigma}^{\dagger}(-\vec{k})\right\}
\end{aligned}
$$

The second and third line contain operators that induce a squeezing of the state [101], which are absent in flat space.

\subsection{Dynamics of the longitudinal sector}

The two first order equations (6.32) and (6.33) for the longitudinal fields are combined into a single second order equation for the longitudinal canonical momentum with the longitudinal vector potential being determined by its solution,

$$
\begin{aligned}
{\left[\partial_{0}^{2}+k^{2}-\left(\nu^{2}-\frac{1}{4}\right)(1-\epsilon)^{2} \mathcal{H}^{2}\right] \hat{\pi}_{\mathrm{L}} } & =0, \\
\hat{\mathcal{A}}_{\mathrm{L}} & =-\frac{1}{\left(q \bar{\phi}_{0}\right)^{2}}\left[\partial_{0}+\frac{1}{2}(D-2-2 \epsilon) \mathcal{H}\right] \hat{\pi}_{\mathrm{L}},
\end{aligned}
$$

where the index $\nu$ was already defined in (6.36). Equation (6.43) is the same as equation (6.34) we had solved in the preceding section, so we have for the solutions,

$$
\begin{aligned}
\hat{\pi}_{\mathrm{L}}(\eta, \vec{k}) & =\left(q \bar{\phi}_{0}\right) U_{\nu}(\eta, k) \hat{b}_{\mathrm{L}}(\vec{k})+\left(q \bar{\phi}_{0}\right) U_{\nu}^{*}(\eta, k) \hat{b}_{\mathrm{L}}^{\dagger}(-\vec{k}) \\
\hat{\mathcal{A}}_{\mathrm{L}}(\eta, \vec{k}) & =-\frac{1}{\left(q \bar{\phi}_{0}\right)}\left[\partial_{0}+\frac{1}{2}(D-2-2 \epsilon) \mathcal{H}\right]\left[U_{\nu}(\eta, k) \hat{b}_{\mathrm{L}}(\vec{k})+U_{\nu}^{*}(\eta, k) \hat{b}_{\mathrm{L}}^{\dagger}(-\vec{k})\right] .
\end{aligned}
$$

The normalization in the solutions above was chosen such that the time-independent operators $\hat{b}_{\mathrm{L}}^{\dagger}(\vec{k})$ and $\hat{b}_{\mathrm{L}}(\vec{k})$ satisfy the commutation relations for creation/annihilation operators,

$$
\left[\hat{b}_{\mathrm{L}}(\vec{k}), \hat{b}_{\mathrm{L}}^{\dagger}\left(\vec{k}^{\prime}\right)\right]=\delta^{D-1}\left(\vec{k}-\vec{k}^{\prime}\right)
$$


The Hamiltonian operator of the longitudinal sector (6.31) expressed in terms of the creation and annihilation operators is,

$$
\begin{array}{rl}
\hat{\mathrm{H}}_{\mathrm{L}}=\int d^{D-1} & k \frac{1}{2}\left\{\left[\left|\partial_{0} U_{\nu}\right|^{2}+\left(k^{2}+a^{2}(q \bar{\phi})^{2}-\frac{1}{4}(D-2-2 \epsilon)^{2} \mathcal{H}^{2}\right)\left|U_{\nu}\right|^{2}\right]\left\{\hat{b}_{\mathrm{L}}^{\dagger}(\vec{k}), \hat{b}_{\mathrm{L}}(\vec{k})\right\}\right. \\
+ & {\left[\left(\partial_{0} U_{\nu}\right)^{2}+\left(k^{2}+a^{2}(q \bar{\phi})^{2}-\frac{1}{4}(D-2-2 \epsilon)^{2} \mathcal{H}^{2}\right)\left(U_{\nu}\right)^{2}\right] \hat{b}_{\mathrm{L}}(\vec{k}) \hat{b}_{\mathrm{L}}(-\vec{k})} \\
+ & \left.\left[\left(\partial_{0} U_{\nu}^{*}\right)^{2}+\left(k^{2}+a^{2}(q \bar{\phi})^{2}-\frac{1}{4}(D-2-2 \epsilon)^{2} \mathcal{H}^{2}\right)\left(U_{\nu}^{*}\right)^{2}\right] \hat{b}_{\mathrm{L}}^{\dagger}(\vec{k}) \hat{b}_{\mathrm{L}}^{\dagger}(-\vec{k})\right\}
\end{array}
$$

\section{$7 \quad$ Vector field two-point functions}

The linearized vector perturbations are assumed to be in some Gaussian state $|\Omega\rangle$, in which case their dynamics is fully captured by the Wightman two-point function,

$$
\left\langle\Omega\left|\hat{A}_{\mu}(x) \hat{A}_{\nu}\left(x^{\prime}\right)\right| \Omega\right\rangle
$$

In this section we first properly define various two-point functions, and derive some of their properties independent of the choice of the state. We then proceed to construct the quantum state of interest, and to compute the associated two-point functions. The section closes by discussing various limits of the computed two-point functions.

\subsection{Equations of motion for two-point functions}

The meaning of the definition of the covariant Wightman two-point function given in (7.1) at the beginning of this section is not immediately clear, as it contains field operators corresponding to the temporal component of the vector potential, which we have explicitly eliminated in the gauge-fixing procedure in section 3.1. Even though this field component is redundant in the unitary gauge, there is a lot to be gained by adopting a covariant representation. To this end the temporal component $\hat{A}_{0}$ of the vector potential can be restored, not as an independent field, but rather through the very expression (3.34) used to eliminate it in the first place,

$$
\hat{A}_{0}=-\frac{a^{2-D}}{(q \bar{\phi})^{2}}\left(\partial_{i} \hat{\Pi}_{i}^{\mathrm{L}}\right)
$$

It follows from this definition and the equation of motion (6.18) that the covariant vector potential operator is transverse in the following sense,

$$
g^{\mu \nu} \nabla_{\mu}\left[(q \bar{\phi})^{2} \hat{A}_{\nu}\right]=0 .
$$

Restoring the temporal component allows us to write the operator equations of motion (6.4)-(6.5) in a more familiar form, as covariant second order equations,

$$
\mathcal{D}_{\mu}{ }^{\nu} \hat{A}_{\nu}(x)=0, \quad \mathcal{D}_{\mu \nu}=g_{\mu \nu} \square-\nabla_{\nu} \nabla_{\mu}-g_{\mu \nu}(q \bar{\phi})^{2} .
$$


This equation is written in a covariant form, but strictly speaking we have derived it only for FLRW backgrounds with homogeneous and isotropic condensates. However, it is reasonable to posit that it holds in general space-times and for general condensates. In the remainder of this section, whenever a covariant expression appears, strictly speaking it should be considered as evaluated in a FLRW space-time. Even though we have restored covariance to the equations of motion, the commutators between the covariant vector field components do not take on a covariant appearance,

$$
\begin{aligned}
{\left[\hat{A}_{0}(\eta, \vec{x}), \hat{A}_{i}\left(\eta, \vec{x}^{\prime}\right)\right] } & =\frac{a^{2-D}}{(q \bar{\phi})^{2}} \partial_{i} i \delta^{D-1}\left(\vec{x}-\vec{x}^{\prime}\right), \\
{\left[\hat{A}_{0}(\eta, \vec{x}), \partial_{0} \hat{A}_{0}\left(\eta, \vec{x}^{\prime}\right)\right] } & =-\frac{a^{2-D}}{(q \bar{\phi})^{2}} \nabla^{2} i \delta^{D-1}\left(\vec{x}-\vec{x}^{\prime}\right), \\
{\left[\hat{A}_{i}(\eta, \vec{x}), \partial_{0} \hat{A}_{0}\left(\eta, \vec{x}^{\prime}\right)\right] } & =\partial_{0}\left(\frac{a^{2-D}}{(q \bar{\phi})^{2}}\right) \partial_{i} i \delta^{D-1}\left(\vec{x}-\vec{x}^{\prime}\right), \\
{\left[\hat{A}_{i}(\eta, \vec{x}), \partial_{0} \hat{A}_{j}\left(\eta, \vec{x}^{\prime}\right)\right] } & =a^{2-D}\left(g_{i j}-\frac{\partial_{i} \partial_{j}}{(q \bar{\phi})^{2}}\right) i \delta^{D-1}\left(\vec{x}-\vec{x}^{\prime}\right) .
\end{aligned}
$$

These were computed using the fundamental commutators (3.50) of the reduced phase space, the operator equations of motion (6.4)-(6.5), and the definition (7.2) for the temporal component of the vector potential.

In what follows we define different two-point functions that are needed for the time dependent perturbation theory in the Schwinger-Keldysh (in-in, closed-time-path) formalism. The positive- and the negative-frequency Wightman functions are respectively defined by, ${ }^{13}$

$$
\begin{aligned}
& i\left[{ }_{\mu}^{-} \Delta_{\nu}^{+}\right]\left(x ; x^{\prime}\right)=\left\langle\Omega\left|\hat{A}_{\mu}(x) \hat{A}_{\nu}\left(x^{\prime}\right)\right| \Omega\right\rangle, \\
& i\left[{ }_{\mu}^{+} \Delta_{\nu}^{-}\right]\left(x ; x^{\prime}\right)=\left\langle\Omega\left|\hat{A}_{\nu}\left(x^{\prime}\right) \hat{A}_{\mu}(x)\right| \Omega\right\rangle=\left\{i\left[{ }_{\mu}^{-} \Delta_{\nu}^{+}\right]\left(x ; x^{\prime}\right)\right\}^{*},
\end{aligned}
$$

and the Feynman (time-ordered) and the Dyson (anti-time-ordered) propagators are,

$$
\begin{aligned}
i\left[{ }_{\mu}^{+} \Delta_{\nu}^{+}\right]\left(x ; x^{\prime}\right) & =\left\langle\Omega\left|\mathcal{T} \hat{A}_{\mu}(x) \hat{A}_{\nu}\left(x^{\prime}\right)\right| \Omega\right\rangle \\
& =\theta\left(\eta-\eta^{\prime}\right) i\left[{ }_{\mu}^{-} \Delta_{\nu}^{+}\right]\left(x ; x^{\prime}\right)+\theta\left(\eta^{\prime}-\eta\right) i\left[{ }_{\mu}^{+} \Delta_{\nu}^{-}\right]\left(x ; x^{\prime}\right), \\
i\left[{ }_{\mu}^{-} \Delta_{\nu}^{-}\right]\left(x ; x^{\prime}\right) & =\left\langle\Omega\left|\widetilde{\mathcal{T}} \hat{A}_{\nu}\left(x^{\prime}\right) \hat{A}_{\mu}(x)\right| \Omega\right\rangle=\left\{i\left[{ }_{\mu}^{+} \Delta_{\nu}^{+}\right]\left(x ; x^{\prime}\right)\right\}^{*},
\end{aligned}
$$

The operators $\mathcal{T}$ and $\widetilde{\mathcal{T}}$ denote time ordering and anti-time-ordering, respectively, of the field operators in the product.

The transversality (7.3) of the vector potential field operator implies some properties of the two-point functions which are independent of the state. Namely, the Wightman function is transverse on both legs,

$$
\nabla^{\mu}\left\{(q \bar{\phi})^{2} i\left[{ }_{\mu}^{-} \Delta_{\nu}^{+}\right]\left(x ; x^{\prime}\right)\right\}=0, \quad \nabla^{\prime \sigma}\left\{\left(q \bar{\phi}^{\prime}\right)^{2} i\left[{ }_{\mu}^{-} \Delta_{\nu}^{+}\right]\left(x ; x^{\prime}\right)\right\}=0,
$$

\footnotetext{
${ }^{13}$ The \pm labeling conventions for two-point functions are adopted from the Schwinger-Keldysh formalism, see e.g. [91, 92].
} 
but the Feynman propagator is not,

$$
\begin{aligned}
\nabla^{\mu}\left\{(q \bar{\phi})^{2} i\left[{ }_{\mu}^{+} \Delta_{\nu}^{+}\right]\left(x ; x^{\prime}\right)\right\} & =\delta_{\nu}^{i} \partial_{i}^{\prime} \frac{i \delta^{D}\left(x-x^{\prime}\right)}{\sqrt{-g}}, \\
\nabla^{\prime \nu}\left\{\left(q \bar{\phi}^{\prime}\right)^{2} i\left[{ }_{\mu}^{+} \Delta_{\nu}^{+}\right]\left(x ; x^{\prime}\right)\right\} & =\delta_{\mu}^{i} \partial_{i} \frac{i \delta^{D}\left(x-x^{\prime}\right)}{\sqrt{-g}} .
\end{aligned}
$$

The non-transversality of the latter one follows from its definition (7.11) that includes a time-ordered product, and from the non-vanishing commutator (7.5). The non-covariance of these relations might seem disconcerting, even though they are dictated by the rules of canonical quantization. However, there seems to be no a priori reason to expect that a time-ordered product, which singles out the time coordinate, must respect covariance.

The equations of motion for the two-point functions follow from the equation of motion for the vector potential field operator (7.4). The Wightman function satisfies homogeneous equations on both legs,

$$
\mathcal{D}_{\mu}{ }^{\rho} i\left[{ }_{\rho}^{-} \Delta_{\nu}^{+}\right]\left(x ; x^{\prime}\right)=0, \quad \mathcal{D}_{\nu}^{\prime \sigma} i\left[{ }_{\mu}{ }^{-}{ }_{\sigma}^{+}\right]\left(x ; x^{\prime}\right)=0,
$$

while the Feynman propagator satisfies inhomogeneous ones,

$$
\begin{aligned}
\mathcal{D}_{\mu}{ }^{\rho} i\left[{ }_{\rho}^{+} \Delta_{\nu}^{+}\right]\left(x ; x^{\prime}\right)= & \left(g_{\mu \nu}+a^{2} \delta_{\mu}^{0} \delta_{\nu}^{0}\right) \frac{i \delta^{D}\left(x-x^{\prime}\right)}{\sqrt{-g}} \\
& -\left(\delta_{\mu}^{0} \delta_{\nu}^{0} \nabla^{2}+\delta_{\mu}^{i} \delta_{\nu}^{0}\left[\partial_{0}+(D-2) \mathcal{H}\right] \partial_{i}\right)\left[\frac{1}{(q \bar{\phi})^{2}} \frac{i \delta^{D}\left(x-x^{\prime}\right)}{\sqrt{-g}}\right], \\
\mathcal{D}_{\nu}^{\prime \sigma} i\left[{ }_{\mu}^{+} \Delta_{\sigma}^{+}\right]\left(x ; x^{\prime}\right)= & \left(g_{\mu \nu}+a^{2} \delta_{\mu}^{0} \delta_{\nu}^{0}\right) \frac{i \delta^{D}\left(x-x^{\prime}\right)}{\sqrt{-g}} \\
& -\left(\delta_{\mu}^{0} \delta_{\nu}^{0} \nabla^{\prime 2}+\delta_{\mu}^{i} \delta_{\nu}^{0}\left[\partial_{0}^{\prime}+(D-2) \mathcal{H}^{\prime}\right] \partial_{i}^{\prime}\right)\left[\frac{1}{(q \bar{\phi})^{2}} \frac{i \delta^{D}\left(x-x^{\prime}\right)}{\sqrt{-g}}\right],
\end{aligned}
$$

where the sources originate from the time-ordering in the definition, and from the nonvanishing commutators (7.5)-(7.8). The non-covariant sources for the Feynman propagator might seem even more disconcerting than the non-transversality relations (7.14)-(7.15), but we stress again that this is dictated by the rules of canonical quantization, and is in fact well known in the Proca model in flat space [70]. There is an important observation to be made here, that the Feynman propagator is not the Green's function of the theory.

A Green's function ought to satisfy equations of motion with simpler covariant sources. Such a function can be constructed from the Feynman propagator by adding to it an additional local piece, ${ }^{14}$

$$
i\left[{ }_{\mu}^{+} G_{\nu}^{+}\right]\left(x ; x^{\prime}\right) \equiv i\left[{ }_{\mu}^{+} \Delta_{\nu}^{+}\right]\left(x ; x^{\prime}\right)+\frac{a^{2} \delta_{\mu}^{0} \delta_{\nu}^{0}}{(q \bar{\phi})^{2}} \frac{i \delta^{D}\left(x-x^{\prime}\right)}{\sqrt{-g}} .
$$

We emphasize that this is not a modification of the definition of the time ordering operation. There is nothing wrong with the Feynman propagator defined as an expectation value of

\footnotetext{
${ }^{14}$ Since this Green's function is constructed from the Feynman propagator we label it with the same $(++)$ Schwinger-Keldysh polarities.
} 
the time-ordered product. It just happens that the Feynman propagator is not a Green's function for the differential operator $\mathcal{D}_{\mu}{ }^{\nu}$. The Green's function in (7.19) is not transverse, but in a covariant manner,

$$
\begin{aligned}
\nabla^{\mu}\left\{(q \bar{\phi})^{2} i\left[{ }_{\mu}^{+} G_{\nu}^{+}\right]\left(x ; x^{\prime}\right)\right\} & =\partial_{\nu}^{\prime} \frac{i \delta^{D}\left(x-x^{\prime}\right)}{\sqrt{-g}}, \\
\nabla^{\prime \nu}\left\{\left(q \bar{\phi}^{\prime}\right)^{2} i\left[{ }_{\mu}^{+} G_{\nu}^{+}\right]\left(x ; x^{\prime}\right)\right\} & =\partial_{\mu} \frac{i \delta^{D}\left(x-x^{\prime}\right)}{\sqrt{-g}} .
\end{aligned}
$$

and the equations of motion it satisfies are covariant as well,

$$
\mathcal{D}_{\mu}{ }^{\rho} i\left[{ }_{\rho}^{+} G_{\nu}^{+}\right]\left(x ; x^{\prime}\right)=g_{\mu \nu} \frac{i \delta^{D}\left(x-x^{\prime}\right)}{\sqrt{-g}}, \quad \mathcal{D}_{\nu}^{\prime \sigma} i\left[{ }_{\mu}^{+} G_{\sigma}^{+}\right]\left(x ; x^{\prime}\right)=g_{\mu \nu} \frac{i \delta^{D}\left(x-x^{\prime}\right)}{\sqrt{-g}} .
$$

It is worth pointing out that the retarded Green's function is constructed from the Wightman function (7.10), and the (++) Green's function in (7.19),

$$
i\left[{ }_{\mu} G_{\nu}^{\mathrm{R}}\right]\left(x ; x^{\prime}\right)=i\left[{ }_{\mu}^{+} G_{\nu}^{+}\right]\left(x ; x^{\prime}\right)-i\left[{ }_{\mu}^{+} \Delta_{\nu}^{-}\right]\left(x ; x^{\prime}\right) .
$$

and not from the Feynman propagator (7.11). It is this retarded Green's function that describes causal propagation of (quantum) field perturbations. This distinction between the Feynman propagator and the Green's function in the unitary gauge of scalar electrodynamics (and in Proca theory), that results from canonical quantization, is well established in flat space [57, 70], and has also been noted in de Sitter space [102].

The distinction between the Feynman propagator and the $(++)$ Green's function bares implications for the Feynman rules of perturbation theory, since in the diagrammatic expansion there is a difference between the vector line descending from an actual expectation value of a product of operators, and a line descending from inverting the kinetic operator in the Yang-Feldman equation. If one insists on using only the Green's function or only the Feynman propagator in perturbation theory in the unitary gauge, then the local terms by which they differ must be taken into account. This is the reason for the additional counterterms that have to be added to the effective action in the unitary gauge to restore renormalizability [57-59]. If the distinction between the Feynman propagator and the Green's function is maintained, the additional counterterms should not be necessary. One should use the Yang-Feldman equation [70, 71] to uniquely determine which two-point function appears in the perturbative expansion.

\subsection{Choice of the state}

The two-point functions depend on the physical state of the system $|\Omega\rangle$, which needs to be specified before computing them. The evolution of vector perturbations at leading order is governed by linear equations, and therefore it is natural to assume their quantum state to be Gaussian, since the evolution does not change this property. A pure Gaussian state $|\Omega\rangle$ with a vanishing condensate is in general defined as being annihilated by linear combinations of creation and annihilation operators of the transverse and the longitudinal sectors, for each mode $\vec{k}$ and helicity $\sigma$,

$$
\left[\alpha_{\mathrm{T}, \sigma}(k) \hat{b}_{\mathrm{T}, \sigma}(\vec{k})+\beta_{\mathrm{T}, \sigma}(k) \hat{b}_{\mathrm{T}, \sigma}^{\dagger}(\vec{k})\right]|\Omega\rangle=0, \quad\left[\alpha_{\mathrm{L}}(k) \hat{b}_{\mathrm{L}}(\vec{k})+\beta_{\mathrm{L}}(k) \hat{b}_{\mathrm{L}}^{\dagger}(\vec{k})\right]|\Omega\rangle=0,
$$


where we have assumed the operators from different sectors and of different helicities do not mix, which is preserved by the dynamics on cosmological backgrounds. The coefficients in the two conditions above can be seen to be the Bogolyubov coefficients, which have to satisfy,

$$
\left|\alpha_{\mathrm{T}, \sigma}(k)\right|^{2}-\left|\beta_{\mathrm{T}, \sigma}(k)\right|^{2}=1, \quad\left|\alpha_{\mathrm{L}}(k)\right|^{2}-\left|\beta_{\mathrm{L}}(k)\right|^{2}=1,
$$

on the account of commutators of creation/annihilation operators. In general they depend on the momentum vector, but in the spirit of homogeneity and isotropy of the cosmological setting we assume they depend just on the modulus of the momentum, $k=\|\vec{k}\|$. Specifying Bogolyubov coefficients completely determines the Gaussian state. ${ }^{15}$

In accelerating cosmological space-times, such as power-law inflation, the physically distinct state is the one which minimizes the energy mode-per-mode in the asymptotic past, which is the analogue of the Chernikov-Tagirov-Bunch-Davies (CTBD) state in de Sitter space [97, 98]. Determining the Bogolyubov coefficients corresponding to this condition is accomplished by taking the expectation value of the transverse and longitudinal sector Hamiltonians (6.42) and (6.48), and minimizing their values mode-by-mode (as described in detail in Ch. 6 of ref. [103]). The result of this tedious exercise is simply $\alpha_{\mathrm{T}, \sigma}=\alpha_{\mathrm{L}}=1$ and $\beta_{\mathrm{T}, \sigma}=\beta_{\mathrm{L}}=0$, so that the CTBD state we consider here is defined by,

$$
\hat{b}_{\mathrm{T}, \sigma}(\vec{k})|\Omega\rangle=0, \quad \hat{b}_{\mathrm{L}}(\vec{k})|\Omega\rangle=0, \quad \forall \vec{k}, \sigma .
$$

These conditions uniquely define the state, and the solutions for the vector potential field operators (6.37)-(6.38) and (6.45)-(6.46) now completely determine the Wightman function (7.1) given at the beginning of the section, and other related two-point functions.

\subsection{Two-point functions as mode sums}

In this section we make use of explicit solutions for the field operators found in section 6 to compute the covariant Wightman two-point function, introduced in (7.9), for the state defined in section 7.2. The Feynman propagator then follows from its definition (7.11) in terms of the Wightman functions, and the Green's function from (7.19). As the temporal and longitudinal components of the vector potential are expressed in terms of the longitudinal momentum according to (6.18) and (7.2),

$$
\hat{A}_{0}=-\frac{a^{2-D}}{(q \bar{\phi})^{2}}\left(\partial_{i} \Pi_{i}^{\mathrm{L}}\right), \quad \hat{A}_{i}^{\mathrm{L}}=-\frac{a^{2-D}}{(q \bar{\phi})^{2}}\left(\partial_{0} \Pi_{i}^{\mathrm{L}}\right)
$$

for the purpose of computing the Wightman function it is enough to compute the two-point functions of the transverse vector potential, and the longitudinal canonical momentum. Using the Fourier decompositions (6.19) and (6.22) of the said field operators, and their solutions (6.37) and (6.45), the required two-point functions are expressed as,

$$
\begin{aligned}
\left\langle\Omega\left|\hat{A}_{i}^{\mathrm{T}}(\eta, \vec{x}) \hat{A}_{j}^{\mathrm{T}}\left(\eta^{\prime}, \vec{x}^{\prime}\right)\right| \Omega\right\rangle & =\left(a a^{\prime}\right) \mathbb{P}_{i j}^{\mathrm{T}}(\vec{x}) i\left[{ }^{-} \Delta^{+}\right]_{\nu}\left(x ; x^{\prime}\right), \\
\left\langle\Omega\left|\hat{\Pi}_{i}^{\mathrm{L}}(\eta, \vec{x}) \hat{\Pi}_{j}^{\mathrm{L}}\left(\eta^{\prime}, \vec{x}^{\prime}\right)\right| \Omega\right\rangle & =\left(a a^{\prime}\right)^{D-2}(q \bar{\phi})\left(q \bar{\phi}^{\prime}\right) \mathbb{P}_{i j}^{\mathrm{L}}(\vec{x}) i\left[{ }^{-} \Delta^{+}\right]_{\nu}\left(x ; x^{\prime}\right),
\end{aligned}
$$

\footnotetext{
${ }^{15}$ Here we only work with pure Gaussian states. The most general Gaussian states are mixed states; see e.g. ref. [92] for how to define general mixed Gaussian states.
} 
where only a single integral over modes appears,

$$
i\left[-\Delta^{+}\right]_{\nu}\left(x ; x^{\prime}\right)=\left(a a^{\prime}\right)^{-\frac{D-2}{2}} \int \frac{d^{D-1} k}{(2 \pi)^{\frac{D-1}{2}}} e^{i \vec{k} \cdot \vec{x}} U_{\nu}(\eta, k) U_{\nu}^{*}\left(\eta^{\prime}, k\right),
$$

and where the parameter $\nu$ is defined in (6.36). As long as $\nu^{2}<(D-1)^{2} / 4$, this integral is IR finite and can be recognized as the Wightman function for a scalar field in spaces of constant $\epsilon$ [14], with an effective non-minimal coupling $\xi_{\mathrm{eff}}=\frac{(D-1-\epsilon)^{2}-4(1-\epsilon)^{2} \nu^{2}}{4(D-1)(D-2 \epsilon)}$. The inequality condition in our case reads,

$$
\frac{\left(q \bar{\phi}_{0}\right)^{2}}{H_{0}^{2}}>-(1-\epsilon)^{2}\left(1-\frac{(D-4) \epsilon}{2(1-\epsilon)}\right)\left(2+\frac{(D-4)(2-\epsilon)}{2(1-\epsilon)}\right) .
$$

and is always satisfied in $D=4$, since the scalar condensate parameter $\left(q \bar{\phi}_{0}\right)^{2} / H_{0}^{2}$ is always positive, and the right hand side always negative. The scalar condensate thus regulates the infrared of the vector perturbations as well as the scalar ones. We have already encountered the integral over modes in (7.29) in section 5, and its solution is

$$
i\left[{ }^{-} \Delta^{+}\right]_{\nu}\left(x ; x^{\prime}\right)=\left(a a^{\prime}\right)^{-\frac{(D-2) \epsilon}{2}} \times \mathcal{F}_{\nu}\left(y_{-+}\right)
$$

where the rescaled propagator function was defined in (5.5), and $y_{-+}$was introduced in (2.13). The way to evaluate the integral in (7.29) and to obtain the above solution is described in considerable detail in ref. [8], and therefore we do not repeat it here. The two-point functions from (7.27) and (7.28) are now expressed as,

$$
\begin{aligned}
& \left\langle\Omega\left|\hat{A}_{i}^{\mathrm{T}}(\eta, \vec{x}) \hat{A}_{j}^{\mathrm{T}}\left(\eta^{\prime}, \vec{x}^{\prime}\right)\right| \Omega\right\rangle=\left(a a^{\prime}\right)^{1-\frac{(D-2) \epsilon}{2}} \mathbb{P}_{i j}^{\mathrm{T}}(\vec{x}) \mathcal{F}_{\nu}\left(y_{-+}\right) \\
& \left\langle\Omega\left|\hat{\Pi}_{i}^{\mathrm{L}}(\eta, \vec{x}) \hat{\Pi}_{i}^{\mathrm{L}}\left(\eta^{\prime}, \vec{x}^{\prime}\right)\right| \Omega\right\rangle=\left(a a^{\prime}\right)^{D-2-\frac{D}{2} \epsilon}\left(q \bar{\phi}_{0}\right)^{2} \mathbb{P}_{i j}^{\mathrm{L}}(\vec{x}) \mathcal{F}_{\nu}\left(y_{-+}\right) .
\end{aligned}
$$

Computing the covariant Wightman function of the vector potential field is now a straightforward matter of writing out its components according to (7.26) and plugging in the two-point functions (7.32)-(7.33),

$$
\begin{aligned}
i\left[{ }_{0} \Delta_{0}^{+}\right]\left(x ; x^{\prime}\right) & =\left\langle\Omega\left|\hat{A}_{0}(\eta, \vec{x}) \hat{A}_{0}\left(\eta^{\prime}, \vec{x}^{\prime}\right)\right| \Omega\right\rangle=\frac{\left(a a^{\prime}\right)^{2-D}}{(q \bar{\phi})^{2}\left(q \bar{\phi}^{\prime}\right)^{2}} \partial_{i} \partial_{j}^{\prime}\left\langle\Omega\left|\hat{\Pi}_{i}^{\mathrm{L}}(\eta, \vec{x}) \hat{\Pi}_{j}^{\mathrm{L}}\left(\eta^{\prime}, \vec{x}^{\prime}\right)\right| \Omega\right\rangle \\
& =-\frac{\left(a a^{\prime}\right)^{-\frac{(D-4) \epsilon}{2}}}{\left(q \bar{\phi}_{0}\right)^{2}} \nabla^{2} \mathcal{F}_{\nu}\left(y_{-+}\right), \\
i\left[{ }_{0}^{-} \Delta_{i}^{+}\right]\left(x ; x^{\prime}\right) & =\left\langle\Omega\left|\hat{A}_{0}(\eta, \vec{x}) \hat{A}_{i}\left(\eta^{\prime}, \vec{x}^{\prime}\right)\right| \Omega\right\rangle=\frac{\left(a a^{\prime}\right)^{2-D}}{(q \bar{\phi})^{2}\left(q \bar{\phi}^{\prime}\right)^{2}} \partial_{j} \partial_{0}^{\prime}\left\langle\Omega\left|\hat{\Pi}_{j}^{\mathrm{L}}(\eta, \vec{x}) \hat{\Pi}_{i}^{\mathrm{L}}\left(\eta^{\prime}, \vec{x}^{\prime}\right)\right| \Omega\right\rangle \\
& =-\frac{\left(a a^{\prime}\right)^{2-D+2 \epsilon}}{\left(q \bar{\phi}_{0}\right)^{2}} \partial_{i}^{\prime} \partial_{0}^{\prime}\left[\left(a a^{\prime}\right)^{D-2-\frac{D \epsilon}{2}} \mathcal{F}_{\nu}\left(y_{-+}\right)\right], \\
i\left[{ }_{i}^{-} \Delta_{j}^{+}\right]\left(x ; x^{\prime}\right) & =\left\langle\Omega\left|\hat{A}_{i}(\eta, \vec{x}) \hat{A}_{j}\left(\eta^{\prime}, \vec{x}^{\prime}\right)\right| \Omega\right\rangle \\
& =\left\langle\Omega\left|\hat{A}_{i}^{\mathrm{T}}(\eta, \vec{x}) \hat{A}_{j}^{\mathrm{T}}\left(\eta^{\prime}, \vec{x}^{\prime}\right)\right| \Omega\right\rangle+\frac{\left(a a^{\prime}\right)^{2-D}}{(q \bar{\phi})^{2}\left(q \bar{\phi}^{\prime}\right)^{2}} \partial_{0} \partial_{0}^{\prime}\left\langle\Omega\left|\hat{\Pi}_{i}^{\mathrm{L}}(\eta, \vec{x}) \hat{\Pi}_{j}^{\mathrm{L}}\left(\eta^{\prime}, \vec{x}^{\prime}\right)\right| \Omega\right\rangle
\end{aligned}
$$




$$
\begin{aligned}
=\left(a a^{\prime}\right)^{1-\frac{(D-2) \epsilon}{2}} \delta_{i j} \mathcal{F}_{\nu}\left(y_{-+}\right) & \\
+\frac{\left(a a^{\prime}\right)^{-\frac{(D-4) \epsilon}{2}}}{\left(q \bar{\phi}_{0}\right)^{2}} \frac{\partial_{i} \partial_{j}^{\prime}}{\nabla^{2}}\{[ & {\left[\frac{1}{4}(D-3-\epsilon)^{2}-(1-\epsilon)^{2} \nu^{2}\right] \mathcal{H} \mathcal{H}^{\prime} \mathcal{F}_{\nu}\left(y_{-+}\right) } \\
& \left.-\left(a a^{\prime}\right)^{2-D+\frac{D \epsilon}{2}} \partial_{0} \partial_{0}^{\prime}\left[\left(a a^{\prime}\right)^{D-2-\frac{D \epsilon}{2}} \mathcal{F}_{\nu}\left(y_{-+}\right)\right]\right\} .
\end{aligned}
$$

The component $i\left[{ }_{i}^{-} \Delta_{0}^{+}\right]\left(x ; x^{\prime}\right)$ is obtained from (7.35) by complex conjugation. Furthermore, using the identity (C.11) from appendix C for the special case $\lambda=\nu$ and $\rho=D-2-(D \epsilon) / 2$,

$$
\begin{aligned}
\partial_{0} \partial_{0}^{\prime}\left[\left(a a^{\prime}\right)^{D-2-\frac{D \epsilon}{2}} \mathcal{F}_{\nu}(y)\right]=\left(a a^{\prime}\right)^{D-2-\frac{D \epsilon}{2}} & \left\{\left[\frac{1}{4}(D-3-\epsilon)^{2}-(1-\epsilon)^{2} \nu^{2}\right] \mathcal{H H}^{\prime} \mathcal{F}_{\nu}\right. \\
& \left.-\frac{1}{2} \nabla^{2} I\left[(2-y) \frac{\partial \mathcal{F}_{\nu}}{\partial y}-\frac{1}{2}\left(D+\frac{D-4}{1-\epsilon}\right) \mathcal{F}_{\nu}\right]\right\},
\end{aligned}
$$

where $I[f(y)]=\int{ }^{y} d y^{\prime} f\left(y^{\prime}\right)$ denotes the primitive function, allows us to eliminate the inverse Laplacian in the $(i j)$ component (7.36), which now reads,

$$
\begin{aligned}
i\left[{ }_{i}^{-} \Delta_{j}^{+}\right]\left(x ; x^{\prime}\right)= & \left(a a^{\prime}\right)^{1-\frac{(D-2) \epsilon}{2}} \delta_{i j} \mathcal{F}_{\nu}\left(y_{-+}\right) \\
& +\frac{\left(a a^{\prime}\right)^{-\frac{(D-4) \epsilon}{2}}}{2\left(q \bar{\phi}_{0}\right)^{2}} \partial_{i} \partial_{j}^{\prime} I\left[\left(2-y_{-+}\right) \frac{\partial \mathcal{F}_{\nu}\left(y_{-+}\right)}{\partial y}-\left(2+\frac{(D-4)(2-\epsilon)}{2(1-\epsilon)}\right) \mathcal{F}_{\nu}\left(y_{-+}\right)\right] .
\end{aligned}
$$

\subsection{Covariantizing two-point functions}

Expressions (7.34), (7.35), and (7.38) represent the solutions for the covariant positivefrequency Wightman function of vector perturbations in the unitary gauge. For practical computations, however, it is advantageous to have it written in a manifestly covariant form, which we derive in this section. Subsequently, we derive the covariant form for the Feynman propagator, and for the $(++)$ Green's function. The covariant form is not expressible solely in terms of the distance function $y$ from (2.11) (as it would be in the de Sitter limit), but rather we need to introduce two other bi-local quantities, ${ }^{16}$

$$
u\left(x ; x^{\prime}\right)=(1-\epsilon) \ln \left(a a^{\prime}\right), \quad v\left(x ; x^{\prime}\right)=(1-\epsilon) \ln \left(a / a^{\prime}\right) .
$$

The appropriate tensor basis in which to express the Wightman function is,

$$
\begin{aligned}
i\left[{ }_{\mu} \Delta_{\nu}^{+}\right]\left(x ; x^{\prime}\right)= & \left(\partial_{\mu} \partial_{\nu}^{\prime} y_{-+}\right) \times \mathcal{C}_{1}\left(y_{-+}, u\right)+\left(\partial_{\mu} y_{-+}\right)\left(\partial_{\nu}^{\prime} y_{-+}\right) \times \mathcal{C}_{2}\left(y_{-+}, u\right) \\
& +\left[\left(\partial_{\mu} y_{-+}\right)\left(\partial_{\nu}^{\prime} u\right)+\left(\partial_{\mu} u\right)\left(\partial_{\nu}^{\prime} y_{-+}\right)\right] \times \mathcal{C}_{3}\left(y_{-+}, u\right)+\left(\partial_{\mu} u\right)\left(\partial_{\nu}^{\prime} u\right) \times \mathcal{C}_{4}\left(y_{-+}, u\right),
\end{aligned}
$$

where $\mathcal{C}_{i}$ 's are the scalar structure functions. Determining the four scalar structure functions is a matter of comparing the form above with expressions (7.34), (7.35), and (7.38)

${ }^{16}$ These quantities can be used to express the cosmological plasma 4-vectors,

$$
u_{\mu}(\eta)=\frac{\partial_{\mu} u\left(x ; x^{\prime}\right)}{(1-\epsilon) H(\eta)}=\frac{\partial_{\mu} v\left(x ; x^{\prime}\right)}{(1-\epsilon) H(\eta)}, \quad u_{\nu}\left(\eta^{\prime}\right)=\frac{\partial_{\nu}^{\prime} u\left(x ; x^{\prime}\right)}{(1-\epsilon) H\left(\eta^{\prime}\right)}=-\frac{\partial_{\nu}^{\prime} v\left(x ; x^{\prime}\right)}{(1-\epsilon) H\left(\eta^{\prime}\right)},
$$

which are canonically normalized, $g^{\mu \nu} u_{\mu} u_{\nu}=-1$, and the energy-momentum tensor of the classical cosmological fluid can be expressed in terms of them, $T_{\mu \nu}=(p+\rho) u_{\mu} u_{\nu}+p g_{\mu \nu}$. 
for the components of the Wightman function. In order to facilitate the comparison we first rewrite the components of the covariant expression (7.40) as,

$$
\begin{aligned}
i\left[{ }_{i}^{-} \Delta_{j}^{+}\right]\left(x ; x^{\prime}\right)= & -2 \delta_{i j}(1-\epsilon)^{2} H_{0}^{2} e^{u}\left\{\mathcal{C}_{1}\left(y_{-+}, u\right)-I\left[\mathcal{C}_{2}\right]\left(y_{-+}, u\right)\right\}+\partial_{i} \partial_{j}^{\prime} I^{2}\left[\mathcal{C}_{2}\right]\left(y_{-+}, u\right), \\
i\left[{ }_{0}^{-} \Delta_{i}^{+}\right]\left(x ; x^{\prime}\right)=(1-\epsilon) H_{0} e^{\frac{u+v}{2}}\left(\partial_{i}^{\prime} y_{-+}\right) & \times\left\{\mathcal{C}_{1}\left(y_{-+}, u\right)+\left[2 e^{-v}-(2-y)\right] \mathcal{C}_{2}\left(y_{-+}, u\right)+\mathcal{C}_{3}\left(y_{-+}, u\right)\right\} \\
i\left[{ }_{0}^{-} \Delta_{0}^{+}\right]\left(x ; x^{\prime}\right)=(1-\epsilon)^{2} H_{0}^{2} e^{u}\{[ & {\left[4 \operatorname{ch}(v)-\left(2-y_{-+}\right)\right] \mathcal{C}_{1}\left(y_{-+}, u\right) } \\
- & {\left[\left(4 y_{-+}-y_{-+}^{2}\right)+4 \operatorname{ch}(v)\left(2-y_{-+}\right)-8\right] \mathcal{C}_{2}\left(y_{-+}, u\right) } \\
+ & \left.2\left[2 \operatorname{ch}(v)-\left(2-y_{-+}\right)\right] \mathcal{C}_{3}\left(y_{-+}, u\right)+\mathcal{C}_{4}\left(y_{-+}, u\right)\right\}
\end{aligned}
$$

This form is obtained by using the chain rule, and the partial derivatives (C.2)-(C.8) from appendix C. We also rewrite (7.34), (7.35), and (7.38) in the analogous form in terms of $u$ and $v$ variables,

$$
\begin{aligned}
i\left[{ }_{i}^{-} \Delta_{j}^{+}\right]\left(x ; x^{\prime}\right)= & -2 \delta_{i j}(1-\epsilon)^{2} H_{0}^{2} e^{u} \times \frac{e^{-\frac{(D-4) \epsilon}{2(1-\epsilon)} u}}{2\left(q \bar{\phi}_{0}\right)^{2}}\left\{\left[\nu^{2}-\left(\frac{D-3-\epsilon}{2(1-\epsilon)}\right)^{2}\right] \mathcal{F}_{\nu}\left(y_{-+}\right)\right\} \\
& +\frac{e^{-\frac{(D-4) \epsilon}{2(1-\epsilon)} u}}{2\left(q \bar{\phi}_{0}\right)^{2}} \partial_{i} \partial_{j}^{\prime} I\left[\left(2-y_{-+}\right) \frac{\partial \mathcal{F}_{\nu}\left(y_{-+}\right)}{\partial y_{-+}}-\left(\frac{2(D-2)-D \epsilon}{2(1-\epsilon)}\right) \mathcal{F}_{\nu}\left(y_{-+}\right)\right] \\
i\left[{ }_{0}^{-} \Delta_{i}^{+}\right]\left(x ; x^{\prime}\right)= & (1-\epsilon) H_{0} e^{\frac{u+v}{2}}\left(\partial_{i}^{\prime} y_{-+}\right) \times \frac{e^{-\frac{(D-4) \epsilon}{2(1-\epsilon)} u}}{\left(q \bar{\phi}_{0}\right)^{2}}\left\{\left[\left(2-y_{-+}\right) e^{-v}-2\right] \frac{\partial^{2}}{\partial y_{-+}^{2}}\right. \\
i\left[{ }_{0} \Delta_{0}^{+}\right]\left(x ; x^{\prime}\right)= & \left.(1-\epsilon)^{2} H_{0}^{2} e^{u} \times \quad\left(\frac{2(D-1)-(D+2) \epsilon}{2(1-\epsilon)}\right) e^{-v} \frac{\partial}{\partial y_{-+}}\right\} \mathcal{F}_{\nu}\left(y_{-+}\right) \\
& \times \frac{e^{-\frac{(D-4) \epsilon}{2(1-\epsilon)} u}}{\left(q \bar{\phi}_{0}\right)^{2}}\left\{4\left[\left(2-y_{-+}\right)-2 \operatorname{ch}(v)\right] \frac{\partial^{2}}{\partial y_{-+}^{2}}-2(D-1) \frac{\partial}{\partial y_{-+}}\right\} \mathcal{F}_{\nu}\left(y_{-+}\right) .
\end{aligned}
$$

Now the first two structure functions $\mathcal{C}_{1}$ and $\mathcal{C}_{2}$ follow from comparing the expressions for the $(i j)$ component, $\mathcal{C}_{3}$ follows from comparing the $(0 i)$ component, and $\mathcal{C}_{4}$ from comparing the (00) component,

$$
\begin{aligned}
& \mathcal{C}_{1}(y, u)=\frac{e^{-\frac{(D-4) \epsilon}{2(1-\epsilon)} u}}{2\left(q \bar{\phi}_{0}\right)^{2}} \times {\left[-\left(4 y-y^{2}\right) \frac{\partial^{2}}{\partial y^{2}}-(D-1)(2-y) \frac{\partial}{\partial y}\right.} \\
&\left.-\frac{(D-4) \epsilon}{2(1-\epsilon)}\left(2+\frac{(D-4)(2-\epsilon)}{2(1-\epsilon)}\right)\right] \mathcal{F}_{\nu}(y), \\
& \mathcal{C}_{2}(y, u)=\frac{e^{-\frac{(D-4) \epsilon}{2(1-\epsilon)} u}}{2\left(q \bar{\phi}_{0}\right)^{2}} \times\left[(2-y) \frac{\partial^{2}}{\partial y^{2}}-\left(3+\frac{(D-4)(2-\epsilon)}{2(1-\epsilon)}\right) \frac{\partial}{\partial y}\right] \mathcal{F}_{\nu}(y),
\end{aligned}
$$




$$
\begin{aligned}
& \mathcal{C}_{3}(y, u)=\frac{e^{-\frac{(D-4) \epsilon}{2(1-\epsilon)} u}}{2\left(q \bar{\phi}_{0}\right)^{2}} \times \frac{(D-4) \epsilon}{2(1-\epsilon)}\left[-(2-y) \frac{\partial}{\partial y}+\left(2+\frac{(D-4)(2-\epsilon)}{2(1-\epsilon)}\right)\right] \mathcal{F}_{\nu}(y), \\
& \mathcal{C}_{4}(y, u)=\frac{e^{-\frac{(D-4) \epsilon}{2(1-\epsilon)} u}}{2\left(q \bar{\phi}_{0}\right)^{2}} \times \frac{(D-4) \epsilon}{2(1-\epsilon)}\left[\left(4 y-y^{2}\right) \frac{\partial}{\partial y}+\left(2+\frac{(D-4)(2-\epsilon)}{2(1-\epsilon)}\right)(2-y)\right] \mathcal{F}_{\nu}(y),
\end{aligned}
$$

where we have used eq. (B.4) in order to put $\mathcal{C}_{1}$ in the form (7.47). The above solutions for the four structure functions now specify the desired covariant positive-frequency Wightman function in (7.40) that we were after. Note that we could have assumed that the scalar structure functions in the covariant Ansatz (7.40) depends on variable $v$ as well, but we would have obtained the same solution above. The negative-frequency Wightman function is now obtained from the positive-frequency one by complex conjugation, which simply amounts to switching the $(-+)$ prescription in the distance function $y$ into the $(+-)$ one.

Any Wightman function has to satisfy the transversality relations (7.13), and the homogeneous equations of motion (7.16). Demonstrating that our solution for the Wightman function satisfies these conditions is a straightforward matter of applying identities (D.12)(D.13) to reduce the derivative order and the contraction identities from table 2 in appendix D. To demonstrate the equation of motion, in addition the differential equation (B.4) satisfied by the rescaled propagator function must be used.

The most straightforward way to obtain the Feynman propagator is to use its definition in terms of the positive- and negative-frequency Wightman functions,

$$
i\left[{ }_{\mu}^{+} \Delta_{\nu}^{+}\right]\left(x ; x^{\prime}\right)=\theta\left(\eta-\eta^{\prime}\right) i\left[{ }_{\mu}^{-} \Delta_{\nu}^{+}\right]\left(x ; x^{\prime}\right)+\theta\left(\eta^{\prime}-\eta\right) i\left[{ }_{\mu}^{+} \Delta_{\nu}^{-}\right]\left(x ; x^{\prime}\right) .
$$

We wish to write the Feynman propagator in the covariant form of $(7.40)$ with the $i \varepsilon$ prescription changed to the $(++)$ one from $(2.14)$. In order to arrive at that form, we need to commute the step functions in (7.51) through the derivatives in the tensor structures in (7.40), so that we can change the $i \varepsilon$-prescription in the basis tensors using,

$$
y_{++}=\theta\left(\eta-\eta^{\prime}\right) y_{-+}+\theta\left(\eta^{\prime}-\eta\right) y_{+-} .
$$

For the last three terms from (7.40) this exercise simply amounts to substituting all the $(-+)$ prescriptions with the $(++)$ ones. For the first term from $(7.40)$ this is more subtle, as the result of the commuting gives,

$$
\begin{aligned}
& \theta\left(\eta-\eta^{\prime}\right)\left(\partial_{\mu} \partial_{\nu}^{\prime} y_{-+}\right) \mathcal{C}_{1}\left(y_{-+}, u\right)+\theta\left(\eta^{\prime}-\eta\right)\left(\partial_{\mu} \partial_{\nu}^{\prime} y_{+-}\right) \mathcal{C}_{1}\left(y_{+-}, u\right) \\
& =\left[\left(\partial_{\mu} \partial_{\nu}^{\prime} y_{++}\right)+\delta_{\mu}^{0} \delta_{\nu}^{0}(1-\epsilon)^{2} \mathcal{H}^{2} \times i \varepsilon \delta\left(\eta-\eta^{\prime}\right)\right] \mathcal{C}_{1}\left(y_{++}, u\right) \equiv \overline{\left(\partial_{\mu} \partial_{\nu}^{\prime} y_{-+}\right)} \mathcal{C}_{1}\left(y_{++}, u\right)
\end{aligned}
$$

The extra term that comes together with the tensor structure above seems irrelevant at a first glance, on the account of containing the infinitesimal $i \varepsilon$. However, given that the most singular term in the first scalar structure function (7.47) is $\propto y_{++}^{D / 2}$ we absolutely must keep the extra term, as it harbors a delta-function according to the identity,

$$
i \varepsilon \delta\left(\eta-\eta^{\prime}\right) \times\left(\frac{y_{++}}{4}\right)^{-\frac{D}{2}}=[(1-\epsilon) H]^{-D} \frac{(4 \pi)^{D / 2}}{\Gamma\left(\frac{D}{2}\right)} \times \frac{i \delta^{D}\left(x-x^{\prime}\right)}{\sqrt{-g}} .
$$


Therefore, the Feynman propagator is obtained from the Wightman function (7.40) by switching all the Schwinger-Keldysh polarity labels from $(-+)$ to $(++)$, and by using the modified first tensor structure from (7.53),

$$
\begin{aligned}
i\left[{ }_{\mu}^{+} \Delta_{\nu}^{+}\right]\left(x ; x^{\prime}\right)= & \overline{\left(\partial_{\mu} \partial_{\nu}^{\prime} y_{++}\right)} \times \mathcal{C}_{1}\left(y_{++}, u\right)+\left(\partial_{\mu} y_{++}\right)\left(\partial_{\nu}^{\prime} y_{++}\right) \times \mathcal{C}_{2}\left(y_{++}, u\right) \\
& +\left[\left(\partial_{\mu} y_{++}\right)\left(\partial_{\nu}^{\prime} u\right)+\left(\partial_{\mu} u\right)\left(\partial_{\nu}^{\prime} y_{++}\right)\right] \times \mathcal{C}_{3}\left(y_{++}, u\right)+\left(\partial_{\mu} u\right)\left(\partial_{\nu}^{\prime} u\right) \times \mathcal{C}_{4}\left(y_{++}, u\right) .
\end{aligned}
$$

The $(++)$ Green's function is now constructed from the above Feynman one, and with the use of the identity (7.54) for the delta-function,

$$
\begin{aligned}
i\left[{ }_{\mu}^{+} G_{\nu}^{+}\right]\left(x ; x^{\prime}\right)= & \left(\partial_{\mu} \partial_{\nu}^{\prime} y_{++}\right) \times \mathcal{C}_{1}\left(y_{++}, u\right)+\left(\partial_{\mu} y_{++}\right)\left(\partial_{\nu}^{\prime} y_{++}\right) \times \mathcal{C}_{2}\left(y_{++}, u\right) \\
& +\left[\left(\partial_{\mu} y_{++}\right)\left(\partial_{\nu}^{\prime} u\right)+\left(\partial_{\mu} u\right)\left(\partial_{\nu}^{\prime} y_{++}\right)\right] \times \mathcal{C}_{3}\left(y_{++}, u\right)+\left(\partial_{\mu} u\right)\left(\partial_{\nu}^{\prime} u\right) \times \mathcal{C}_{4}\left(y_{++}, u\right)
\end{aligned}
$$

Note that it is the $(++)$ Green's function that we obtain from the Wightman function by the naîve switch of the polarity labels, and not the Feynman propagator.

Checking that the Feynman propagator satisfies the correct transversality relations (7.14)-(7.15), and the correct equations of motion (7.17)-(7.18) is a tedious task of pedantically applying identities provided in appendix D. To confirm the transversality relations we first use identities (D.14)-(D.18) to isolate the delta functions in time descending from time derivatives acting on the $(++) i \varepsilon$-prescription, and to reduce the derivative order in the tensor structures. Then we use the identities from table 2 to perform the necessary tensor contractions. Lastly, we use the solutions for the scalar structure functions, together with the identities (D.6)-(D.11) to recognize the space-time delta functions. To confirm the equations of motion satisfied by the Feynman propagator, we follow the same sequence of identities, with the additions of using the differential equation for the rescaled propagator function (B.4) in the end. Had we not been careful about the definition of the tensor structures and the $i \varepsilon$-prescriptions utilized in them, we would not have reproduced the correct transversality relations and equations of motion dictated by the canonical quantization.

\subsection{Various limits}

Anticipating future computations, this section provides various limits of the Wightman function (7.40) and the Feynman propagator (7.55), with scalar structure functions given in (7.47)-(7.50), namely the de Sitter limit, the flat space limit, and the coincidence limit. Computing the latter two involves examining the two-point function around $y \sim 0$, hence it is very convenient to have a power series representation of the rescaled propagator function $\mathcal{F}_{\nu}(y)$ that is derived in appendix B,

$$
\begin{aligned}
\mathcal{F}_{\nu}(y)= & \frac{\left[(1-\epsilon) H_{0}\right]^{D-2}}{(4 \pi)^{D / 2}} \Gamma\left(\frac{D-2}{2}\right) \times\left\{\left(\frac{y}{4}\right)^{-\frac{D-2}{2}}+\frac{\Gamma\left(\frac{4-D}{2}\right)}{\Gamma\left(\frac{1}{2}+\nu\right) \Gamma\left(\frac{1}{2}-\nu\right)} \times\right. \\
& \left.\times \sum_{n=0}^{\infty}\left[\frac{\Gamma\left(\frac{3}{2}+\nu+n\right) \Gamma\left(\frac{3}{2}-\nu+n\right)}{\Gamma\left(\frac{6-D}{2}+n\right)(n+1) !}\left(\frac{y}{4}\right)^{n-\frac{D-4}{2}}-\frac{\Gamma\left(\frac{D-1}{2}+\nu+n\right) \Gamma\left(\frac{D-1}{2}-\nu+n\right)}{\Gamma\left(\frac{D}{2}+n\right) n !}\left(\frac{y}{4}\right)^{n}\right]\right\} .
\end{aligned}
$$


The limit $D \rightarrow 4$ of the two-point functions (7.40) and (7.55) is trivial, and only the first two structure functions contribute to it. The limit of vanishing condensate (massless limit) is not accessible due to the unitary gauge being singular in this limit.

\subsubsection{De Sitter limit}

The de Sitter limit of the two-point functions (7.40), (7.55), and (7.56) simply amounts to setting $\epsilon=0$ everywhere, including the distance function (2.13). The last two scalar structure functions vanish in this limit, and the two-point functions take a manifestly de Sitter-invariant form,

$$
\begin{aligned}
& i\left[{ }_{\mu}^{-} \Delta_{\nu}^{+}\right]\left(x ; x^{\prime}\right) \stackrel{\epsilon \rightarrow 0}{\longrightarrow}\left(\partial_{\mu} \partial_{\nu}^{\prime} y_{-+}\right) \times \mathcal{C}_{1}^{\mathrm{dS}}\left(y_{-+}\right)+\left(\partial_{\mu} y_{-+}\right)\left(\partial_{\nu}^{\prime} y_{-+}\right) \times \mathcal{C}_{2}^{\mathrm{dS}}\left(y_{-+}\right), \\
& i\left[{ }_{\mu}^{+} \Delta_{\nu}^{+}\right]\left(x ; x^{\prime}\right) \stackrel{\epsilon \rightarrow 0}{\longrightarrow} \overline{\left(\partial_{\mu} \partial_{\nu}^{\prime} y_{++}\right)} \times \mathcal{C}_{1}^{\mathrm{dS}}\left(y_{++}\right)+\left(\partial_{\mu} y_{++}\right)\left(\partial_{\nu}^{\prime} y_{++}\right) \times \mathcal{C}_{2}^{\mathrm{dS}}\left(y_{++}\right) \text {, } \\
& i\left[{ }_{\mu}^{+} G_{\nu}^{+}\right]\left(x ; x^{\prime}\right) \stackrel{\epsilon \rightarrow 0}{\longrightarrow}\left(\partial_{\mu} \partial_{\nu}^{\prime} y_{++}\right) \times \mathcal{C}_{1}^{\mathrm{dS}}\left(y_{++}\right)+\left(\partial_{\mu} y_{++}\right)\left(\partial_{\nu}^{\prime} y_{++}\right) \times \mathcal{C}_{2}^{\mathrm{dS}}\left(y_{++}\right),
\end{aligned}
$$

where the two structure functions are,

$$
\begin{aligned}
& \mathcal{C}_{1}^{\mathrm{dS}}(y)=\lim _{\epsilon \rightarrow 0} \mathcal{C}_{1}(y, u)=\frac{1}{2\left(q \bar{\phi}_{0}\right)^{2}}\left[-\left(4 y-y^{2}\right) \frac{\partial^{2}}{\partial y^{2}}-(D-1)(2-y) \frac{\partial}{\partial y}\right] \mathcal{F}_{\nu_{0}}^{\mathrm{dS}}(y), \\
& \mathcal{C}_{2}^{\mathrm{dS}}(y)=\lim _{\epsilon \rightarrow 0} \mathcal{C}_{2}(y, u)=\frac{1}{2\left(q \bar{\phi}_{0}\right)^{2}}\left[(2-y) \frac{\partial^{2}}{\partial y^{2}}-(D-1) \frac{\partial}{\partial y}\right] \mathcal{F}_{\nu_{0}}^{\mathrm{dS}}(y),
\end{aligned}
$$

where $\mathcal{F}_{\nu_{0}}^{\mathrm{dS}}(y)$ is obtained from $\mathcal{F}_{\lambda}(y)$ defined in eq. (5.5) by setting $\epsilon=0$ and $\lambda \rightarrow \nu_{0}$, and where the index (6.36) simplifies to,

$$
\nu_{0}=\sqrt{\left(\frac{D-3}{2}\right)^{2}-\left(\frac{q \bar{\phi}_{0}}{H_{0}}\right)^{2}}, \quad\left(\frac{q \bar{\phi}_{0}}{H_{0}}\right)^{2}=-D(D-1) \frac{q^{2} \xi}{\lambda} .
$$

This implies that in de Sitter we need $\xi<0$, such that the scalar potential has a Mexican hat form, which harbors a symmetry-breaking minimum. The transversality relations (7.13)-(7.15) and (7.20), and the equations of motion (7.16)-(7.18) and (7.21) have a straightforward de Sitter limit as well.

\subsubsection{Flat space limit}

The naîve flat space limit corresponds to taking $H_{0} \rightarrow 0$. However, that would correspond to the singular point of the unitary gauge in flat space, as in that limit the scalar condensate (4.4) also vanishes. In order to obtain a meaningful expression we need to consider the formal flat space limit where the non-minimal coupling reduces to the tachyonic mass term, $\xi R \stackrel{H_{0} \rightarrow 0}{\longrightarrow} \mathcal{M}^{2}<0$, which thus produces the usual symmetry-breaking potential. Therefore, the flat space limit for the photon two-point function needs to be taken as,

$$
\left(q \bar{\phi}_{0}\right)^{2} \stackrel{H_{0} \rightarrow 0}{\longrightarrow} m^{2}>0 .
$$

In the flat space limit we have for the variables (2.11) and (7.39),

$$
\begin{aligned}
& y\left(x ; x^{\prime}\right) \stackrel{H_{0} \rightarrow 0}{\sim}(1-\epsilon)^{2} H_{0}^{2}\left(\Delta x^{2}\right), \\
& u\left(x ; x^{\prime}\right) \stackrel{H_{0} \rightarrow 0}{\sim}(1-\epsilon) H_{0}\left(\eta+\eta^{\prime}-2 \eta_{0}\right), \\
& v\left(x ; x^{\prime}\right) \stackrel{H_{0} \rightarrow 0}{\sim}(1-\epsilon) H_{0}\left(\eta-\eta^{\prime}\right),
\end{aligned}
$$


for the tensor basis,

$$
\begin{aligned}
\overline{\left(\partial_{\mu} \partial_{\nu}^{\prime} y_{++}\right)},\left(\partial_{\mu} \partial_{\nu}^{\prime} y_{-+}\right) & \stackrel{H_{0} \rightarrow 0}{\sim}-2\left[(1-\epsilon) H_{0}\right]^{2} \eta_{\mu \nu}, \\
\left(\partial_{\mu} y_{A B}\right)\left(\partial_{\nu}^{\prime} y_{A B}\right) & \stackrel{H_{0} \rightarrow 0}{\sim}\left[(1-\epsilon) H_{0}\right]^{4}\left(\partial_{\mu} \Delta x_{A B}^{2}\right)\left(\partial_{\nu}^{\prime} \Delta x_{A B}^{\sigma}\right), \\
{\left[\left(\partial_{\mu} y_{A B}\right)\left(\partial_{\nu}^{\prime} u\right)+\left(\partial_{\mu} u\right)\left(\partial_{\nu}^{\prime} y_{A B}\right)\right] } & \stackrel{H_{0} \rightarrow 0}{\sim}\left[(1-\epsilon) H_{0}\right]^{3}\left[\left(\partial_{\mu} \Delta x_{A B}^{2}\right) \delta_{\nu}^{0}+\delta_{\mu}^{0}\left(\partial_{\nu}^{\prime} y\right)\right], \\
\left(\partial_{\mu} u\right)\left(\partial_{\nu}^{\prime} u\right) & \stackrel{H_{0} \rightarrow 0}{\sim}\left[(1-\epsilon) H_{0}\right]^{2} \delta_{\mu}^{0} \delta_{\nu}^{0} .
\end{aligned}
$$

and for the partial derivatives in the scalar structure functions,

$$
\frac{\partial}{\partial y}=\frac{1}{(1-\epsilon)^{2} \mathcal{H} \mathcal{H}^{\prime}} \frac{\partial}{\partial\left(\Delta x^{2}\right)} \stackrel{H_{0} \rightarrow 0}{\sim} \frac{1}{(1-\epsilon)^{2} H_{0}^{2}} \frac{\partial}{\partial\left(\Delta x^{2}\right)},
$$

The flat space limit of the rescaled propagator function is obtained from the power series representation (7.57), where the dependence on $H_{0}$ is found in powers of $y$ and within the gamma functions inside the index $\nu$,

$$
\nu=\sqrt{\left(\frac{D-3-\epsilon}{2(1-\epsilon)}\right)^{2}-\frac{m^{2}}{(1-\epsilon)^{2} H_{0}^{2}}} \stackrel{H_{0} \rightarrow 0}{\sim} \frac{i m}{(1-\epsilon) H_{0}} .
$$

The key is to examine the ratio of the gamma functions that appears in the power series, and make use of 8.328.1 from [104] to take the limit,

$$
\frac{\Gamma(\alpha+\nu+n) \Gamma(\alpha-\nu+n)}{\Gamma\left(\frac{1}{2}+\nu\right) \Gamma\left(\frac{1}{2}-\nu\right)} \stackrel{H_{0} \rightarrow 0}{\sim}\left[\frac{m}{(1-\epsilon) H_{0}}\right]^{2 n+2 \alpha-1},
$$

after which the series (7.57) reduces to

$$
\begin{aligned}
\mathcal{F}_{\nu}(y) \stackrel{H_{0} \rightarrow 0}{\longrightarrow} & \frac{\Gamma\left(\frac{D-2}{2}\right) \Gamma\left(\frac{4-D}{2}\right)}{(4 \pi)^{D / 2}} \\
& \times \sum_{n=0}^{\infty}\left[\frac{m^{2 n}}{\Gamma\left(\frac{4-D}{2}+n\right) n !}\left(\frac{\Delta x^{2}}{4}\right)^{n-\frac{D-2}{2}}-\frac{m^{2 n+D-2}}{\Gamma\left(\frac{D}{2}+n\right) n !}\left(\frac{\Delta x^{2}}{4}\right)^{n}\right] .
\end{aligned}
$$

This is recognized as a power series representation of the modified Bessel function of the second kind, according to 8.445 and 8.407.1 from [104],

$$
\mathcal{F}_{\nu}(y) \stackrel{H_{0} \rightarrow 0}{\longrightarrow} \frac{1}{(2 \pi)^{D / 2}}\left(\frac{m^{2}}{\Delta x^{2}}\right)^{\frac{D-2}{4}} K_{\frac{D-2}{2}}\left(\sqrt{m^{2} \Delta x^{2}}\right) \equiv i \Delta_{m}\left(\Delta x^{2}\right),
$$

which corresponds to the propagator function for the vacuum state of the massive scalar field in flat space (after inserting the appropriate $i \varepsilon$-prescription),

$$
\left(\square-m^{2}\right) i \Delta_{m}\left(\Delta x_{-+}^{2}\right)=0, \quad\left(\square-m^{2}\right) i \Delta_{m}\left(\Delta x_{++}^{2}\right)=i \delta^{D}\left(x-x^{\prime}\right),
$$

where in flat space $\square=\eta^{\mu \nu} \partial_{\mu} \partial_{\nu}$. 
Only the first two structure functions contribute in this limit,

$$
\begin{aligned}
& \mathcal{C}_{1}(u, y) \stackrel{H_{0} \rightarrow 0}{\sim} \frac{1}{\left[(1-\epsilon) H_{0}\right]^{2}} \times \frac{1}{m^{2}}\left[-2 \Delta x^{2} \frac{\partial^{2}}{\partial\left(\Delta x^{2}\right)^{2}}-(D-1) \frac{\partial}{\partial\left(\Delta x^{2}\right)}\right] i \Delta_{m}\left(\Delta x^{2}\right), \\
& \mathcal{C}_{2}(u, y) \stackrel{H_{0} \rightarrow 0}{\sim} \frac{1}{\left[(1-\epsilon) H_{0}\right]^{4}} \times \frac{1}{m^{2}}\left[\frac{\partial^{2}}{\partial\left(\Delta x^{2}\right)^{2}}\right] i \Delta_{m}\left(\Delta x^{2}\right) .
\end{aligned}
$$

Therefore, the flat space limit of the vector two-point functions is

$$
\begin{aligned}
i\left[{ }_{\mu}^{A} \Delta_{\nu}^{B}\right]\left(x ; x^{\prime}\right) \stackrel{H_{0} \rightarrow 0}{\longrightarrow} \frac{2}{m^{2}}\{ & \eta_{\mu \nu}\left[2\left(\Delta x_{A B}^{2}\right) \frac{\partial^{2}}{\partial\left(\Delta x_{A B}^{2}\right)^{2}}+(D-1) \frac{\partial}{\partial\left(\Delta x_{A B}^{2}\right)}\right] \\
& \left.-2\left(\Delta x_{A B}\right)_{\mu}\left(\Delta x_{A B}\right)_{\nu} \frac{\partial^{2}}{\partial\left(\Delta x_{A B}^{2}\right)^{2}}\right\} i \Delta_{m}\left(\Delta x_{A B}^{2}\right) .
\end{aligned}
$$

The flat space limit of the Wightman function can be written in a more standard form making use of (7.77),

$$
i\left[{ }_{\mu}^{-} \Delta_{\nu}^{+}\right]\left(x ; x^{\prime}\right) \stackrel{H_{0} \rightarrow 0}{\longrightarrow}\left(\eta_{\mu \nu}-\frac{\partial_{\mu} \partial_{\nu}}{m^{2}}\right) i \Delta_{m}\left(\Delta x_{-+}^{2}\right) .
$$

The same can be done for the Feynman propagator, carefully accounting for (7.77),

$$
i\left[{ }_{\mu}^{+} \Delta_{\nu}^{+}\right]\left(x ; x^{\prime}\right) \stackrel{H_{0} \rightarrow 0}{\longrightarrow}\left(\eta_{\mu \nu}-\frac{\partial_{\mu} \partial_{\nu}}{m^{2}}\right) i \Delta_{m}\left(\Delta x_{++}^{2}\right)-\frac{\delta_{\mu}^{0} \delta_{\nu}^{0}}{m^{2}} i \delta^{D}\left(x-x^{\prime}\right),
$$

which indeed is the form the propagator is supposed to take (see e.g. section 6.5 from [70]). This represents the second non-trivial check of our principal result (7.40) and (7.47)-(7.50). The $(++)$ Green's function in flat space (7.56) now takes the form,

$$
i\left[{ }_{\mu}^{+} G_{\nu}^{+}\right]\left(x ; x^{\prime}\right) \stackrel{H_{0} \rightarrow 0}{\longrightarrow}\left(\eta_{\mu \nu}-\frac{\partial_{\mu} \partial_{\nu}}{m^{2}}\right) i \Delta_{m}\left(\Delta x_{++}^{2}\right) .
$$

\subsubsection{Coincidence limit}

The utilization of the Wightman function (7.40) and the Feynman propagator (7.55) in loop computations will require their dimensionally regulated coincidence limits, which we work out here. The coincident limit of the variables (2.11) and (7.39) is,

$$
y_{A B} \stackrel{x^{\prime} \rightarrow x}{\longrightarrow} 0, \quad u \stackrel{x^{\prime} \rightarrow x}{\longrightarrow} 2(1-\epsilon) \ln (a), \quad v \stackrel{x^{\prime} \rightarrow x}{\longrightarrow} 0,
$$

and of the basis tensors,

$$
\begin{aligned}
& \overline{\left(\partial_{\mu} \partial_{\nu}^{\prime} y_{-+}\right)},\left(\partial_{\mu} \partial_{\nu}^{\prime} y_{-+}\right) \stackrel{x^{\prime} \rightarrow x}{\longrightarrow}-2[(1-\epsilon) H]^{2} a^{2} \eta_{\mu \nu}, \quad\left(\partial_{\mu} y_{A B}\right)\left(\partial_{\nu}^{\prime} y_{A B}\right) \stackrel{x^{\prime} \rightarrow x}{\longrightarrow} 0, \\
& {\left[\left(\partial_{\mu} y_{A B}\right)\left(\partial_{\nu}^{\prime} u\right)+\left(\partial_{\mu} u\right)\left(\partial_{\nu}^{\prime} y_{A B}\right)\right] \stackrel{x^{\prime} \rightarrow x}{\longrightarrow} 0, \quad\left(\partial_{\mu} u\right)\left(\partial_{\nu}^{\prime} u\right) \stackrel{x^{\prime} \rightarrow x}{\longrightarrow}[(1-\epsilon) H]^{2} a^{2} \delta_{\mu}^{0} \delta_{\nu}^{0} .}
\end{aligned}
$$

It follows that only the structure functions $\mathcal{C}_{1}$ and $\mathcal{C}_{4}$ contribute to the coincident limit,

$$
\begin{aligned}
i\left[{ }_{\mu}^{A} \Delta_{\nu}^{B}\right](x ; x)= & g_{\mu \nu} \times(-2)[(1-\epsilon) H]^{2} \mathcal{C}_{1}(0,2 \ln (a)) \\
& +\left(a^{2} \delta_{\mu}^{0} \delta_{\nu}^{0}\right) \times[(1-\epsilon) H]^{2} \mathcal{C}_{4}(0,2 \ln (a)),
\end{aligned}
$$


where their dimensionally regulated coincident limits are,

$$
\begin{aligned}
(-2)[(1-\epsilon) H]^{2} \mathcal{C}_{1}(0,2 \ln (a))= & \frac{[(1-\epsilon) H]^{D-2}}{(4 \pi)^{D / 2}} \times \frac{\Gamma\left(\frac{D-1}{2}+\nu\right) \Gamma\left(\frac{D-1}{2}-\nu\right)}{\Gamma\left(\frac{1}{2}+\nu\right) \Gamma\left(\frac{1}{2}-\nu\right)} \times \Gamma\left(\frac{2-D}{2}\right) \\
& \times \frac{1}{D}\left[(D-1)+\left(D-1+\frac{(D-4) \epsilon}{2(1-\epsilon)}\right)\right. \\
& \left.\quad \times\left(2+\frac{(D-4)(2-\epsilon)}{2(1-\epsilon)}\right)\left(\frac{(1-\epsilon) H_{0}}{q \bar{\phi}_{0}}\right)^{2}\right], \quad(7.87) \\
{[(1-\epsilon) H]^{2} \mathcal{C}_{4}(0,2 \ln (a))=} & \frac{[(1-\epsilon) H]^{D-2}}{(4 \pi)^{D / 2}} \times \frac{\Gamma\left(\frac{D-1}{2}+\nu\right) \Gamma\left(\frac{D-1}{2}-\nu\right)}{\Gamma\left(\frac{1}{2}+\nu\right) \Gamma\left(\frac{1}{2}-\nu\right)} \times \Gamma\left(\frac{2-D}{2}\right) \\
& \times \frac{(D-4) \epsilon}{2(1-\epsilon)}\left(2+\frac{(D-4)(2-\epsilon)}{2(1-\epsilon)}\right)\left(\frac{(1-\epsilon) H_{0}}{q \bar{\phi}_{0}}\right)^{2} .
\end{aligned}
$$

These are obtained by (i) acting with derivative operators on in (7.47) and (7.50) on the power series representation for $\mathcal{F}_{\nu}(y)$ in (7.57), (ii) setting all the $D$-dependent powers of $y$ to zero, and (iii) setting all the positive powers of $y$ to zero. The coincident structure function $\mathcal{C}_{1}$ in (7.87) diverges as $\propto 1 /(D-4)$, which can be seen from the factor,

$$
\Gamma\left(\frac{2-D}{2}\right)=\frac{2}{D-4}-1+\gamma_{\mathrm{E}}+\mathcal{O}(D-4)
$$

where $\gamma_{\mathrm{E}} \simeq 0.577$ is the Euler-Mascheroni constant. The other coincident structure function $\mathcal{C}_{4}$ in $(7.88)$ is finite in this limit.

\subsection{Comparison with previous results}

The existing literature on massive vector field propagators in inflation pertains only to the de Sitter space. There are two works $[76,79]$ that we can directly compare to the de Sitter limit of our propagator in section 7.5.1. The first one is the classic work of Allen and Jacobson [76] where, among multiple results, the two-point function for the massive vector field in de Sitter is reported in their section III. This vector field is described by the action,

$$
S\left[A_{\mu}\right]=\int d^{D} x \sqrt{-g}\left[-\frac{1}{4} g^{\mu \nu} g^{\rho \sigma} F_{\mu \rho} F_{\nu \sigma}-\frac{m^{2}}{2} g^{\mu \nu} A_{\mu} A_{\nu}\right] .
$$

which is assumed to result from spontaneous symmetry breaking mechanism, and corresponds to the unitary gauge, as detailed here in section 3. Comparing this action to the covariant gauge-fixed action (3.45) expanded in perturbations, we see that the de Sitter limit of our two-point functions given in section 7.5.1 should reproduce the result from [76] upon the identification $m^{2} \rightarrow\left(q \bar{\phi}_{0}\right)^{2}=$ const. Allen and Jacobson construct the vector field two-point function by solving the equation of motion it satisfies off-coincidence at spacelike separations, under the warranted assumption of $D$-dimensional de Sitter isometries. No $i \varepsilon$-prescriptions are necessary in that case. In fact all three two-point functions (7.58)(7.60) we gave in section 7.5.1 coincide for space-like separations, and the only thing we need to check is that the scalar structure functions (7.61)-(7.62) are consistent with the 
result from [76]. When transcribed to the bi-tensor basis used here ${ }^{17}$ the two-point function from [76] reads,

$$
i\left[{ }_{\mu} \Delta_{\nu}\right]^{\mathrm{AJ}}\left(x ; x^{\prime}\right)=\left(\partial_{\mu} \partial_{\nu}^{\prime} y\right) \times \mathcal{C}_{1}^{\mathrm{AJ}}(y)+\left(\partial_{\mu} y\right)\left(\partial_{\nu}^{\prime} y\right) \times \mathcal{C}_{1}^{\mathrm{AJ}}(y),
$$

where the two scalar structure functions,

$$
\begin{aligned}
& \mathcal{C}_{1}^{\mathrm{AJ}}(y)=\frac{1}{4(D-1) H_{0}^{2}}\left[-\left(4 y-y^{2}\right) \frac{\partial}{\partial y}-(D-1)(2-y)\right] \gamma_{\mathrm{AJ}}(y), \\
& \mathcal{C}_{2}^{\mathrm{AJ}}(y)=\frac{1}{4(D-1) H_{0}^{2}}\left[(2-y) \frac{\partial}{\partial y}-(D-1)\right] \gamma_{\mathrm{AJ}}(y)
\end{aligned}
$$

are expressed in terms of a single scalar function,

$$
\begin{aligned}
\gamma_{\mathrm{AJ}}(y)= & \frac{(1-D) \Gamma\left(\frac{D+1}{2}+\nu_{\mathrm{AJ}}\right) \Gamma\left(\frac{D+1}{2}-\nu_{\mathrm{AJ}}\right)}{2^{D+1} \pi^{D / 2} \Gamma\left(\frac{D+2}{2}\right)} \\
& \times \frac{H_{0}^{D}}{m^{2}}{ }_{2} F_{1}\left(\left\{\frac{D+1}{2}+\nu_{\mathrm{AJ}}, \frac{D+1}{2}-\nu_{\mathrm{AJ}}\right\},\left\{\frac{D+2}{2}\right\}, 1-\frac{y}{4}\right),
\end{aligned}
$$

and

$$
\nu_{\mathrm{AJ}}=\sqrt{\left(\frac{D-3}{2}\right)^{2}-\frac{m^{2}}{H_{0}^{2}}} .
$$

Using the fact that derivatives of the hypergeometric function raises its parameters by one, we can recognize that function in (7.94) is

$$
\gamma_{\mathrm{AJ}}(y)=2(D-1) \frac{H_{0}^{2}}{m^{2}} \frac{\partial}{\partial y} \mathcal{F}_{\nu_{\mathrm{AJ}}}(y) .
$$

Now, upon identifying

$$
\frac{m^{2}}{H_{0}^{2}} \rightarrow\left(\frac{q \bar{\phi}_{0}}{H_{0}}\right)^{2}=-D(D-1) \frac{q^{2} \xi}{\lambda}
$$

the structure functions (7.92) and (7.93) match the de Sitter limit of our structure functions in (7.61) and (7.62), as they should.

It is necessary to restore the $i \varepsilon$-prescription for time-like separations and on the light cone, to distinguish between different two-point functions. In [76] the Feynman propagator is defined by setting $y \rightarrow y+i \varepsilon$ (the sign error in the original work was corrected in ref. [79]) in the scalar structure functions. The $i \varepsilon$-prescription in the tensor structures is, however, not discussed, and neither are the sourced equations of motion satisfied by the Feynman propagator. As pointed out in section 7.1 here, this is an important detail, which distinguishes between the Feynman propagator and the Green's function. This distinction is important in loop computations regarding renormalization, and the careful account of the $i \varepsilon$-prescription

\footnotetext{
${ }^{17}$ The relations between the basis tensors that are used in [76] and the ones we use here are given in ref. [77], and they read,

$$
z=1-\frac{y}{4}, \quad g_{\mu \nu^{\prime}}=-\frac{1}{2 H^{2}}\left(\partial_{\mu} \partial_{\nu}^{\prime} y\right)-\frac{1}{2 H^{2}(4-y)}\left(\partial_{\mu} y\right)\left(\partial_{\nu}^{\prime} y\right), \quad n_{\mu} n_{\nu^{\prime}}=\frac{1}{H^{2}\left(4 y-y^{2}\right)}\left(\partial_{\mu} y\right)\left(\partial_{\nu}^{\prime} y\right)
$$
}


in the tensor structures from this section supplements the classic result by Allen and Jacobson in this regard. The Allen-Jacobson massive vector propagator in de Sitter has also been reproduced using canonical methods in [102], and using Euclidean methods in [105].

The second work that we can compare to is due to Fröb and Higuchi [79] who report a vector field propagator for the Stueckelberg model in de Sitter in the adapted version of the covariant $R_{\xi}$ gauge (defined in (7.101) below). They report to reproduce the result by Allen and Jacobson in the unitary gauge limit $\alpha \rightarrow \infty$, so there is no need to explicitly check for that again. However, we comment on how the Stueckelberg model relates to the Abelian Higgs model in the de Sitter limit. The Stueckelberg model in curved space-time in given by the action

$$
S_{\mathrm{St} .}\left[A_{\mu}, \varphi\right]=\int d^{D} x \sqrt{-g}\left[-\frac{1}{4} g^{\mu \rho} g^{\nu \sigma} F_{\mu \nu} F_{\rho \sigma}-\frac{1}{2} g^{\mu \nu}\left(\partial_{\mu} \varphi-m A_{\mu}\right)\left(\partial_{\nu} \varphi-m A_{\nu}\right)\right],
$$

where $m$ is the mass parameter. It is invariant under gauge transformations,

$$
A_{\mu} \rightarrow A_{\mu}+\partial_{\mu} \Lambda, \quad \varphi \rightarrow \varphi+m \Lambda,
$$

where $\Lambda$ is an arbitrary function. This model in de Sitter space is equivalent to the linear level of our Abelian Higgs model. The connection is established by fixing the de Sitter background in the action (3.1) for the Abelian Higgs model, writing the complex scalar in the polar basis,

$$
\Phi=\frac{1}{\sqrt{2}}(\bar{\phi}+\psi) e^{-i \varphi / \bar{\phi}}
$$

where $\bar{\phi}=$ const. is the condensate, and expanding the action to quadratic order in the fluctuations $\psi$ and $\varphi$. The action for the modulus fluctuation $\psi$ decouples, and takes the form of (5.2) with $\epsilon=0$, while the action for the phase of the scalar, and the vector field take the form of the Stueckelberg action (7.98), provided we identify $m=q \bar{\phi}$.

The Stueckelberg model is a gauge theory, and requires gauge-fixing. The most convenient way of imposing the gauge in de Sitter seems to be a version of the $R_{\xi}$ gauge [54, 55], whereby the action (7.98) is substituted by the gauge-fixed action $S_{*}=S_{\mathrm{St} .}+S_{\mathrm{gf}}$, wit the gauge-fixing term,

$$
S_{\mathrm{gf}}\left[A_{\mu}, \varphi\right]=\int d^{D} x\left[-\frac{1}{2 \alpha}\left(g^{\mu \nu} \nabla_{\mu} A_{\nu}-m \alpha \varphi\right)^{2}\right]
$$

with $\alpha$ being an arbitrary gauge-fixing parameter. The gauge-fixed Stueckelberg action then splits into two sectors, the scalar one,

$$
S_{*}^{\varphi}[\varphi]=\int d^{D} x \sqrt{-g}\left[-\frac{1}{2} g^{\mu \nu}\left(\partial_{\mu} \varphi\right)\left(\partial_{\nu} \varphi\right)-\frac{\alpha m^{2}}{2} \varphi^{2}\right],
$$

and the vector one,

$$
S_{*}^{A}\left[A_{\mu}\right]=\int d^{D} x \sqrt{-g}\left[-\frac{1}{4} g^{\mu \rho} g^{\nu \sigma} F_{\mu \nu} F_{\rho \sigma}-\frac{m^{2}}{2} g^{\mu \nu} A_{\mu} A_{\nu}-\frac{1}{2 \alpha}\left(g^{\mu \nu} \nabla_{\mu} A_{\nu}\right)^{2}\right] .
$$

It is this vector sector gauge-fixed action that was taken as a starting point in [79]. The gauge-fixing procedure above explains the origin of this action, and why it can contain both 
the mass term and the gauge-fixing term for the vector field. The Stueckelberg theory in de Sitter is adequate to work out the propagators. However, one must bear in mind that it is a linear theory, and that if the propagators are to be used in loop computations, the vertices of the interacting gauge theory have to be worked out carefully, as we do for the Abelian Higgs model in the unitary gauge is section 3.

Fixing the unitary gauge in the Stueckelberg model via the Dirac-Bergmann algorithm of section 3.1 would amount to fixing $\varphi=0$. However, it is a common lore that the limit $\alpha \rightarrow \infty$ of the $R_{\xi}$ gauge reproduces the unitary gauge by making the scalar in (7.102) infinitely massive. What is in fact shown in [79] is that this limit reproduces only the covariant Green's function, but not the Feynman propagator.

Another noteworthy result for the vector field propagator was reported by Tsamis and Woodard [78], and is the only one out of the three commented on in this section which has been successfully employed in loop computations in de Sitter space [27, 50, 81-84]. Ref. [78] reported the propagator in the covariant Landau gauge (exact covariant gauge), which corresponds to the $\alpha \rightarrow 0$ of the $R_{\xi}$ gauge-fixing functional, and is equally well adapted to studying both massless and massive vector fields. We cannot compare our propagator to theirs, as the Landau and unitary gauges correspond to the opposite limits of the $R_{\xi}$ gauge.

\section{Field strength correlator}

In this section we make use of the vector field two-point function to compute a simple observable - the off-coincident correlator of the vector field strength tensor $F_{\mu \nu}$, that is of central importance in primordial magnetogenesis scenarios (see e.g. [39-41] for recent reviews). It can be expressed in terms of derivative operators acting on the two-point function,

$$
\left\langle\Omega\left|\hat{F}_{\mu \nu}(x) \hat{F}_{\rho \sigma}\left(x^{\prime}\right)\right| \Omega\right\rangle=4\left(\partial_{[\mu} \delta_{\nu]}^{\alpha}\right)\left(\partial_{[\rho}^{\prime} \delta_{\sigma]}^{\beta}\right) i\left[{ }_{\alpha}^{-} \Delta_{\beta}^{+}\right]\left(x ; x^{\prime}\right)
$$

where $i\left[{ }_{\alpha}^{-} \Delta_{\beta}^{+}\right]\left(x ; x^{\prime}\right)$ is the Wightman function for the vector potential given by (7.40), with scalar structure functions in (7.47)-(7.50). This correlator is gauge invariant, due to the vector field strength tensor being gauge invariant itself, and it takes the same form in all gauges. It can be expanded in the appropriate tensor basis, taking into account the anti-symmetrization of indices,

$$
\begin{aligned}
\langle\Omega| \hat{F}_{\mu \nu}(x) & \hat{F}_{\rho \sigma}\left(x^{\prime}\right)|\Omega\rangle=\left(\partial_{\mu} \partial_{[\rho}^{\prime} y_{-+}\right)\left(\partial_{\sigma]}^{\prime} \partial_{\nu} y_{-+}\right) \times \mathcal{K}_{1}\left(y_{-+}, u\right) \\
& +\left(\partial_{[\mu} y_{-+}\right)\left(\partial_{\nu]} \partial_{[\sigma}^{\prime} y_{-+}\right)\left(\partial_{\rho]}^{\prime} y_{-+}\right) \times \mathcal{K}_{2}\left(y_{-+}, u\right) \\
& +\left[\left(\partial_{[\mu} y_{-+}\right)\left(\partial_{\nu]} \partial_{[\sigma}^{\prime} y_{-+}\right)\left(\partial_{\rho]}^{\prime} u\right)+\left(\partial_{[\mu} u\right)\left(\partial_{\nu]} \partial_{[\sigma}^{\prime} y_{-+}\right)\left(\partial_{\rho]}^{\prime} y_{-+}\right)\right] \times \mathcal{K}_{3}\left(y_{-+}, u\right) \\
& +\left(\partial_{[\mu} u\right)\left(\partial_{\nu]} \partial_{[\sigma}^{\prime} y_{-+}\right)\left(\partial_{\rho]}^{\prime} u\right) \times \mathcal{K}_{4}\left(y_{-+}, u\right) \\
& +\left(\partial_{[\mu} y_{-+}\right)\left(\partial_{\nu]} u\right)\left(\partial_{[\rho}^{\prime} y_{-+}\right)\left(\partial_{\sigma]}^{\prime} u\right) \times \mathcal{K}_{5}\left(y_{-+}, u\right)
\end{aligned}
$$


where the scalar structure functions $\mathcal{K}_{i}$ 's of this expansion are expressed in terms of the scalar structure functions $\mathcal{C}_{i}$ 's of the vector potential two-point functions,

$$
\begin{aligned}
& \mathcal{K}_{1}(y, u)=4\left(\frac{\partial \mathcal{C}_{1}}{\partial y}-\mathcal{C}_{2}\right) \\
& \mathcal{K}_{2}(y, u)=4 \frac{\partial}{\partial y}\left(\frac{\partial \mathcal{C}_{1}}{\partial y}-\mathcal{C}_{2}\right)=\frac{\partial \mathcal{K}_{1}}{\partial y} \\
& \mathcal{K}_{3}(y, u)=4 \frac{\partial}{\partial u}\left(\frac{\partial \mathcal{C}_{1}}{\partial y}-\mathcal{C}_{2}\right)=\frac{\partial \mathcal{K}_{1}}{\partial u} \\
& \mathcal{K}_{4}(y, u)=4\left(\frac{\partial^{2} \mathcal{C}_{1}}{\partial u^{2}}-2 \frac{\partial \mathcal{C}_{3}}{\partial u}+\frac{\partial \mathcal{C}_{4}}{\partial y}\right) \\
& \mathcal{K}_{5}(y, u)=4\left(\frac{\partial^{2} \mathcal{C}_{2}}{\partial u^{2}}-2 \frac{\partial^{2} \mathcal{C}_{3}}{\partial y \partial u}+\frac{\partial^{2} \mathcal{C}_{4}}{\partial y^{2}}\right)=-\frac{\partial^{2} \mathcal{K}_{1}}{\partial u^{2}}+\frac{\partial \mathcal{K}_{4}}{\partial y}
\end{aligned}
$$

The relations above are simply derived by acting the derivatives in (8.1) onto the covariantized Wightman function in (7.40), and exploiting the anti-symmetrization of derivatives. We then plug in the $\mathcal{C}_{i}$ 's from (7.47)-(7.50) into the structure functions above, and use the hypergeometric equation (B.4) to express them in terms of the rescaled propagator function,

$$
\begin{aligned}
& \mathcal{K}_{1}(y, u)=\frac{2 e^{-\frac{(D-4) \epsilon}{2(1-\epsilon)} u}}{(1-\epsilon)^{2} H_{0}^{2}} \times\left[-\frac{\partial}{\partial y} \mathcal{F}_{\nu}(y)\right] \\
& \mathcal{K}_{2}(y, u)=\frac{2 e^{-\frac{(D-4) \epsilon}{2(1-\epsilon)} u}}{(1-\epsilon)^{2} H_{0}^{2}} \times\left[-\frac{\partial^{2}}{\partial y^{2}} \mathcal{F}_{\nu}(y)\right] \\
& \mathcal{K}_{3}(y, u)=\frac{2 e^{-\frac{(D-4) \epsilon}{2(1-\epsilon)} u}}{(1-\epsilon)^{2} H_{0}^{2}} \times\left[\frac{(D-4) \epsilon}{2(1-\epsilon)} \frac{\partial}{\partial y} \mathcal{F}_{\nu}(y)\right] \\
& \mathcal{K}_{4}(y, u)=\frac{2 e^{-\frac{(D-4) \epsilon}{2(1-\epsilon)} u}}{(1-\epsilon)^{2} H_{0}^{2}} \times\left[\frac{(D-4) \epsilon}{2(1-\epsilon)}\left(1-\frac{(D-4) \epsilon}{2(1-\epsilon)}\right) \mathcal{F}_{\nu}(y)\right] \\
& \mathcal{K}_{5}(y, u)=\frac{2 e^{-\frac{(D-4) \epsilon}{2(1-\epsilon)} u}}{(1-\epsilon)^{2} H_{0}^{2}} \times\left[\frac{(D-4) \epsilon}{2(1-\epsilon)} \frac{\partial}{\partial y} \mathcal{F}_{\nu}(y)\right]
\end{aligned}
$$

These structure functions, together with the tensor basis expansion (8.2) represent our result for the vector field strength correlator in the Abelian Higgs model in $D$-dimensional power-law inflation.

It is worth noting that all the scalar structure functions (8.8)-(8.12) of the vector field strength correlator are regular in the limit of vanishing condensate, despite the fact that this is a singular point of the unitary gauge. They only depend on the condensate via the index of the rescaled propagator functions, which simply reduces to $\nu \stackrel{\bar{\phi}_{0} \rightarrow 0}{\longrightarrow}(D-3-$ $\epsilon) /[2(1-\epsilon)]$, introducing no singularities into the correlator. The fact that the correlator in (8.2) and (8.8)-(8.12) is regular for $\bar{\phi} \rightarrow 0$ is not obvious. The issue is clarified by writing out the components of the correlator in terms of the transverse and longitudinal two-point 
functions computed in section 7.3 ,

$$
\begin{aligned}
\left\langle\Omega\left|\hat{F}_{0 i}(x) \hat{F}_{0 j}\left(x^{\prime}\right)\right| \Omega\right\rangle= & \partial_{0} \partial_{0}^{\prime}\left\langle\Omega\left|\hat{A}_{i}^{\mathrm{T}}(x) \hat{A}_{j}^{\mathrm{T}}\left(x^{\prime}\right)\right| \Omega\right\rangle \\
& +\left(a a^{\prime}\right)^{4-D}\left\langle\Omega\left|\hat{\Pi}_{i}^{\mathrm{L}}(x) \hat{\Pi}_{j}^{\mathrm{L}}\left(x^{\prime}\right)\right| \Omega\right\rangle, \\
\left\langle\Omega\left|\hat{F}_{0 i}(x) \hat{F}_{k l}\left(x^{\prime}\right)\right| \Omega\right\rangle= & 2 \partial_{k]}^{\prime} \partial_{0}\left\langle\Omega\left|\hat{A}_{i}^{\mathrm{T}}(x) \hat{A}_{[l}^{\mathrm{T}}\left(x^{\prime}\right)\right| \Omega\right\rangle, \\
\left\langle\Omega\left|\hat{F}_{i j}(x) \hat{F}_{k l}\left(x^{\prime}\right)\right| \Omega\right\rangle= & 4 \partial_{k]}^{\prime} \partial_{[i}\left\langle\Omega\left|\hat{A}_{j]}^{\mathrm{T}}(x) \hat{A}_{[l}^{\mathrm{T}}\left(x^{\prime}\right)\right| \Omega\right\rangle .
\end{aligned}
$$

It is clear that both the longitudinal and the transverse components contribute to the correlators in general. It is also clear that the contribution of the longitudinal component drops out in the limit of vanishing condensate, as the correlator of longitudinal momenta is suppressed by the condensate parameter $q \bar{\phi}_{0} / H_{0}$ as seen from (7.28). Thus the massless vector field limit is recovered, where only the spatially transverse vector potential contributes to the vector field strength. In fact, in the limit of vanishing condensate in $D=4$ space-time dimensions our correlator reduces to the flat space result for the massless $\mathrm{U}(1)$ vector field,

$$
\left\langle\Omega\left|\hat{F}_{\mu \nu}(x) \hat{F}_{\rho \sigma}\left(x^{\prime}\right)\right| \Omega\right\rangle \underset{D \rightarrow 4}{\stackrel{\bar{\phi}_{0} \rightarrow 0}{\longrightarrow}} \frac{2}{\pi^{2}\left(\Delta x^{2}\right)^{2}}\left[\eta_{\mu[\rho} \eta_{\sigma] \nu}-4 \eta_{\alpha[\mu} \eta_{\nu][\sigma} \eta_{\rho] \beta} \frac{\Delta x^{\alpha} \Delta x^{\beta}}{\Delta x^{2}}\right] .
$$

This is so because the massless $\mathrm{U}(1)$ vector field is coupled conformally to gravity in $D=4$. This property of regularity in the limit of vanishing condensate can be understood by recalling that the field strength correlator is a gauge invariant quantity, and hence it must be identical in all gauges, regardless whether they are singular or not.

\subsection{Equal time E\&M correlators}

In the context of primordial magnetogenesis (and electrogenesis), the relevant vector field strength correlators are the equal-time electric and magnetic (E\&M) field correlators in $D=4$ space-time dimensions. The electric and magnetic field operators are given as the following components of the vector field strength operators,

$$
\hat{E}_{i}=\hat{F}_{0 i}, \quad \hat{B}_{i}=\frac{1}{2} \varepsilon_{i j k} \hat{F}_{j k},
$$

where $\varepsilon_{i j k}$ is the 3 -dimensional Levi-Civita symbol, so that their equal-time correlators can be expressed in terms of the equal-time correlators of the vector field strength (8.2),

$$
\begin{aligned}
\left\langle\Omega\left|\hat{E}_{i}(\eta, \vec{x}) \hat{E}_{j}\left(\eta, \vec{x}^{\prime}\right)\right| \Omega\right\rangle & =\left\langle\Omega\left|\hat{F}_{0 i}(\eta, \vec{x}) \hat{F}_{0 j}\left(\eta, \vec{x}^{\prime}\right)\right| \Omega\right\rangle, \\
\left\langle\Omega\left|\hat{E}_{i}(\eta, \vec{x}) \hat{B}_{j}\left(\eta, \vec{x}^{\prime}\right)\right| \Omega\right\rangle & =\frac{1}{2} \varepsilon_{j k l}\left\langle\Omega\left|\hat{F}_{0 i}(\eta, \vec{x}) \hat{F}_{k l}\left(\eta, \vec{x}^{\prime}\right)\right| \Omega\right\rangle, \\
\left\langle\Omega\left|\hat{B}_{i}(\eta, \vec{x}) \hat{B}_{j}\left(\eta, \vec{x}^{\prime}\right)\right| \Omega\right\rangle & =\frac{1}{4} \varepsilon_{i k l} \varepsilon_{j m n}\left\langle\Omega\left|\hat{F}_{k l}(\eta, \vec{x}) \hat{F}_{m n}\left(\eta, \vec{x}^{\prime}\right)\right| \Omega\right\rangle .
\end{aligned}
$$

The last three scalar structure functions of the vector field strength correlator (8.2) drop out in $D=4$. Therefore, we only need the equal-time limit of the first two tensor structures, computed using expressions (C.2)-(C.8) for derivatives of the distance function,

$$
\begin{aligned}
&\left(\partial_{0} \partial_{[0}^{\prime} y\right)\left(\partial_{j]}^{\prime} \partial_{i} y\right) \stackrel{\eta^{\prime} \rightarrow \eta}{\longrightarrow}[(1-\epsilon) \mathcal{H}]^{4}\left[-\left(\mathcal{Y}^{2}+2\right) \delta_{i j}+2 \mathcal{Y}^{2} \frac{\Delta x_{i} \Delta x_{j}}{\|\Delta \vec{x}\|^{2}}\right], \\
& \frac{1}{2} \varepsilon_{j k l}\left(\partial_{0} \partial_{[k}^{\prime} y\right)\left(\partial_{l]}^{\prime} \partial_{i} y\right) \stackrel{\eta^{\prime} \rightarrow \eta}{\longrightarrow}[(1-\epsilon) \mathcal{H}]^{4}\left[2 \mathcal{Y} \varepsilon_{i j k} \frac{\Delta x_{k}}{\|\Delta \vec{x}\|}\right],
\end{aligned}
$$




$$
\begin{gathered}
\frac{1}{4} \varepsilon_{i k l} \varepsilon_{j m n}\left(\partial_{k} \partial_{[m}^{\prime} y\right)\left(\partial_{n]}^{\prime} \partial_{l} y\right) \stackrel{\eta^{\prime} \rightarrow \eta}{\longrightarrow}[(1-\epsilon) \mathcal{H}]^{4}\left[2 \delta_{i j}\right] \\
\left(\partial_{[0} y\right)\left(\partial_{i]} \partial_{[j}^{\prime} y\right)\left(\partial_{0]}^{\prime} y\right) \stackrel{\eta^{\prime} \rightarrow \eta}{\longrightarrow}[(1-\epsilon) \mathcal{H}]^{4}\left[-\frac{\mathcal{Y}^{4}}{2} \delta_{i j}+\mathcal{Y}^{2}\left(\mathcal{Y}^{2}-2\right) \frac{\Delta x_{i} \Delta x_{j}}{\|\Delta \vec{x}\|^{2}}\right] \\
\frac{1}{2} \varepsilon_{j k l}\left(\partial_{[0} y\right)\left(\partial_{i]} \partial_{[l}^{\prime} y\right)\left(\partial_{k]}^{\prime} y\right) \stackrel{\eta^{\prime} \rightarrow \eta}{\longrightarrow}[(1-\epsilon) \mathcal{H}]^{4}\left[\mathcal{Y}^{3} \varepsilon_{i j k} \frac{\Delta x_{k}}{\|\Delta \vec{x}\|}\right] \\
\frac{1}{4} \varepsilon_{i k l} \varepsilon_{j m n}\left(\partial_{[k} y\right)\left(\partial_{l]} \partial_{[n}^{\prime} y\right)\left(\partial_{m]}^{\prime} y\right) \stackrel{\eta^{\prime} \rightarrow \eta}{\longrightarrow}[(1-\epsilon) \mathcal{H}]^{4}\left[2 \mathcal{Y}^{2} \delta_{i j}-2 \mathcal{Y}^{2} \frac{\Delta x_{i} \Delta x_{j}}{\|\Delta \vec{x}\|^{2}}\right]
\end{gathered}
$$

where for convenience we define the quantity $\mathcal{Y}$ as a square root of the equal-time limit of the distance function,

$$
y \stackrel{\eta^{\prime} \rightarrow \eta}{\longrightarrow}[(1-\epsilon) \mathcal{H}\|\Delta \vec{x}\|]^{2} \equiv \mathcal{Y}^{2},
$$

and the equal-time limit of the rescaled propagator function,

$$
\frac{\mathcal{F}_{\nu}(y)}{\left[(1-\epsilon) H_{0}\right]^{2}} \stackrel{\eta^{\prime} \rightarrow \eta}{\longrightarrow} \frac{\Gamma\left(\frac{3}{2}+\nu\right) \Gamma\left(\frac{3}{2}-\nu\right)}{(4 \pi)^{2}}{ }_{2} F_{1}\left(\left\{\frac{3}{2}+\nu, \frac{3}{2}-\nu\right\},\{2\}, 1-\frac{1}{4} \mathcal{Y}^{2}\right) \equiv \mathscr{F}_{\nu}(\mathcal{Y}),
$$

where we have defined a dimensionless function $\mathscr{F}_{\nu}$, and where the index $\nu$ is evaluated in $D=4$,

$$
\nu \stackrel{D \rightarrow 4}{\longrightarrow} \sqrt{\frac{1}{4}-\left(\frac{q \bar{\phi}_{0}}{(1-\epsilon) H_{0}}\right)^{2}}, \quad\left(\frac{q \bar{\phi}_{0}}{H_{0}}\right)^{2}=\frac{q^{2}}{\lambda}[\epsilon(3-2 \epsilon)-6 \xi(2-\epsilon)]>0 .
$$

Furthermore, the partial derivatives appearing in the structure functions are expressed as,

$$
\frac{\partial}{\partial y} \stackrel{\eta^{\prime} \rightarrow \eta}{\longrightarrow} \frac{1}{2(1-\epsilon)^{2} \mathcal{H}^{2}\|\Delta \vec{x}\|} \frac{\partial}{\partial\|\Delta \vec{x}\|}=\frac{1}{2 \mathcal{Y}} \frac{\partial}{\partial \mathcal{Y}} .
$$

This allows us to write the equal-time electric and magnetic field correlators as,

$$
\begin{aligned}
\langle\Omega| \hat{E}_{i}(\eta, \vec{x}) & \hat{E}_{j}\left(\eta, \vec{x}^{\prime}\right)|\Omega\rangle \\
= & \frac{\mathcal{Y}^{4}}{\|\Delta \vec{x}\|^{4}}\left\{\delta_{i j}\left[\frac{2}{\mathcal{Y}}+\frac{3 \mathcal{Y}}{4}+\frac{\mathcal{Y}^{2}}{4} \frac{\partial}{\partial \mathcal{Y}}\right]\right. \\
& \left.+\left(\frac{\Delta x_{i} \Delta x_{j}}{\|\Delta \vec{x}\|^{2}}\right)\left[-\frac{1}{\mathcal{Y}}-\frac{3 \mathcal{Y}}{2}+\left(1-\frac{\mathcal{Y}^{2}}{2}\right) \frac{\partial}{\partial \mathcal{Y}}\right]\right\} \frac{\partial}{\partial \mathcal{Y}} \mathscr{F}_{\nu}(\mathcal{Y}), \\
\langle\Omega| \hat{E}_{i}(\eta, \vec{x}) & \hat{B}_{j}\left(\eta, \vec{x}^{\prime}\right)|\Omega\rangle \\
= & \frac{\mathcal{Y}^{4}}{\|\Delta \vec{x}\|^{4}}\left\{\left(\varepsilon_{i j k} \frac{\Delta x_{k}}{\|\Delta \vec{x}\|}\right)\left[-\frac{3}{2}-\frac{\mathcal{Y}}{2} \frac{\partial}{\partial \mathcal{Y}}\right]\right\} \frac{\partial}{\partial \mathcal{Y}} \mathscr{F}_{\nu}(\mathcal{Y}), \\
\langle\Omega| \hat{B}_{i}(\eta, \vec{x}) & \hat{B}_{j}\left(\eta, \vec{x}^{\prime}\right)|\Omega\rangle \\
= & \frac{\mathcal{Y}^{4}}{\|\Delta \vec{x}\|^{4}}\left\{\delta_{i j}\left[-\frac{1}{\mathcal{Y}}-\frac{\partial}{\partial \mathcal{Y}}\right]+\left(\frac{\Delta x_{i} \Delta x_{j}}{\|\Delta \vec{x}\|^{2}}\right)\left[-\frac{1}{\mathcal{Y}}+\frac{\partial}{\partial \mathcal{Y}}\right]\right\} \frac{\partial}{\partial \mathcal{Y}} \mathscr{F}_{\nu}(\mathcal{Y}),
\end{aligned}
$$

where we rewrote the overall factor as $[(1-\epsilon) \mathcal{H}]^{4}=\mathcal{Y}^{4} /\|\Delta \vec{x}\|^{4}$ for later convenience. 


\subsubsection{Sub-Hubble limit}

The sub-Hubble limit is defined by $\mathcal{Y}=(1-\epsilon) \mathcal{H}\|\Delta \vec{x}\| \ll 1$, which we denote by $\stackrel{\text { sH }}{\sim}$. In this limit the function $\mathscr{F}_{\nu}(\mathcal{Y})$ from (8.28) reduces to (cf. eq. 7.57),

$$
\mathscr{F}_{\nu}(\mathcal{Y}) \stackrel{\text { sH }}{\sim} \frac{1}{\pi^{2} \mathcal{Y}^{2}},
$$

so that the electric and magnetic correlators (8.31)-(8.33) at leading order reduce to

$$
\begin{aligned}
& \left\langle\Omega\left|\hat{E}_{i}(\eta, \vec{x}) \hat{E}_{j}\left(\eta, \vec{x}^{\prime}\right)\right| \Omega\right\rangle \stackrel{\mathrm{sH}}{\sim} \frac{1}{\pi^{2}\|\Delta \vec{x}\|^{4}}\left[-\delta_{i j}+2\left(\frac{\Delta x_{i} \Delta x_{j}}{\|\Delta \vec{x}\|^{2}}\right)+\mathcal{O}\left(\mathcal{Y}^{2}\right)\right], \\
& \left\langle\Omega\left|\hat{E}_{i}(\eta, \vec{x}) \hat{B}_{j}\left(\eta, \vec{x}^{\prime}\right)\right| \Omega\right\rangle \stackrel{\mathrm{sH}}{\sim} \frac{1}{\pi^{2}\|\Delta \vec{x}\|^{4}}\left[0+\mathcal{O}\left(\mathcal{Y}^{2}\right)\right], \\
& \left\langle\Omega\left|\hat{B}_{i}(\eta, \vec{x}) \hat{B}_{j}\left(\eta, \vec{x}^{\prime}\right)\right| \Omega\right\rangle \stackrel{\mathrm{sH}}{\sim} \frac{1}{\pi^{2}\|\Delta \vec{x}\|^{4}}\left[-\delta_{i j}+2\left(\frac{\Delta x_{i} \Delta x_{j}}{\|\Delta \vec{x}\|^{2}}\right)+\mathcal{O}\left(\mathcal{Y}^{2}\right)\right] .
\end{aligned}
$$

Interestingly, these match the E\&M correlators of a massless vector field. ${ }^{18}$

\subsubsection{Super-Hubble limit}

The interesting limit of the E\&M correlators is the super-Hubble limit defined by $\mathcal{Y}=$ $(1-\epsilon) \mathcal{H}\|\Delta \vec{x}\| \gg 1$, which we denote is by $\stackrel{\mathrm{SH}}{\sim}$. In this limit the asymptotic behaviour of the function $\mathscr{F}_{\nu}$ is given by the equal-time limit and the $D=4$ limit of the asymptotic expansion (B.8) from appendix B,

$$
\mathscr{F}_{\nu}(\mathcal{Y}) \stackrel{\mathrm{SH}}{\sim} \frac{\Gamma(\nu) \Gamma\left(\frac{3}{2}-\nu\right)}{(2 \pi)^{2} \sqrt{\pi}} \mathcal{Y}^{-3+2 \nu}+\frac{\Gamma(-\nu) \Gamma\left(\frac{3}{2}+\nu\right)}{(2 \pi)^{2} \sqrt{\pi}} \mathcal{Y}^{-3-2 \nu}
$$

where we have used the Legendre duplication formula to simplify the coefficients. We remind the reader that the index $\nu$ given in (8.29) either takes real values $0 \leq \nu<1 / 2$, or is imaginary, depending on the value of the condensate parameter $q \bar{\phi}_{0} / H_{0}$. In case of $\nu>0$ the first term in the asymptotic expansion (8.38) dominates over the second one. However, as $\nu$ approaches 0 , the two become of the same order. For imaginary $\nu$ the two terms are exactly of the same order, both scaling as $\mathcal{Y}^{-3}$, and they pick up an overall oscillating factor with a logarithmically growing frequency,

$$
\mathscr{F}_{i|\nu|}(\mathcal{Y}) \stackrel{\mathrm{SH}}{\sim} 2 \operatorname{Re}\left[\frac{\Gamma(i|\nu|) \Gamma\left(\frac{3}{2}-i|\nu|\right)}{(2 \pi)^{2} \sqrt{\pi}} e^{2 i|\nu| \ln (\mathcal{Y})}\right] \mathcal{Y}^{-3}
$$

Thus, the asymptotic form in (8.38) captures the leading order super-Hubble behaviour in all cases. Using it in (8.31)-(8.33) captures the leading order super-Hubble behaviour of

\footnotetext{
${ }^{18}$ The limiting form in (8.34) is correct as long as $\mathcal{Y}<1 /|\nu|$ in addition to $\mathcal{Y} \ll 1$. If that is not satisfied, the proper sub-Hubble asymptotic behaviour is captured by the $|\nu| \gg 1$ expansion of $\mathscr{F}_{\nu}(\mathcal{Y})$, which corresponds to $(7.76)$ with the substitution $m^{2} \rightarrow\left(q \bar{\phi}_{0}\right)^{2}$. This limit requires $\left(q \bar{\phi}_{0}\right)^{2} \gg H_{0}^{2}$, which is possible for very large and negative non-minimal coupling $\xi$, or for very small quartic self-coupling $\lambda$. We do not consider these cases here.
} 
E\&M correlators,

$$
\begin{aligned}
& \left\langle\Omega\left|\hat{E}_{i}(\eta, \vec{x}) \hat{E}_{j}\left(\eta, \vec{x}^{\prime}\right)\right| \Omega\right\rangle \\
& \stackrel{\mathrm{SH}}{\sim} \frac{\mathcal{Y}}{\pi^{2}\|\Delta \vec{x}\|^{4}}\left[\frac{\left(\frac{1}{2}-\nu\right) \Gamma(\nu) \Gamma\left(\frac{5}{2}-\nu\right)}{4 \sqrt{\pi}} \mathcal{Y}^{2 \nu}\right. \\
& \left.+\frac{\left(\frac{1}{2}+\nu\right) \Gamma(-\nu) \Gamma\left(\frac{5}{2}+\nu\right)}{4 \sqrt{\pi}} \mathcal{Y}^{-2 \nu}\right]\left[\delta_{i j}-2\left(\frac{\Delta x_{i} \Delta x_{j}}{\|\Delta \vec{x}\|^{2}}\right)\right], \\
& \left\langle\Omega\left|\hat{E}_{i}(\eta, \vec{x}) \hat{B}_{j}\left(\eta, \vec{x}^{\prime}\right)\right| \Omega\right\rangle, \\
& \stackrel{\mathrm{SH}}{\sim} \frac{1}{\pi^{2}\|\Delta \vec{x}\|^{4}}\left[-\frac{\left(\frac{1}{2}-\nu\right) \Gamma(\nu) \Gamma\left(\frac{5}{2}-\nu\right)}{2 \sqrt{\pi}} \mathcal{Y}^{2 \nu}\right. \\
& \left.-\frac{\left(\frac{1}{2}+\nu\right) \Gamma(-\nu) \Gamma\left(\frac{5}{2}+\nu\right)}{2 \sqrt{\pi}} \mathcal{Y}^{-2 \nu}\right]\left(\varepsilon_{i j k} \frac{\Delta x_{k}}{\|\Delta \vec{x}\|}\right), \\
& \left\langle\Omega\left|\hat{B}_{i}(\eta, \vec{x}) \hat{B}_{j}\left(\eta, \vec{x}^{\prime}\right)\right| \Omega\right\rangle \\
& \stackrel{\mathrm{SH}}{\sim} \frac{\mathcal{Y}^{-1}}{\pi^{2}\|\Delta \vec{x}\|^{4}}\left[-\frac{\left(\frac{3}{2}-\nu\right) \Gamma(\nu) \Gamma\left(\frac{5}{2}-\nu\right)}{\sqrt{\pi}} \mathcal{Y}^{2 \nu}-\frac{\left(\frac{3}{2}+\nu\right) \Gamma(-\nu) \Gamma\left(\frac{5}{2}+\nu\right)}{\sqrt{\pi}} \mathcal{Y}^{-2 \nu}\right] \delta_{i j} \\
& +\frac{\mathcal{Y}^{-1}}{\pi^{2}\|\Delta \vec{x}\|^{4}}\left[\frac{\Gamma(\nu) \Gamma\left(\frac{7}{2}-\nu\right)}{\sqrt{\pi}} \mathcal{Y}^{2 \nu}+\frac{\Gamma(-\nu) \Gamma\left(\frac{7}{2}+\nu\right)}{\sqrt{\pi}} \mathcal{Y}^{-2 \nu}\right]\left(\frac{\Delta x_{i} \Delta x_{j}}{\|\Delta \vec{x}\|^{2}}\right) \text {. }
\end{aligned}
$$

It is clear from the overall factors of the expressions above that the $E E$ correlator at superHubble separations is enhanced compared to the conformal one, while the $B B$ correlator is suppressed. Therefore, the effect of the scalar condensate is to enhance the electric field, and to screen the magnetic field, compared to the case of vanishing condensate (the symmetric phase). Furthermore, it is also clear from the overall factors above that the E\&M correlators satisfy a hierarchy at super-Hubble separations, recently reported for power-law inflation in [7], which reads (up to numerical factors),

$$
\begin{aligned}
&\left\langle\hat{E}_{i}(\eta, \vec{x}) \hat{E}_{j}\left(\eta, \vec{x}^{\prime}\right)\right\rangle /\left\langle\hat{E}_{i}(\eta, \vec{x}) \hat{B}_{j}\left(\eta, \vec{x}^{\prime}\right)\right\rangle /\left\langle\hat{B}_{i}(\eta, \vec{x})\right.\left.\hat{B}_{j}\left(\eta, \vec{x}^{\prime}\right)\right\rangle \\
& \stackrel{\mathrm{SH}}{\sim}(\mathcal{H}\|\Delta \vec{x}\|)^{2} / \mathcal{H}\|\Delta \vec{x}\| / 1 .
\end{aligned}
$$

The scaling of the ratios above is a consequence of Faraday's law in expanding space-times, as reported in [106], and does not depend on the sources. The hierarchy between the correlators develops dynamically in accelerating space-times.

It is instructive to examine appropriately contracted correlators,

$$
\begin{aligned}
\delta_{i j}\left\langle\Omega\left|\hat{E}_{i}(\eta, \vec{x}) \hat{E}_{j}\left(\eta, \vec{x}^{\prime}\right)\right| \Omega\right\rangle & =-\frac{1}{\pi^{2}\|\Delta \vec{x}\|^{4}} \times E E(\mathcal{Y}), \\
\frac{\Delta x_{k}}{\|\Delta \vec{x}\|} \varepsilon_{k i j}\left\langle\Omega\left|\hat{B}_{i}(\eta, \vec{x}) \hat{E}_{i}\left(\eta, \vec{x}^{\prime}\right)\right| \Omega\right\rangle & =-\frac{1}{\pi^{2}\|\Delta \vec{x}\|^{4}} \times E B(\mathcal{Y}), \\
\delta_{i j}\left\langle\Omega\left|\hat{B}_{i}(\eta, \vec{x}) \hat{B}_{j}\left(\eta, \vec{x}^{\prime}\right)\right| \Omega\right\rangle & =-\frac{1}{\pi^{2}\|\Delta \vec{x}\|^{4}} \times B B(\mathcal{Y}),
\end{aligned}
$$

normalized to the conformal correlators $\left\langle\hat{E}_{i}(\eta, \vec{x}) \hat{E}_{i}\left(\eta, \vec{x}^{\prime}\right)\right\rangle_{\mathrm{cf}}=\left\langle\hat{B}_{i}(\eta, \vec{x}) \hat{B}_{i}\left(\eta, \vec{x}^{\prime}\right)\right\rangle_{\mathrm{cf}}=$ $-1 /\left(\pi^{2}\|\Delta \vec{x}\|^{4}\right)$, i.e. the E\&M correlators of a massless vector field. They are plotted in 

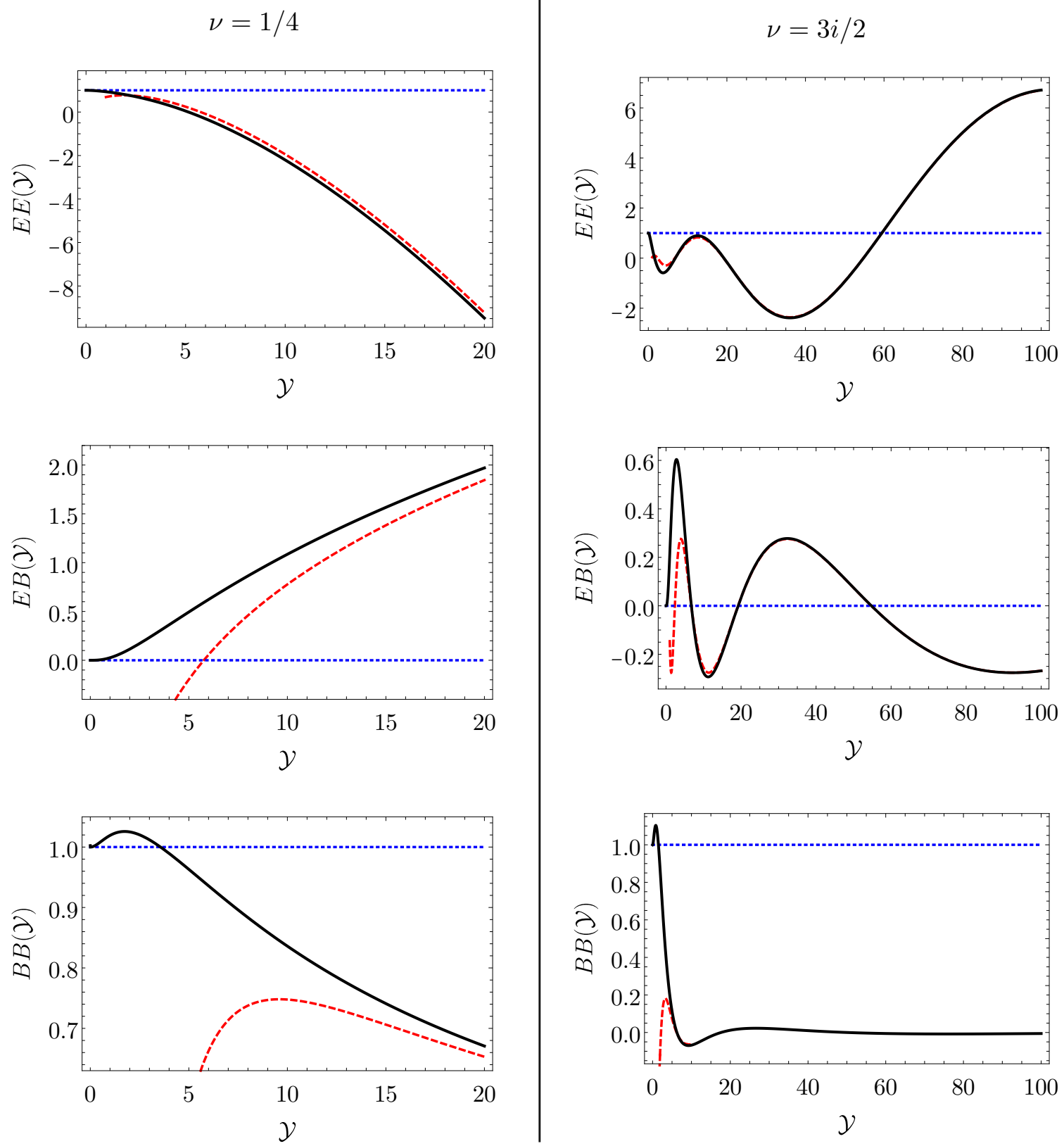

Figure 2. Plots of the contracted E\&M correlators (8.44)-(8.46), rescaled by the contracted conformal correlator for two cases: $\nu=1 / 4$ (left), and $\nu=3 i / 2$ (right). The solid black lines represents the full contracted correlators, dashed red lines are the corresponding super-Hubble asymptotic forms, and dotted blue lined denote the conformal correlators. 
figure 2 for two cases of real and imaginary $\nu$. Note that the $E E$ correlator is in general enhanced at super-Hubble separations compared to the conformal one, the $E B$ exhibits at best a mild growth, and the $B B$ correlator is suppressed compared to the conformal value. At sub-Hubble separations all three correlators are negative (as are the conformal ones) signifying anti-correlation of E\&M field fluctuations on those scales. On super-Hubble separations, and for positive $\nu$ only the $E E$ correlator is positive, while the remaining two are negative, while for imaginary $\nu$ they all oscillate between positive and negative values with a logarithmically growing frequency. The appearance of negative correlators is not unusual in quantum field theory, see e.g. ref. [107].

\section{Discussion}

In this paper we consider the spectator Abelian Higgs model in power-law inflation, characterized by a constant principal slow-roll parameter $\epsilon=\dot{H} / H^{2}=$ const., $0 \leq \epsilon<1$ in $D$ space-time dimensions. We impose the unitary gauge and perform canonical quantization, and construct the propagators for the massive scalar and the massive vector fluctuations of the model. We assume that the vector and the scalar masses are generated by a timedependent condensate of the complex scalar field $\bar{\phi}(t)$. This condensate can be a true scalar condensate or it can be composed of super-Hubble scalar field fluctuations, as it is often the case in inflation $[24-27,81,82]$. We assume that the condensate scales as the Hubble parameter, $\bar{\phi}(t) \propto H$, in which case finding the solution for the propagator dramatically simplifies.

For pedagogical reasons we fix the unitary gauge of the Abelian Higgs model by using Dirac's canonical quantization, which is suitable for studying systems with constraints. Even though Dirac's quantization is standard in some other areas of physics, it is rarely used in cosmology, and this justifies our detailed analysis in section 3. The symmetries of the cosmological background naturally select spatial momentum space, in which the analysis reduces to solving the equations of motion for the suitably rescaled mode functions. The details of that procedure are given in section 3.1. Section 6 is devoted to the evolution of the perturbations of the vector mode functions and in section 7 we construct the covariantized two-point functions for the vector field: the Wightman function (7.40), the Feynman propagator (7.55), and the Green's function (7.56), with the scalar structure functions given in (7.47)-(7.50). These constitute the main results of our paper. The distinction between the Feynman propagator and the Green's function for the vector propagator is a particular feature of the unitary gauge, following from the canonical quantization, and has implications for the renormalizability of the theory, which is not well understood in curved space-times. Together with the interaction vertices, these propagators represent the basic building blocks for dimensionally regulated perturbation theory of the Abelian Higgs model in the unitary gauge on accelerating cosmological spaces. ${ }^{19}$

\footnotetext{
${ }^{19}$ One can also use these propagators on decelerating cosmological backgrounds with $\epsilon=$ const. $>1$, as the form of the propagators depends only on the condensate evolution tracing the Hubble rate, $\bar{\phi} \propto H$, which is maintained here. However, one should keep in mind that in decelerating spaces the CTBD state is not preferred, as it is in accelerating spaces based on simple physical arguments presented in section 7.2.
} 
In section 7.5 we check the consistency of our propagator by working out several limits, and verifying that they reproduce the known results, including that it reduces to the correct massive vector propagator in the unitary gauge (7.80) in Minkowski space-time. We pay special attention to the de Sitter limit of the propagator, which has been studied in the literature, and verify that it correctly reproduces the corresponding Allen-Jacobson massive vector propagator [76]. In addition, our propagator also provides the missing $i \varepsilon-$ prescriptions for the tensor structures, responsible for the distinction between the Feynman propagator and the Green's function. We also comment on how the propagators of the gauge-fixed Stueckelberg theory in de Sitter [79] are related to the Abelian Higgs model in the broken phase.

The issue of gauge dependence is often neglected or taken lightly in cosmological studies. The importance of understanding gauge dependence in cosmological loop calculations involving graviton and/or gauge field loops was stressed recently in refs. [112, 113]. In order to understand gauge dependence of some (physical) quantity it is important to work with a proper regularization that respects all of the relevant symmetries of the problem and have available the relevant propagators in a wide class of gauges, or at least in different fixed gauges. This work is a step in that direction.

The vector propagators in the unitary gauge constructed in this work can be used to address various problems in cosmological spaces that involve perturbative loop calculations, such as loop corrections induced by the massive vector perturbations on the cosmological perturbations, on the effective inflaton potential, and on the cosmological background (through quantum backreaction). All of these effects may leave observable imprints not only during inflation, but also in the subsequent radiation and matter eras, thus warranting a further study. Here we utilized the massive vector propagator in section 8 to study the tree-level off-coincident electric and magnetic field correlators at super-Hubble separations during power-law inflation. We find the $E E$ correlators of the massive vector field to be larger than in the massless vector case, while the converse is true for the $B B$ correlators. Furthermore, the E\&M correlators satisfy a hierarchy at super-Hubble separations, consistent with the results obtained from Faraday's law in [7, 105]. When evolved to late times, these correlators may be important for our understanding of the origin of the observed large scale magnetic fields both at the galactic and cluster scales.

The propagators constructed here are, strictly speaking, applicable to the Abelian Higgs model which is (i) a spectator in power-law inflation, $\epsilon=$ const., and (ii) for which the scalar condensate traces the evolution of the Hubble rate $\bar{\phi} \propto H=H_{0} a^{-\epsilon}$. Nevertheless, the assumption of the exact power-law inflationary background can be relaxed under certain conditions, and the validity of the propagators extended to the more general backgrounds of slow-roll inflation, where the scalar condensate is the inflaton driving a slow-roll inflation.

In slow-roll inflation the principal slow-roll parameter $\epsilon=-\dot{H} / H^{2}$ is timedependent, $\dot{\epsilon} \neq 0$, but varies slowly such that its time derivative can be considered a small

On the contrary, the quantum state depends profoundly on the evolution of the Universe preceding the decelerating epoch. Scalar field examples for how to deal with such situations are given in refs. [108-111]. 
parameter,

$$
\epsilon_{2} \equiv \frac{\dot{\epsilon}}{\epsilon H}, \quad\left|\epsilon_{2}\right| \ll 1,
$$

where $\epsilon_{2}$ is the second slow-roll parameter. From the cosmological microwave background measurements [11] we know the scalar spectral index, $n_{s} \simeq-2 \epsilon-\epsilon_{2} \simeq 0.965$, and the tensorto-scalar ratio, $r \simeq 16 \epsilon<0.065$, which imply the following hierarchy,

$$
\epsilon \ll \epsilon_{2} \ll 1
$$

where $\epsilon_{2} \simeq 1-n_{s} \simeq 0.03$. The Hubble rate in slow-roll inflation scales with time as,

$$
H(t)=H_{0} \exp \left[-\int_{t_{0}}^{t} d N\left(t^{\prime}\right) \epsilon\left(t^{\prime}\right)\right]
$$

where $N(t)=\ln [a(t)]\left(N\left(t_{0}\right)=0\right)$ is the number of $e$-foldings. Our propagators can be seen to apply to slow-roll inflation, under certain conditions, in cases when the scalar condensate is a spectator, and when it is the inflaton itself driving the expansion. We comment below on the restrictions in these two cases.

- Spectator scalar condensate. When slow-roll inflation is driven by some other inflaton field, and the Abelian Higgs model is a spectator, its scalar condensate is attracted towards the so-called scaling regime, where it scales as the Hubble rate,

$$
\bar{\phi}(t)=\bar{\phi}_{0} \exp \left[-\int_{t_{0}}^{t} d N\left(t^{\prime}\right) \epsilon\left(t^{\prime}\right)\right]=\frac{\bar{\phi}_{0}}{H_{0}} H(t) .
$$

This is the first condition necessary for our propagators to be valid. The second condition concerns the time-dependence of the Hubble rate, which needs to take the same form as in power-law inflation with the constant $\epsilon$ promoted to a slowly varying one, $H(t) \approx H_{0}[a(t)]^{-\epsilon(t)}$. To see under which conditions this holds, we can make a derivative expansion ${ }^{20}$ in the exponent of (9.3),

$$
H(t) \approx H_{0} \exp \left\{-\epsilon(t) N(t)\left[1-\frac{1}{2} \epsilon_{2}(t) N(t)\right]\right\} .
$$

The first term in the exponent corresponds precisely to the power-law inflation scaling with an adiabatically evolving principal slow-roll parameter. Therefore, our propagators will be applicable as long as the second term in the exponent can be neglected, implying,

$$
\Delta N \ll \frac{2}{\epsilon_{2}} \simeq 60
$$

${ }^{20}$ The derivative expansion is generated by iterating partial integrations of the time derivatives,

$$
\int_{t_{0}}^{t} d N\left(t^{\prime}\right) \epsilon\left(t^{\prime}\right)=\int_{0}^{N(t)} d N^{\prime} \epsilon\left(N^{\prime}\right)=\epsilon(t) N(t)\left[1-\frac{1}{2} N(t) \epsilon_{2}(t)\right]+\frac{1}{2} \int_{0}^{N(t)} d N^{\prime} N^{\prime 2} \frac{d}{d N^{\prime}}\left[\epsilon\left(N^{\prime}\right) \epsilon_{2}\left(N^{\prime \prime}\right)\right],
$$

where the derivative inside the last integral can be expressed in terms of higher slow-roll parameters defined as $(d / d N) \epsilon_{n}=\epsilon_{n+1}$. The last term above is the remainder integral, which can be used to estimate the error of the derivative expansion. This approximation scheme is clearly superior to the one obtained by promoting the Hubble rate into a local function of time in the de Sitter two-point functions. 
Given that currently only $\Delta N \sim 10 e$-foldings of inflation are observable, our propagators can safely be used to study the spectator Abelian Higgs model in slow-roll inflation.

- Inflaton scalar condensate. When slow-roll inflation is driven by a condensate of the Abelian Higgs model, such as Higgs inflation (see [114] and references therein), the condensate scales in time as

$$
\phi(t)=\bar{\phi}_{0}-M_{\mathrm{P}} \int_{t_{0}}^{t} d N\left(t^{\prime}\right) \sqrt{2 \epsilon\left(t^{\prime}\right)},
$$

where $M_{\mathrm{P}}=1 / \sqrt{8 \pi G_{\mathrm{N}}}$. This is clearly not the same scaling as that of the Hubble rate (9.3). This inflaton condensate is what generates the mass of the vector field, and our propagators apply only when it approximately traces the evolution of the Hubble rate. This can be seen to hold for a certain number of $e$-foldings. Making use of the derivative expansion, the condensate evolution can be written as

$$
\bar{\phi}(t) \approx \bar{\phi}_{0}\left\{1-N(t) \frac{\sqrt{2 \epsilon(t)} M_{\mathrm{P}}}{\bar{\phi}_{0}}\left[1-\frac{1}{4} N(t) \epsilon_{2}(t)\right]\right\} .
$$

This will correspond to the scaling of the Hubble rate provided that, in addition to (9.6), the following two conditions hold,

$$
\Delta N \ll \frac{1}{\epsilon}, \quad \bar{\phi}_{0} \approx \frac{2 M_{\mathrm{P}}}{\sqrt{2 \epsilon}} .
$$

The hierarchy (9.2) implies that this first condition is weaker than (9.6) and thus not an additional constraint. The second condition in (9.9) can be put in perspective if one recalls that, for a simple class of potentials, $V \propto \phi^{n}$, the inflaton in the slow roll regime develops field values $\bar{\phi} \simeq n M_{\mathrm{P}} / \sqrt{2 \epsilon}$. The case of the quadratic potential $n=2$ then satisfies the condition (9.9). For general inflationary potentials, the condition on $\bar{\phi}_{0}$ in (9.9) singles out the value of the inflaton condensate around which our approximation applies for the range of $e$-foldings given in (9.6).

Finally, it is worth noting that our propagators may be applicable to vector field inflation driven by a condensate of a nonminimally coupled vector field [93-96, 115-118]. However, since in these models the vector field develops a condensate and thus couples to gravitational perturbations, a more elaborate analysis is needed to establish the precise conditions of applicability of our propagator in this class of models. The canonical quantization methods utilized here should provide useful in such studies.

\section{Acknowledgments}

We are thankful to Gerard 't Hooft for the discussion on the unitary gauge. D. G. is grateful to the Division of Theoretical Physics of Rudjer Bošković Institute in Zagreb, and to the Institute for Theoretical Physics of Utrecht University for their hospitality during the first course of this project. This work was partially supported by the Fonds de la Recherche 
Scientifique - FNRS under Grant IISN 4.4517.08 - Theory of fundamental interactions, and by the D-ITP consortium, a program of the Netherlands Organization for Scientific Research (NWO) that is funded by the Dutch Ministry of Education, Culture and Science (OCW).

\section{A Scalar mode functions in power-law inflation}

The mode function $\mathscr{U}_{\lambda}$ of a non-minimally coupled massless scalar field satisfies the following equation of motion in power-law inflation,

$$
\left[\partial_{0}^{2}+k^{2}-\left(\lambda^{2}-\frac{1}{4}\right)(1-\epsilon)^{2} \mathcal{H}^{2}\right] \mathscr{U}_{\lambda}(\eta, \vec{k})=0,
$$

where $\lambda$ is either purely real, or purely imaginary, and depends on the scalar's non-minimal coupling, and on the slow-roll parameter. This equation is ubiquitous to all massless fields in power-law inflation, including the case of the vector field from section 6.3 and 6.4. It reduces to the Bessel equation upon a simple rescaling, and its general solution,

$$
\mathscr{U}_{\lambda}(\eta, \vec{k})=A(\vec{k}) \times U_{\lambda}(\eta, k)+B(\vec{k}) \times U_{\lambda}^{*}(\eta, k),
$$

is conveniently written in terms of suitably rescaled Hankel function of the first kind,

$$
U_{\lambda}(\eta, k)=e^{\frac{i \pi}{4}(2 \lambda+1)} e^{\frac{-i k}{(1-\epsilon) \mathcal{H}_{0}}} \sqrt{\frac{\pi}{4(1-\epsilon) \mathcal{H}}} H_{\lambda}^{(1)}\left(\frac{k}{(1-\epsilon) \mathcal{H}}\right),
$$

where $A$ and $B$ are constants of integration, and $k=\|\vec{k}\|$. The time-independent normalization of the function $U_{\lambda}$ in (A.3) is chosen for convenience, such that its Wronskian normalization,

$$
U_{\lambda}(\eta, k) \partial_{0} U_{\lambda}^{*}(\eta, k)-U_{\lambda}^{*}(\eta, k) \partial_{0} U_{\lambda}(\eta, k)=i,
$$

is valid for both cases of real and imaginary $\lambda$, and that its flat space limit is simple,

$$
U_{\lambda}(\eta, k) \stackrel{H_{0} \rightarrow 0}{\longrightarrow} \frac{e^{-i k \eta}}{\sqrt{2 k}} .
$$

For spatially homogeneous states the complex integration constants $A$ and $B$ depend on the magnitude of the momentum $k=\|\vec{k}\|$ only, and furthermore the state normalization condition (given by the Klein-Gordon product) imposes, $|A|^{2}-|B|^{2}=1$. The choice $A=1, B=0(\forall \vec{k})$ corresponds to the CTBD vacuum state [97, 98]. In general FLRW space-times formal solutions for the scalar mode functions were given in [119]. However, explicit solutions are available only in a handful of cases, power-law inflation we consider here being one.

\section{B Rescaled propagator function $\mathcal{F}_{\nu}$}

In (5.5) we have introduced the rescaled propagator function,

$$
\mathcal{F}_{\lambda}(y)=\frac{\left[(1-\epsilon) H_{0}\right]^{D-2}}{(4 \pi)^{D / 2}} \frac{\Gamma\left(\frac{D-1}{2}+\lambda\right) \Gamma\left(\frac{D-1}{2}-\lambda\right)}{\Gamma\left(\frac{D}{2}\right)}{ }_{2} F_{1}\left(\left\{\frac{D-1}{2}+\lambda, \frac{D-1}{2}-\lambda\right\},\left\{\frac{D}{2}\right\}, 1-\frac{y}{4}\right) .
$$


where ${ }_{2} F_{1}$ is the Gauss' hypergeometric function. It appears in the solution for the twopoint functions of a massless non-minimally coupled scalar in power-law inflation (5.4) (see refs. $[8,14])$,

$$
i\left[{ }^{A} \Delta^{B}\right]_{\nu_{\xi}}\left(x ; x^{\prime}\right)=\left(a a^{\prime}\right)^{-\frac{(D-2) \epsilon}{2}} \times \mathcal{F}_{\nu_{\xi}}\left(y_{A B}\right),
$$

where $A, B$ are the Schwinger-Keldysh polarities of the distance function defined in (2.13), and parameter $\nu_{\xi}$ is related to the non-minimal coupling of the scalar as,

$$
\nu_{\xi}^{2}=\left(\frac{D-1-\epsilon}{2(1-\epsilon)}\right)^{2}-\frac{(D-1)(D-2 \epsilon)}{(1-\epsilon)^{2}} \xi
$$

The rescaled propagator function satisfies the hypergeometric equation in disguise,

$$
\left[\left(4 y-y^{2}\right) \frac{\partial^{2}}{\partial y^{2}}+D(2-y) \frac{\partial}{\partial y}+\lambda^{2}-\left(\frac{D-1}{2}\right)^{2}\right] \mathcal{F}_{\lambda}(y)=0 .
$$

At multiple instances we need the power series expansion of the rescaled propagator function around $y=0$. To derive it we first use the identity (15.8.4) from [120, 121] (or identity (9.131.2) from [104]) for the transformation of argument of the hypergeometric function, and write (B.1) as

$$
\begin{aligned}
\mathcal{F}_{\lambda}(y)= & \frac{\left[(1-\epsilon) H_{0}\right]^{D-2}}{(4 \pi)^{D / 2}} \Gamma\left(\frac{D-2}{2}\right)\left\{\left(\frac{y}{4}\right)^{-\frac{D-2}{2}} \times{ }_{2} F_{1}\left(\left\{\frac{1}{2}+\lambda, \frac{1}{2}-\lambda\right\},\left\{\frac{4-D}{2}\right\}, \frac{y}{4}\right)\right. \\
& \left.+\frac{\Gamma\left(\frac{2-D}{2}\right) \Gamma\left(\frac{D-1}{2}+\lambda\right) \Gamma\left(\frac{D-1}{2}-\lambda\right)}{\Gamma\left(\frac{D-2}{2}\right) \Gamma\left(\frac{1}{2}+\lambda\right) \Gamma\left(\frac{1}{2}-\lambda\right)} \times{ }_{2} F_{1}\left(\left\{\frac{D-1}{2}+\lambda, \frac{D-1}{2}-\lambda\right\},\left\{\frac{D}{2}\right\}, \frac{y}{4}\right)\right\},
\end{aligned}
$$

which is then straightforward to expand in the desired power series,

$$
\begin{aligned}
\mathcal{F}_{\lambda}(y)= & {\left[(1-\epsilon) H_{0}\right]^{D-2} \times \frac{\Gamma\left(\frac{D-2}{2}\right)}{(4 \pi)^{D / 2}} \times\left\{\left(\frac{y}{4}\right)^{-\frac{D-2}{2}}+\frac{\Gamma\left(\frac{4-D}{2}\right)}{\Gamma\left(\frac{1}{2}+\lambda\right) \Gamma\left(\frac{1}{2}-\lambda\right)} \times \sum_{n=0}^{\infty}\right.} \\
& \left.\times\left[\frac{\Gamma\left(\frac{3}{2}+\lambda+n\right) \Gamma\left(\frac{3}{2}-\lambda+n\right)}{\Gamma\left(\frac{6-D}{2}+n\right) \Gamma(n+2)}\left(\frac{y}{4}\right)^{n-\frac{D-4}{2}}-\frac{\Gamma\left(\frac{D-1}{2}+\lambda+n\right) \Gamma\left(\frac{D-1}{2}-\lambda+n\right)}{\Gamma\left(\frac{D}{2}+n\right) \Gamma(n+1)}\left(\frac{y}{4}\right)^{n}\right]\right\} .
\end{aligned}
$$

We also need an asymptotic expansion for large $y$ in section 8. To obtain it we first apply the identity 9.132 .1 from [104] to transform the argument of the hypergeometric function,

$$
\begin{aligned}
\mathcal{F}(y)= & \frac{\left[(1-\epsilon) H_{0}\right]^{D-2}}{(4 \pi)^{D / 2}} \\
& \times\left\{\frac{\Gamma(2 \lambda) \Gamma\left(\frac{D-1}{2}-\lambda\right)}{\Gamma\left(\frac{1}{2}+\lambda\right)}\left(\frac{y}{4}\right)^{-\frac{D-1}{2}+\lambda}{ }_{2} F_{1}\left(\left\{\frac{D-1}{2}-\lambda, \frac{1}{2}-\lambda\right\},\{1-2 \lambda\}, \frac{4}{y}\right)\right. \\
& \left.\quad+\frac{\Gamma(-2 \lambda) \Gamma\left(\frac{D-1}{2}+\lambda\right)}{\Gamma\left(\frac{1}{2}+\lambda\right)}\left(\frac{y}{4}\right)^{-\frac{D-1}{2}-\lambda}{ }_{2} F_{1}\left(\left\{\frac{D-1}{2}+\lambda, \frac{1}{2}+\lambda\right\},\{1+2 \lambda\}, \frac{4}{y}\right)\right\},
\end{aligned}
$$


after which the expansion for large $y$ is straightforward,

$$
\begin{array}{r}
\mathcal{F}_{\lambda}(y) \stackrel{y \rightarrow \infty}{\sim} \frac{\left[(1-\epsilon) H_{0}\right]^{D-2}}{(4 \pi)^{D / 2}}\left\{\frac{\Gamma(2 \lambda) \Gamma\left(\frac{D-1}{2}-\lambda\right)}{\Gamma\left(\frac{1}{2}+\lambda\right)}\left(\frac{y}{4}\right)^{-\frac{D-1}{2}+\lambda}\left[1+\frac{(D-1-2 \lambda)}{y}+\ldots\right]\right. \\
\left.+\frac{\Gamma(-2 \lambda) \Gamma\left(\frac{D-1}{2}+\lambda\right)}{\Gamma\left(\frac{1}{2}-\lambda\right)}\left(\frac{y}{4}\right)^{-\frac{D-1}{2}-\lambda}\left[1+\frac{(D-1+2 \lambda)}{y}+\ldots\right]\right\}
\end{array}
$$

\section{Useful identity for $\mathcal{F}_{\nu}$}

In this appendix we derive a very useful identity which allows us to eliminate the inverse Laplace operator from the expression for the vector two-point function in section 7.3. The variables used to express the two-point function introduced in (2.11) and (7.39) are,

$$
y=(1-\epsilon)^{2} \mathcal{H} \mathcal{H}^{\prime}\left[\|\Delta \vec{x}\|^{2}-\left(\eta-\eta^{\prime}\right)^{2}\right], \quad u=(1-\epsilon) \ln \left(a a^{\prime}\right), \quad v=(1-\epsilon) \ln \left(a / a^{\prime}\right),
$$

where $\mathcal{H}=H_{0} a^{1-\epsilon}=H_{0} e^{(u+v) / 2}, \mathcal{H}^{\prime}=H_{0} a^{1-\epsilon}=H_{0} e^{(u-v) / 2}$, and $(1-\epsilon)^{2} \mathcal{H} \mathcal{H}^{\prime}\left(\eta-\eta^{\prime}\right)^{2}=$ $2[\operatorname{ch}(v)-1]$. In this appendix all expressions are valid for $y_{-+}\left(x ; x^{\prime}\right)$ only, but we suppress the explicit Schwinger-Keldish polarities. At multiple instances we make use of the first and second space or time derivatives of $y\left(x ; x^{\prime}\right)$,

$$
\begin{aligned}
\left(\partial_{0} y\right) & =(1-\epsilon)\left[\mathcal{H} y-2\left(\mathcal{H}-\mathcal{H}^{\prime}\right)\right]=(1-\epsilon) H_{0} e^{\frac{u+v}{2}}\left[2 e^{-v}-(2-y)\right], \\
\left(\partial_{0}^{\prime} y\right) & =(1-\epsilon)\left[\mathcal{H}^{\prime} y+2\left(\mathcal{H}-\mathcal{H}^{\prime}\right)\right]=(1-\epsilon) H_{0} e^{\frac{u-v}{2}}\left[2 e^{v}-(2-y)\right], \\
\left(\partial_{i} y\right) & =-\left(\partial_{i}^{\prime} y\right)=2(1-\epsilon)^{2} \mathcal{H} \mathcal{H}^{\prime} \Delta x_{i}=2(1-\epsilon)^{2} H_{0}^{2} e^{u} \Delta x_{i} \\
\left(\partial_{0} \partial_{0}^{\prime} y\right) & =(1-\epsilon)^{2}\left[2 \mathcal{H}^{2}+2\left(\mathcal{H}^{\prime}\right)^{2}-\mathcal{H H}^{\prime}(2-y)\right]=(1-\epsilon)^{2} H_{0}^{2} e^{u}[4 \operatorname{ch}(v)-(2-y)], \\
\left(\partial_{0} \partial_{i} y\right) & =-\left(\partial_{0} \partial_{i}^{\prime} y\right)=(1-\epsilon) \mathcal{H}\left(\partial_{i} y\right)=(1-\epsilon) H_{0} e^{\frac{u+v}{2}}\left(\partial_{i} y\right), \\
\left(\partial_{0}^{\prime} \partial_{i} y\right) & =-\left(\partial_{0}^{\prime} \partial_{i}^{\prime} y\right)=(1-\epsilon) \mathcal{H}^{\prime}\left(\partial_{i} y\right)=(1-\epsilon) H_{0} e^{\frac{u-v}{2}}\left(\partial_{i} y\right), \\
\left(\partial_{i} \partial_{j} y\right) & =-\left(\partial_{i} \partial_{j}^{\prime} y\right)=-\left(\partial_{i}^{\prime} \partial_{j} y\right)=\left(\partial_{i}^{\prime} \partial_{j}^{\prime} y\right)=2 \delta_{i j}(1-\epsilon)^{2} \mathcal{H} \mathcal{H}^{\prime}=2 \delta_{i j}(1-\epsilon)^{2} H_{0}^{2} e^{u} .
\end{aligned}
$$

Using the derivatives above we can derive a simple derivative identity for the functions dependent on $y$ only,

$$
\frac{1}{2} \nabla^{2} I[f](y)=(1-\epsilon)\left[\mathcal{H}^{\prime} \partial_{0}+\mathcal{H} \partial_{0}^{\prime}+(D-1)(1-\epsilon) \mathcal{H} \mathcal{H}^{\prime}\right] f(y),
$$

where $I[f](y) \equiv \int^{y} d y^{\prime} f\left(y^{\prime}\right)$ denotes the primitive function of $f(y)$. Proving it involves showing that

$$
\left[\mathcal{H}^{\prime} \partial_{0}+\mathcal{H} \partial_{0}^{\prime}\right] y=\frac{1}{2(1-\epsilon)}(\vec{\nabla} y) \cdot(\vec{\nabla} y)
$$

by making use of (C.5), (C.2), and (C.3), and then using this to compare the two sides of equality (C.9). Using the simple identity (C.9), and the differential equation (B.4) satisfied by $\mathcal{F}_{\lambda}(y)$ we can derive a more involved identity,

$$
\begin{aligned}
\partial_{0} \partial_{0}^{\prime}\left[\left(a a^{\prime}\right)^{\rho} \mathcal{F}_{\lambda}(y)\right]=\left(a a^{\prime}\right)^{\rho} \times\{ & -\frac{1}{2} \nabla^{2} I\left[(2-y) \frac{\partial \mathcal{F}_{\lambda}}{\partial y}-\frac{\rho}{(1-\epsilon)} \mathcal{F}_{\lambda}\right] \\
& \left.-(1-\epsilon)^{2} \mathcal{H} \mathcal{H}^{\prime}\left[\lambda^{2}-\left(\frac{D-1}{2}-\frac{\rho}{1-\epsilon}\right)^{2}\right] \mathcal{F}_{\lambda}\right\},
\end{aligned}
$$


which we use in section 7.3. This identity can be proven in several steps outlined below:

(i) Show the two relations,

$$
\begin{aligned}
\left(\partial_{0} y\right)\left(\partial_{0}^{\prime} y\right) & =-(1-\epsilon)(2-y)\left[\mathcal{H} \partial_{0}^{\prime}+\mathcal{H}^{\prime} \partial_{0}\right] y+(1-\epsilon)^{2} \mathcal{H} \mathcal{H}^{\prime}\left(4 y-y^{2}\right) \\
\left(\partial_{0} \partial_{0}^{\prime} y\right) & =(1-\epsilon)\left[\mathcal{H} \partial_{0}^{\prime}+\mathcal{H}^{\prime} \partial_{0}\right] y+(1-\epsilon)^{2} \mathcal{H} \mathcal{H}^{\prime}(2-y)
\end{aligned}
$$

that follow from the basic derivative relations (C.2)-(C.8).

(ii) Use the two relations (C.12) and (C.13) to write out two derivatives action on some function dependent only on the length function $y$,

$$
\begin{aligned}
\partial_{0} \partial_{0}^{\prime} f(y)= & -(1-\epsilon)(2-y) \frac{\partial^{2} f}{\partial y^{2}}\left[\mathcal{H} \partial_{0}^{\prime}+\mathcal{H}^{\prime} \partial_{0}\right] y+(1-\epsilon) \frac{\partial f}{\partial y}\left[\mathcal{H} \partial_{0}^{\prime}+\mathcal{H}^{\prime} \partial_{0}\right] y \\
& +(1-\epsilon)^{2} \mathcal{H} \mathcal{H}^{\prime}\left(4 y-y^{2}\right) \frac{\partial^{2} f}{\partial y^{2}}+(1-\epsilon)^{2} \mathcal{H} \mathcal{H}^{\prime}(2-y) \frac{\partial f}{\partial y}
\end{aligned}
$$

Recognizing that $[\partial f / \partial y]\left(\partial_{0} y\right)=\partial_{0} f$ and applying the Leibnitz rule write the above expression as,

$$
\partial_{0} \partial_{0}^{\prime} f(y)=-(1-\epsilon)\left[\mathcal{H} \partial_{0}^{\prime}+\mathcal{H}^{\prime} \partial_{0}\right]\left[(2-y) \frac{\partial f}{\partial y}\right]+(1-\epsilon)^{2} \mathcal{H} \mathcal{H}^{\prime}\left[\left(4 y-y^{2}\right) \frac{\partial^{2} f}{\partial y^{2}}+(2-y) \frac{\partial f}{\partial y}\right] .
$$

(iii) Specialize to $f(y)=\mathcal{F}_{\lambda}(y)$ given in (B.1), and use the differential equation (B.4) to get rid of the second derivative in the last bracket of (C.15),

$$
\begin{aligned}
\partial_{0} \partial_{0}^{\prime} \mathcal{F}_{\lambda}(y)= & -(1-\epsilon)\left[\mathcal{H} \partial_{0}^{\prime}+\mathcal{H}^{\prime} \partial_{0}+(D-1)(1-\epsilon) \mathcal{H} \mathcal{H}^{\prime}\right]\left[(2-y) \frac{\partial \mathcal{F}_{\lambda}}{\partial y}\right] \\
& -(1-\epsilon)^{2} \mathcal{H} \mathcal{H}^{\prime}\left[\lambda^{2}-\left(\frac{D-1}{2}\right)^{2}\right] \mathcal{F}_{\lambda} .
\end{aligned}
$$

(iv) Apply the expression from the previous step to the left hand side of (C.11),

$$
\begin{array}{r}
\partial_{0} \partial_{0}^{\prime}\left[\left(a a^{\prime}\right)^{\rho} \mathcal{F}_{\lambda}(y)\right]=\left(a a^{\prime}\right)^{\rho} \times\left\{-(1-\epsilon)^{2} \mathcal{H} \mathcal{H}^{\prime}\left[\lambda^{2}-\left(\frac{D-1}{2}-\frac{\rho}{1-\epsilon}\right)^{2}\right] \mathcal{F}_{\lambda} \quad(\mathrm{C}\right. \\
\left.-(1-\epsilon)\left[\mathcal{H} \partial_{0}^{\prime}+\mathcal{H}^{\prime} \partial_{0}+(D-1)(1-\epsilon) \mathcal{H} \mathcal{H}^{\prime}\right]\left[(2-y) \frac{\partial \mathcal{F}_{\lambda}}{\partial y}-\frac{\rho}{(1-\epsilon)} \mathcal{F}_{\lambda}\right]\right\} .
\end{array}
$$

(v) Apply the simple identity (C.9) to the right hand side of (C.17), after which the identity (C.11) follows.

\section{Various derivative identities}

The covariantized solutions for the vector two-point functions given in (7.40), (7.55), with the scalar structure functions given in (7.47)-(7.50) have to respect properties dictated by canonical quantization. Namely, they have to satisfy transversality relations (7.13)-(7.15), 
and the equations of motion (7.16)-(7.18) Demonstrating that these hold involves taking multiple derivatives of the distance function $y_{A B}=(1-\epsilon)^{2} \mathcal{H} \mathcal{H}^{\prime} \Delta x_{A B}^{2}$, with either the $(-+)$ or the $(++) i \varepsilon$-prescription,

$$
\Delta x_{-+}^{2}=\left\|\vec{x}-\vec{x}^{\prime}\right\|^{2}-\left(\eta-\eta^{\prime}-i \varepsilon\right)^{2}, \quad \Delta x_{++}^{2}=\left\|\vec{x}-\vec{x}^{\prime}\right\|^{2}-\left(\left|\eta-\eta^{\prime}\right|-i \varepsilon\right)^{2} .
$$

In this appendix we collect the necessary derivative identities.

When taking derivatives of the $y_{-+}$appearing in the Wightman function, in practice we may just neglect the $i \varepsilon$-prescription, and restore it to each $y$ at the end. However, when the $i \varepsilon$-prescription corresponds to the Feynman or Dyson prescription, taking derivatives is particularly tricky, since local terms are generated. It is two or more time derivatives acting on $y_{++}$that generate $\delta$-functions in time,

$$
\begin{aligned}
\left(\partial_{0} \partial_{0} y_{++}\right) & =\left(\partial_{0} \partial_{0} y\right)+4(1-\epsilon)^{2} \mathcal{H}^{2} \times i \varepsilon \delta\left(\eta-\eta^{\prime}\right), \\
\left(\partial_{0} \partial_{0}^{\prime} y_{++}\right) & =\left(\partial_{0} \partial_{0}^{\prime} y\right)-4(1-\epsilon)^{2} \mathcal{H}^{2} \times i \varepsilon \delta\left(\eta-\eta^{\prime}\right), \\
\left(\partial_{0} \partial_{0} \partial_{0}^{\prime} y_{++}\right) & =\left(\partial_{0} \partial_{0} \partial_{0}^{\prime} y\right)-4(1-\epsilon)^{2} \mathcal{H}^{2}\left[\partial_{0}+2(1-\epsilon) \mathcal{H}\right] i \varepsilon \delta\left(\eta-\eta^{\prime}\right) .
\end{aligned}
$$

Whenever an ordinary spatial derivative acts on $y_{++}$no $\delta$-function in time can be generated from it. Identities with different combinations of primed and unprimed derivatives are derived from the above ones by simply switching the time arguments. This will be the case for all the expressions in this appendix, including spatial derivatives.

The tensor structures that harbour local terms in time multiply the scalar structure functions (7.47)-(7.50), which can be seen as power series in $y_{++}$, and this can result in a space-time local term that survives the limit $\varepsilon \rightarrow 0$. The space-time local terms are identified by making use of a representation for the covariant delta-function,

$$
i \varepsilon \delta\left(\eta-\eta^{\prime}\right) \times\left(\frac{y_{++}}{4}\right)^{-\frac{D}{2}}=[(1-\epsilon) H]^{-D} \frac{(4 \pi)^{D / 2}}{\Gamma\left(\frac{D}{2}\right)} \times \frac{i \delta^{D}\left(x-x^{\prime}\right)}{\sqrt{-g}} .
$$

Different combinations of derivatives acting on the identity above generate further identities,

$$
\begin{aligned}
i \varepsilon \delta\left(\eta-\eta^{\prime}\right)\left(\partial_{i} y\right)\left(\frac{y_{++}}{4}\right)^{-\frac{D+2}{2}}= & {[(1-\epsilon) H]^{-D} \frac{(4 \pi)^{D / 2}}{\Gamma\left(\frac{D}{2}\right)} \times\left(-\frac{8}{D}\right) \partial_{i} \frac{i \delta^{D}\left(x-x^{\prime}\right)}{\sqrt{-g}} } \\
i \varepsilon \delta\left(\eta-\eta^{\prime}\right) \partial_{i}\left[\left(\partial_{j} y\right)\left(\frac{y_{++}}{4}\right)^{-\frac{D+2}{2}}\right]= & {[(1-\epsilon) H]^{-D} \frac{(4 \pi)^{D / 2}}{\Gamma\left(\frac{D}{2}\right)} \times\left(-\frac{8}{D}\right) \partial_{i} \partial_{j} \frac{i \delta^{D}\left(x-x^{\prime}\right)}{\sqrt{-g}}, } \\
i \varepsilon \delta\left(\eta-\eta^{\prime}\right)\left(\frac{y_{++}}{4}\right)^{-\frac{D+2}{2}}= & {[(1-\epsilon) H]^{-D-2} \frac{(4 \pi)^{D / 2}}{\Gamma\left(\frac{D}{2}\right)} } \\
& \times\left(\frac{4}{3 D}\right) a^{-2} \nabla^{2} \frac{i \delta^{D}\left(x-x^{\prime}\right)}{\sqrt{-g}} \\
i \varepsilon \delta\left(\eta-\eta^{\prime}\right)\left(\partial_{0} y_{++}\right)\left(\frac{y_{++}}{4}\right)^{-\frac{D}{2}}= & {[(1-\epsilon) H]^{-D} \frac{(4 \pi)^{D / 2}}{\Gamma\left(\frac{D}{2}\right)} \times 4(1-\epsilon) \mathcal{H} \frac{i \delta^{D}\left(x-x^{\prime}\right)}{\sqrt{-g}} }
\end{aligned}
$$




\begin{tabular}{|lr|}
\hline$g^{\mu \nu}\left(\partial_{\mu} y\right)\left(\partial_{\nu} y\right)$ & $(1-\epsilon)^{2} H^{2}\left(4 y-y^{2}\right)$ \\
\hline$g^{\prime \rho \sigma}\left(\partial_{\rho}^{\prime} y\right)\left(\partial_{\sigma}^{\prime} y\right)$ & $(1-\epsilon)^{2} H^{\prime 2}\left(4 y-y^{2}\right)$ \\
\hline$g^{\mu \nu}\left(\partial_{\mu} y\right)\left(\partial_{\nu} u\right)$ & $(1-\epsilon)^{2} H^{2}\left(2-y-2 e^{-v}\right)$ \\
\hline$g^{\prime \rho \sigma}\left(\partial_{\rho}^{\prime} y\right)\left(\partial_{\sigma}^{\prime} u\right)$ & $(1-\epsilon)^{2} H^{\prime 2}\left(2-y-2 e^{v}\right)$ \\
\hline$g^{\mu \nu}\left(\partial_{\mu} u\right)\left(\partial_{\nu} u\right)$ & $-(1-\epsilon)^{2} H^{2}$ \\
\hline$g^{\prime \rho \sigma}\left(\partial_{\rho}^{\prime} u\right)\left(\partial_{\sigma}^{\prime} u\right)$ & $-(1-\epsilon)^{2} H^{\prime 2}$ \\
\hline$g^{\mu \nu}\left(\partial_{\mu} y\right)\left(\partial_{\nu} \partial_{\rho}^{\prime} y\right)$ & $(1-\epsilon)^{2} H^{2}(2-y)\left(\partial_{\rho}^{\prime} y\right)$ \\
\hline$g^{\prime \rho \sigma}\left(\partial_{\mu} \partial_{\rho}^{\prime} y\right)\left(\partial_{\sigma}^{\prime} y\right)$ & $-(1-\epsilon)^{2} H^{\prime 2}(2-y)\left(\partial_{\mu} y\right)$ \\
\hline$g^{\mu \nu}\left(\partial_{\mu} u\right)\left(\partial_{\nu} \partial_{\rho}^{\prime} y\right)$ & $-(1-\epsilon)^{2} H^{\prime 2}\left[\left(\partial_{\mu}^{\prime} y\right)+2 e^{-v}\left(\partial_{\rho}^{\prime} u\right)\right]$ \\
\hline$g^{\prime \rho \sigma}\left(\partial_{\mu} \partial_{\rho}^{\prime} y\right)\left(\partial_{\sigma}^{\prime} u\right)$ & $(1-\epsilon)^{2} H^{2}\left[4(1-\epsilon)^{2} H^{\prime 2} g_{\rho \sigma}^{\prime}-\left(\partial_{\rho}^{\prime} y\right)\left(\partial_{\sigma}^{\prime} y\right)\right]$ \\
\hline$g^{\mu \nu}\left(\partial_{\mu} \partial_{\rho}^{\prime} y\right)\left(\partial_{\nu} \partial_{\sigma}^{\prime} y\right)$ & $(1-\epsilon)^{2} H^{\prime 2}\left[4(1-\epsilon)^{2} H^{2} g_{\mu \nu}-\left(\partial_{\mu} y\right)\left(\partial_{\nu} y\right)\right]$ \\
\hline$g^{\prime \rho \sigma}\left(\partial_{\mu} \partial_{\rho}^{\prime} y\right)\left(\partial_{\nu} \partial_{\sigma}^{\prime} y\right)$ & \\
\hline
\end{tabular}

Table 2. Contractions of tensor structures. The expressions in the table are strictly speaking valid for distance functions $y$ without the $i \varepsilon$-prescription. In case the distance functions have $i \varepsilon$ prescriptions $y_{-+}$or $y_{++}$the expressions above are valid up to an innocuous term linear in $\varepsilon$, which can be neglected in all the expressions where contractions are used in this paper. In the case of double derivatives and the $(++) i \varepsilon$-prescription, the modified derivative $\overline{\left(\partial_{\mu} \partial_{\rho}^{\prime} y_{++}\right)}$defined in eq. (7.53) is assumed.

$$
\begin{aligned}
i \varepsilon \partial_{0} \delta\left(\eta-\eta^{\prime}\right)\left(\frac{y_{++}}{4}\right)^{-\frac{D}{2}}= & {[(1-\epsilon) H]^{-D} \frac{(4 \pi)^{D / 2}}{\Gamma\left(\frac{D}{2}\right)} } \\
& \times\left[\partial_{0}+\frac{1}{2} D(1+\epsilon) \mathcal{H}\right] \frac{i \delta^{D}\left(x-x^{\prime}\right)}{\sqrt{-g}} \\
i \varepsilon \partial_{0} \delta\left(\eta-\eta^{\prime}\right)\left(\partial_{i} y\right)\left(\frac{y_{++}}{4}\right)^{-\frac{D+2}{2}}= & {[(1-\epsilon) H]^{-D} \frac{(4 \pi)^{D / 2}}{\Gamma\left(\frac{D}{2}\right)} } \\
& \times\left(-\frac{8}{D}\right)\left[\partial_{0}+\frac{1}{2} D(1+\epsilon) \mathcal{H}\right] \partial_{i} \frac{i \delta^{D}\left(x-x^{\prime}\right)}{\sqrt{-g}} .
\end{aligned}
$$

It is useful to be able to reduce the derivative order, which is accomplished using the following two expressions,

$$
\begin{aligned}
& \left(\nabla_{\mu} \nabla_{\nu} y\right)=g_{\mu \nu}(1-\epsilon) H^{2}\left(2-y-2 \epsilon e^{-v}\right)-\frac{\epsilon}{(1-\epsilon)}\left[\left(\partial_{\mu} y\right)\left(\partial_{\nu} u\right)+\left(\partial_{\mu} u\right)\left(\partial_{\nu} y\right)\right], \\
& \left(\nabla_{\mu} \nabla_{\nu} u\right)=-g_{\mu \nu}(1-\epsilon) H^{2}-\left(\frac{1+\epsilon}{1-\epsilon}\right)\left(\partial_{\mu} u\right)\left(\partial_{\nu} u\right),
\end{aligned}
$$

where for higher derivatives we must keep in mind that the Hubble rate is not a constant, but $H^{2}=H_{0}^{2} e^{-\frac{(u+v) \epsilon}{(1-\epsilon)}}$. Furthermore, contractions of different tensor structures appear in the transversality relations, and equations of motion, and it is useful to have contraction identities given in table 2. The expressions below are ready made to be used in checking that the Wightman function and the Feynman propagator solutions from section 7.4 satisfy 
the correct transversality relations, and the correct equations of motion from section 7.1,

$$
\begin{aligned}
& \left(\nabla_{\mu} \nabla_{\nu} y_{++}\right)=g_{\mu \nu}(1-\epsilon) H^{2}\left[2-y_{++}-2 \epsilon e^{-v}\right] \\
& -\frac{2 \epsilon}{(1-\epsilon)}\left(\nabla_{(\mu} u\right)\left(\nabla_{\nu)} y_{++}\right)+\left(\nabla_{\mu} u\right)\left(\nabla_{\nu} u\right) \times 4 i \varepsilon \delta\left(\eta-\eta^{\prime}\right), \\
& \left(\nabla_{\mu} \nabla_{\rho}^{\prime} y_{++}\right)=\left(\overline{\nabla_{\mu} \nabla_{\rho}^{\prime} y_{++}}\right)-\left(\nabla_{\mu} u\right)\left(\nabla_{\rho}^{\prime} u\right) \times 4 i \varepsilon \delta\left(\eta-\eta^{\prime}\right), \\
& \left(\nabla_{\mu} \nabla_{\nu} \nabla_{\rho}^{\prime} y_{++}\right)=-(1-\epsilon) H^{2} g_{\mu \nu}\left(\nabla_{\rho}^{\prime} y_{++}\right) \\
& -2(1-\epsilon) H^{2} g_{\mu \nu}\left(\nabla_{\rho}^{\prime} u\right)\left[\epsilon e^{-v}-2 i \varepsilon \delta\left(\eta-\eta^{\prime}\right)\right]-\frac{2 \epsilon}{(1-\epsilon)}\left(\nabla_{(\mu} u\right)\left(\overline{\left.\nabla_{\nu}\right)} \nabla_{\rho}^{\prime} y_{++}\right) \\
& +\left(\nabla_{\mu} u\right)\left(\nabla_{\nu} u\right)\left(\nabla_{\rho}^{\prime} u\right)\left[\frac{8 \epsilon}{(1-\epsilon)} i \varepsilon \delta\left(\eta-\eta^{\prime}\right)+\frac{4}{(1-\epsilon) \mathcal{H}^{\prime}} \partial_{0}^{\prime} i \varepsilon \delta\left(\eta-\eta^{\prime}\right)\right], \\
& \left(\nabla_{\mu} \overline{\nabla_{\nu} \nabla_{\rho}^{\prime} y_{++}}\right)=-(1-\epsilon) H^{2} g_{\mu \nu}\left(\nabla_{\rho}^{\prime} y_{++}\right) \\
& -2 \epsilon(1-\epsilon) H^{2} e^{-v} g_{\mu \nu}\left(\nabla_{\rho}^{\prime} u\right)-\frac{2 \epsilon}{(1-\epsilon)}\left(\nabla_{(\mu} u\right)\left(\overline{\left.\nabla_{\nu}\right)} \nabla_{\rho}^{\prime} y_{++}\right), \\
& \left(\nabla_{\alpha} \nabla_{\mu} \overline{\nabla_{\nu} \nabla_{\rho}^{\prime} y_{++}}\right)=-(1-\epsilon) H^{2} g_{\mu \nu}\left(\overline{\nabla_{\alpha} \nabla_{\rho}^{\prime} y_{++}}\right) \\
& -2(1-\epsilon) g_{\mu \nu}\left(\nabla_{\rho}^{\prime} u\right) \nabla_{\alpha}\left[H^{2} \epsilon e^{-v}\right]-\frac{2 \epsilon}{(1-\epsilon)}\left(\nabla_{\alpha} \nabla_{(\mu} u\right)\left(\overline{\nabla_{\nu)} \nabla_{\rho}^{\prime} y_{++}}\right) \\
& -\frac{2 \epsilon}{(1-\epsilon)}\left(\nabla_{(\mu} u\right)\left(\nabla_{\alpha} \overline{\nabla_{\nu)} \nabla_{\rho}^{\prime} y_{++}}\right) \text {. }
\end{aligned}
$$

Open Access. This article is distributed under the terms of the Creative Commons Attribution License (CC-BY 4.0), which permits any use, distribution and reproduction in any medium, provided the original author(s) and source are credited.

\section{References}

[1] L. Parker, Particle creation in expanding universes, Phys. Rev. Lett. 21 (1968) 562 [INSPIRE].

[2] L. Parker, Quantized fields and particle creation in expanding universes. 1, Phys. Rev. 183 (1969) 1057 [inSPIRE].

[3] L. Parker, Quantized fields and particle creation in expanding universes. 2, Phys. Rev. D 3 (1971) 346 [Erratum ibid. 3 (1971) 2546] [INSPIRE].

[4] R.P. Woodard, Perturbative quantum gravity comes of age, Int. J. Mod. Phys. D 23 (2014) 1430020 [arXiv:1407.4748] [InSPIRE].

[5] S. Weinberg, Quantum contributions to cosmological correlations, Phys. Rev. D 72 (2005) 043514 [hep-th/0506236] [INSPIRE].

[6] S. Weinberg, Quantum contributions to cosmological correlations. II. Can these corrections become large?, Phys. Rev. D 74 (2006) 023508 [hep-th/0605244] [INSPIRE].

[7] D. Glavan and G. Rigopoulos, One-loop E\&M correlators of SQED in power-law inflation, arXiv: 1909.11741 [INSPIRE].

[8] T.M. Janssen, S.P. Miao, T. Prokopec and R.P. Woodard, Infrared propagator corrections for constant deceleration, Class. Quant. Grav. 25 (2008) 245013 [arXiv:0808.2449] [INSPIRE].

[9] F. Lucchin and S. Matarrese, Power law inflation, Phys. Rev. D 32 (1985) 1316 [inSPIRE]. 
[10] D. La and P.J. Steinhardt, Extended inflationary cosmology, Phys. Rev. Lett. 62 (1989) 376 [Erratum ibid. 62 (1989) 1066] [INSPIRE].

[11] Planck collaboration, Planck 2018 results. I. Overview and the cosmological legacy of Planck, arXiv:1807.06205 [INSPIRE].

[12] L.R.W. Abramo and R.P. Woodard, One loop back reaction on power law inflation, Phys. Rev. D 60 (1999) 044011 [astro-ph/9811431] [INSPIRE].

[13] T.M. Janssen, S.P. Miao, T. Prokopec and R.P. Woodard, The Hubble effective potential, JCAP 05 (2009) 003 [arXiv:0904.1151] [InSPIRE].

[14] T. Janssen and T. Prokopec, The graviton one-loop effective action in cosmological space-times with constant deceleration, Annals Phys. 325 (2010) 948 [arXiv:0807.0447] [INSPIRE].

[15] M. Herranen, T. Markkanen and A. Tranberg, Quantum corrections to scalar field dynamics in a slow-roll space-time, JHEP 05 (2014) 026 [arXiv: 1311.5532] [INSPIRE].

[16] T. Prokopec, Late time solution for interacting scalar in accelerating spaces, JCAP 11 (2015) 016 [arXiv: 1508.07874] [INSPIRE].

[17] G. Cho, C.H. Kim and H. Kitamoto, Stochastic dynamics of infrared fluctuations in accelerating universe, in $2^{\text {nd }}$ LeCosPA symposium: everything about gravity, celebrating the centenary of Einstein's general relativity, World Scientific, Singapore (2017), pg. 162 [arXiv: 1508.07877] [INSPIRE].

[18] T. Markkanen, Light scalars on cosmological backgrounds, JHEP 01 (2018) 116 [arXiv:1711.07502] [INSPIRE].

[19] A. Kyriazis, S.P. Miao, N.C. Tsamis and R.P. Woodard, The inflaton effective potential for general $\epsilon$, arXiv: 1908.03814 [INSPIRE].

[20] A. Dolgov, Breaking of conformal invariance and electromagnetic field generation in the universe, Phys. Rev. D 48 (1993) 2499 [hep-ph/9301280] [INSPIRE].

[21] I.T. Drummond and S.J. Hathrell, QED vacuum polarization in a background gravitational field and its effect on the velocity of photons, Phys. Rev. D 22 (1980) 343 [InSPIRE].

[22] T. Prokopec, Cosmological magnetic fields from photon coupling to fermions and bosons in inflation, astro-ph/0106247 [INSPIRE].

[23] A. Benevides, A. Dabholkar and T. Kobayashi, To B or not to B: primordial magnetic fields from Weyl anomaly, JHEP 11 (2018) 039 [arXiv:1808.08237] [INSPIRE].

[24] T. Prokopec, O. Tornkvist and R.P. Woodard, Photon mass from inflation, Phys. Rev. Lett. 89 (2002) 101301 [astro-ph/0205331] [inSPIRE].

[25] T. Prokopec, O. Tornkvist and R.P. Woodard, One loop vacuum polarization in a locally de Sitter background, Annals Phys. 303 (2003) 251 [gr-qc/0205130] [INSPIRE].

[26] T. Prokopec and E. Puchwein, Photon mass generation during inflation: de Sitter invariant case, JCAP 04 (2004) 007 [astro-ph/0312274] [INSPIRE].

[27] T. Prokopec, N.C. Tsamis and R.P. Woodard, Stochastic inflationary scalar electrodynamics, Annals Phys. 323 (2008) 1324 [arXiv:0707.0847] [INSPIRE].

[28] J.F. Koksma and T. Prokopec, Fermion propagator in cosmological spaces with constant deceleration, Class. Quant. Grav. 26 (2009) 125003 [arXiv:0901.4674] [INSPIRE]. 
[29] T. Prokopec and R.P. Woodard, Vacuum polarization and photon mass in inflation, Am. J. Phys. 72 (2004) 60 [astro-ph/0303358] [InSPIRE].

[30] T. Prokopec and R.P. Woodard, Dynamics of superhorizon photons during inflation with vacuum polarization, Annals Phys. 312 (2004) 1 [gr-qc/0310056] [INSPIRE].

[31] A.A. Starobinsky, Stochastic de Sitter (inflationary) stage in the early universe, Lect. Notes Phys. 246 (1986) 107 [INSPIRE].

[32] W.Z. Chua, Q. Ding, Y. Wang and S. Zhou, Imprints of Schwinger effect on primordial spectra, JHEP 04 (2019) 066 [arXiv:1810.09815] [INSPIRE].

[33] S. Lu, Y. Wang and Z.-Z. Xianyu, A cosmological Higgs collider, JHEP 02 (2020) 011 [arXiv: 1907.07390] [INSPIRE].

[34] X. Chen, Y. Wang and Z.-Z. Xianyu, Loop corrections to Standard Model fields in inflation, JHEP 08 (2016) 051 [arXiv:1604.07841] [INSPIRE].

[35] T. Prokopec and E. Puchwein, Nearly minimal magnetogenesis, Phys. Rev. D 70 (2004) 043004 [astro-ph/0403335] [INSPIRE].

[36] B. Ratra, Cosmological 'seed' magnetic field from inflation, Astrophys. J. Lett. 391 (1992) L1 [INSPIRE].

[37] T. Fujita and R. Durrer, Scale-invariant helical magnetic fields from inflation, JCAP 09 (2019) 008 [arXiv: 1904.11428] [INSPIRE].

[38] A. Brandenburg, R. Durrer, T. Kahniashvili, S. Mandal and W.W. Yin, Statistical properties of scale-invariant helical magnetic fields and applications to cosmology, JCAP 08 (2018) 034 [arXiv: 1804.01177] [INSPIRE].

[39] R. Durrer and A. Neronov, Cosmological magnetic fields: their generation, evolution and observation, Astron. Astrophys. Rev. 21 (2013) 62 [arXiv:1303.7121] [INSPIRE].

[40] A. Maleknejad, M.M. Sheikh-Jabbari and J. Soda, Gauge fields and inflation, Phys. Rept. 528 (2013) 161 [arXiv:1212. 2921] [INSPIRE].

[41] K. Subramanian, The origin, evolution and signatures of primordial magnetic fields, Rept. Prog. Phys. 79 (2016) 076901 [arXiv: 1504.02311] [INSPIRE].

[42] N. Bartolo et al., Science with the space-based interferometer LISA. IV: probing inflation with gravitational waves, JCAP 12 (2016) 026 [arXiv:1610.06481] [INSPIRE].

[43] C. Caprini et al., Science with the space-based interferometer eLISA. II: gravitational waves from cosmological phase transitions, JCAP 04 (2016) 001 [arXiv:1512.06239] [INSPIRE].

[44] C. Caprini and R. Durrer, Gravitational waves from stochastic relativistic sources: primordial turbulence and magnetic fields, Phys. Rev. D 74 (2006) 063521 [astro-ph/0603476] [INSPIRE].

[45] C. Caprini and R. Durrer, Gravitational wave production: a strong constraint on primordial magnetic fields, Phys. Rev. D 65 (2001) 023517 [astro-ph/0106244] [INSPIRE].

[46] C. Wetterich, Exact evolution equation for the effective potential, Phys. Lett. B 301 (1993) 90 [arXiv:1710.05815] [INSPIRE].

[47] G. Lazzari and T. Prokopec, Symmetry breaking in de Sitter: a stochastic effective theory approach, arXiv:1304.0404 [INSPIRE]. 
[48] J. Serreau, Renormalization group flow and symmetry restoration in de Sitter space, Phys. Lett. B $\mathbf{7 3 0}$ (2014) 271 [arXiv:1306.3846] [InSPIRE].

[49] M. Guilleux and J. Serreau, Quantum scalar fields in de Sitter space from the nonperturbative renormalization group, Phys. Rev. D 92 (2015) 084010 [arXiv: 1506.06183] [INSPIRE].

[50] F. Fabián González and T. Prokopec, Renormalization group approach to scalar quantum electrodynamics on de Sitter, arXiv:1611.07854 [INSPIRE].

[51] P.A.M. Dirac, Lectures on quantum mechanics, 1964 reprint, Dover Publications, Mineola, NY, U.S.A. (2011).

[52] D.M. Gitman and I.V. Tyutin, Quantization of fields with constraints, Springer, Berlin, Heidelberg, Germany (1990).

[53] H.J. Rothe and K.D. Rothe, Classical and quantum dynamics of constrained Hamiltonian systems, World Scientific, Singapore (2010).

[54] G. 't Hooft, Renormalizable Lagrangians for massive Yang-Mills fields, Nucl. Phys. B 35 (1971) 167 [inSPIRE].

[55] K. Fujikawa, B.W. Lee and A.I. Sanda, Generalized renormalizable gauge formulation of spontaneously broken gauge theories, Phys. Rev. D 6 (1972) 2923 [InSPIRE].

[56] T. Anderberg, Unitary gauge considered harmful, arXiv:0804.2284 [INSPIRE].

[57] S. Weinberg, General theory of broken local symmetries, Phys. Rev. D 7 (1973) 1068 [INSPIRE].

[58] S. Weinberg, Perturbative calculations of symmetry breaking, Phys. Rev. D 7 (1973) 2887 [INSPIRE].

[59] T. Appelquist, J. Carazzone, J. Goldman and H.R. Quinn, Renormalization and gauge independence in spontaneously broken gauge theories, Phys. Rev. D 8 (1973) 1747 [INSPIRE].

[60] C. Becchi, A. Rouet and R. Stora, The Abelian Higgs-Kibble model. Unitarity of the $S$ operator, Phys. Lett. B 52 (1974) 344 [InSPIRE].

[61] C. Becchi, A. Rouet and R. Stora, Renormalization of the Abelian Higgs-Kibble model, Commun. Math. Phys. 42 (1975) 127 [inSPIRE].

[62] B.W. Lee and J. Zinn-Justin, Spontaneously broken gauge symmetries part 1: preliminaries, Phys. Rev. D 5 (1972) 3121 [INSPIRE].

[63] B.W. Lee and J. Zinn-Justin, Spontaneously broken gauge symmetries part 2: perturbation theory and renormalization, Phys. Rev. D 5 (1972) 3137 [Erratum ibid. 8 (1973) 4654] [INSPIRE].

[64] B.W. Lee and J. Zinn-Justin, Spontaneously broken gauge symmetries part 3: equivalence, Phys. Rev. D 5 (1972) 3155 [INSPIRE].

[65] N.N. Bogoliubov and O.S. Parasiuk, On the multiplication of the causal function in the quantum theory of fields, Acta Math. 97 (1957) 227 [INSPIRE].

[66] K. Hepp, Proof of the Bogolyubov-Parasiuk theorem on renormalization, Commun. Math. Phys. 2 (1966) 301 [InSPIRE]. 
[67] W. Zimmermann, The power counting theorem for Minkowski metric, Commun. Math. Phys. 11 (1968) 1 [inSPIRE].

[68] S.Y. Lee, Higher-order corrections to leptonic processes and the renormalization of Weinberg's theory of weak interactions in the unitary gauge, Phys. Rev. D 6 (1972) 1701 [INSPIRE].

[69] G.B. Tupper, Unitarity and renormalizability - together at last, arXiv:1412.5959 [INSPIRE].

[70] W. Greiner and J. Reinhardt, Field quantization, Springer, Berlin, Heidelberg, Germany (1996).

[71] C.-N. Yang and D. Feldman, The $S$ matrix in the Heisenberg representation, Phys. Rev. 79 (1950) 972 [INSPIRE].

[72] J.S. Schwinger, Brownian motion of a quantum oscillator, J. Math. Phys. 2 (1961) 407 [INSPIRE].

[73] L.V. Keldysh, Diagram technique for nonequilibrium processes, Zh. Eksp. Teor. Fiz. 47 (1964) 1515 [Sov. Phys. JETP 20 (1965) 1018] [INSPIRE].

[74] S. Mooij and M. Postma, Goldstone bosons and a dynamical Higgs field, JCAP 09 (2011) 006 [arXiv:1104.4897] [InSPIRE].

[75] D.P. George, S. Mooij and M. Postma, Effective action for the Abelian Higgs model in FLRW, JCAP 11 (2012) 043 [arXiv:1207.6963] [INSPIRE].

[76] B. Allen and T. Jacobson, Vector two point functions in maximally symmetric spaces, Commun. Math. Phys. 103 (1986) 669 [InSPIRE].

[77] E.O. Kahya and R.P. Woodard, Charged scalar self-mass during inflation, Phys. Rev. D 72 (2005) 104001 [gr-qc/0508015] [INSPIRE].

[78] N.C. Tsamis and R.P. Woodard, A maximally symmetric vector propagator, J. Math. Phys. 48 (2007) 052306 [gr-qc/0608069] [InSPIRE].

[79] M.B. Fröb and A. Higuchi, Mode-sum construction of the two-point functions for the Stueckelberg vector fields in the Poincaré patch of de Sitter space, J. Math. Phys. 55 (2014) 062301 [arXiv:1305.3421] [INSPIRE].

[80] A. Belokogne, A. Folacci and J. Queva, Stueckelberg massive electromagnetism in de Sitter and anti-de Sitter spacetimes: two-point functions and renormalized stress-energy tensors, Phys. Rev. D 94 (2016) 105028 [arXiv:1610.00244] [InSPIRE].

[81] T. Prokopec, N.C. Tsamis and R.P. Woodard, Two loop scalar bilinears for inflationary SQED, Class. Quant. Grav. 24 (2007) 201 [gr-qc/0607094] [INSPIRE].

[82] T. Prokopec, N.C. Tsamis and R.P. Woodard, Two loop stress-energy tensor for inflationary scalar electrodynamics, Phys. Rev. D 78 (2008) 043523 [arXiv:0802.3673] [INSPIRE].

[83] D. Glavan, S.P. Miao, T. Prokopec and R.P. Woodard, Graviton loop corrections to vacuum polarization in de Sitter in a general covariant gauge, Class. Quant. Grav. 32 (2015) 195014 [arXiv:1504.00894] [INSPIRE].

[84] D. Glavan, S.P. Miao, T. Prokopec and R.P. Woodard, One loop graviton corrections to dynamical photons in de Sitter, Class. Quant. Grav. 34 (2017) 085002 [arXiv:1609.00386] [INSPIRE].

[85] P.A.M. Dirac, Generalized Hamiltonian dynamics, Can. J. Math. 2 (1950) 129 [InSPIRE]. 
[86] P.A.M. Dirac, The Hamiltonian form of field dynamics, Can. J. Math. 3 (1951) 1 [INSPIRE].

[87] J.L. Anderson and P.G. Bergmann, Constraints in covariant field theories, Phys. Rev. 83 (1951) 1018 [InSPIRE].

[88] Planck collaboration, Planck 2018 results. VI. Cosmological parameters, arXiv: 1807.06209 [INSPIRE].

[89] V.K. Onemli and R.P. Woodard, Superacceleration from massless, minimally coupled $\phi^{4}$, Class. Quant. Grav. 19 (2002) 4607 [gr-qc/0204065] [inSPIRE].

[90] N.C. Tsamis and R.P. Woodard, The structure of perturbative quantum gravity on a de Sitter background, Commun. Math. Phys. 162 (1994) 217 [InSPIRE].

[91] J. Berges, Introduction to nonequilibrium quantum field theory, AIP Conf. Proc. 739 (2004) 3 [hep-ph/0409233] [INSPIRE].

[92] D. Glavan and T. Prokopec, A pedestrian introduction to non-equilibrium QFT, http://www.staff.science.uu.nl/ proko101/LecturenotesNonEquilQFT.pdf.

[93] A. Golovnev, V. Mukhanov and V. Vanchurin, Vector inflation, JCAP 06 (2008) 009 [arXiv:0802.2068] [INSPIRE].

[94] A. Golovnev, V. Mukhanov and V. Vanchurin, Gravitational waves in vector inflation, JCAP 11 (2008) 018 [arXiv:0810.4304] [INSPIRE].

[95] K. Dimopoulos, Can a vector field be responsible for the curvature perturbation in the universe?, Phys. Rev. D $\mathbf{7 4}$ (2006) 083502 [hep-ph/0607229] [INSPIRE].

[96] K. Dimopoulos, M. Karciauskas, D.H. Lyth and Y. Rodriguez, Statistical anisotropy of the curvature perturbation from vector field perturbations, JCAP 05 (2009) 013 [arXiv:0809.1055] [INSPIRE].

[97] N.A. Chernikov and E.A. Tagirov, Quantum theory of scalar fields in de Sitter space-time, Ann. Inst. H. Poincaré Phys. Theor. A 9 (1968) 109 [inSPIRE].

[98] T.S. Bunch and P.C.W. Davies, Quantum field theory in de Sitter space: renormalization by point splitting, Proc. Roy. Soc. Lond. A A 360 (1978) 117 [inSPIRE].

[99] L.H. Ford and L. Parker, Infrared divergences in a class of Robertson-Walker universes, Phys. Rev. D 16 (1977) 245 [InSPIRE].

[100] T.M. Janssen and T. Prokopec, Regulating the infrared by mode matching: a massless scalar in expanding spaces with constant deceleration, Phys. Rev. D 83 (2011) 084035 [arXiv:0906.0666] [INSPIRE].

[101] A. Albrecht, P. Ferreira, M. Joyce and T. Prokopec, Inflation and squeezed quantum states, Phys. Rev. D 50 (1994) 4807 [astro-ph/9303001] [INSPIRE].

[102] I.I. Cotaescu, Polarized vector bosons on the de Sitter expanding universe, Gen. Rel. Grav. 42 (2010) 861 [arXiv:0901.3207] [InSPIRE].

[103] V. Mukhanov and S. Winitzki, Introduction to quantum effects in gravity, Cambridge University Press, Cambridge, U.K. (2007)

[104] I.S. Gradshteyn and I.M. Ryzhik, Table of integrals, series and products, seventh edition, A. Jeffrey and D. Zwillinger eds., Elsevier/Academic Press, Amsterdam, The Netherlands (2007). 
[105] G. Narain, Green's function of the vector fields on de-Sitter background, arXiv:1408.6193 [INSPIRE].

[106] T. Kobayashi and M.S. Sloth, Early cosmological evolution of primordial electromagnetic fields, Phys. Rev. D 100 (2019) 023524 [arXiv: 1903.02561] [InSPIRE].

[107] L.H. Ford and R.P. Woodard, Stress tensor correlators in the Schwinger-Keldysh formalism, Class. Quant. Grav. 22 (2005) 1637 [gr-qc/0411003] [INSPIRE].

[108] D. Glavan, T. Prokopec and V. Prymidis, Backreaction of a massless minimally coupled scalar field from inflationary quantum fluctuations, Phys. Rev. D 89 (2014) 024024 [arXiv: 1308.5954] [INSPIRE].

[109] D. Glavan, T. Prokopec and D.C. van der Woude, Late-time quantum backreaction from inflationary fluctuations of a nonminimally coupled massless scalar,

Phys. Rev. D 91 (2015) 024014 [arXiv:1408.4705] [INSPIRE].

[110] D. Glavan, T. Prokopec and T. Takahashi, Late-time quantum backreaction of a very light nonminimally coupled scalar, Phys. Rev. D 94 (2016) 084053 [arXiv:1512.05329] [INSPIRE].

[111] D. Glavan, T. Prokopec and A.A. Starobinsky, Stochastic dark energy from inflationary quantum fluctuations, Eur. Phys. J. C 78 (2018) 371 [arXiv:1710.07824] [INSPIRE].

[112] S.P. Miao, T. Prokopec and R.P. Woodard, Deducing cosmological observables from the S-matrix, Phys. Rev. D 96 (2017) 104029 [arXiv:1708.06239] [INSPIRE].

[113] S.P. Miao, T. Prokopec and R.P. Woodard, Scalar enhancement of the photon electric field by the tail of the graviton propagator, Phys. Rev. D 98 (2018) 025022 [arXiv:1806.00742] [INSPIRE].

[114] F. Bezrukov, The Higgs field as an inflaton, Class. Quant. Grav. 30 (2013) 214001 [arXiv: 1307.0708] [INSPIRE].

[115] B. Himmetoglu, C.R. Contaldi and M. Peloso, Instability of anisotropic cosmological solutions supported by vector fields, Phys. Rev. Lett. 102 (2009) 111301 [arXiv:0809.2779] [INSPIRE].

[116] B. Himmetoglu, C.R. Contaldi and M. Peloso, Instability of the ACW model, and problems with massive vectors during inflation, Phys. Rev. D 79 (2009) 063517 [arXiv:0812.1231] [INSPIRE].

[117] G. Esposito-Farese, C. Pitrou and J.-P. Uzan, Vector theories in cosmology, Phys. Rev. D 81 (2010) 063519 [arXiv:0912.0481] [InSPIRE].

[118] M. Karciauskas and D.H. Lyth, On the health of a vector field with $\left(R A^{2}\right) / 6$ coupling to gravity, JCAP 11 (2010) 023 [arXiv: 1007.1426] [INSPIRE].

[119] N.C. Tsamis and R.P. Woodard, General plane wave mode functions for scalar-driven cosmology, Class. Quant. Grav. 21 (2003) 93 [astro-ph/0306602] [inSPIRE].

[120] F.W.J. Olver, D.W. Lozier, R.F. Boisvert and C.W. Clark eds., NIST handbook of mathematical functions, Cambridge University Press, Cambridge, U.K. (2010).

[121] F.W.J. Olver et al. eds., NIST digital library of mathematical functions, release 1.0.23, http://dlmf.nist.gov/, 15 June 2019. 